\title{
EFFECTS OF DISSOLVED GAS SUPERSATURATION ON FISH RESIDING IN THE SNAKE AND COLUMBIA RIVERS, 1996
}

ANNUAL REPORT 1996

\author{
by \\ Boyd P. Schrank \\ Brad a. Ryan \\ and \\ Earl M. Dawley \\ Research Funded by \\ Bonneville Power Administration \\ P.O. Box 3621 \\ Portland, Oregon 97208-362 1 \\ Contract Number 96-AI-93605 \\ Project Number 96-022 \\ and \\ Coastal Zone and Estuarine Studies Division \\ Northwest Fisheries Science Center \\ National Oceanic and Atmospheric Administration \\ 2725 Montlake Boulevard East \\ Seattle, Washington 98 112-2097
}

March 1998 


\section{EXECUTIVE SUMMARY}

Increased spill at dams has commonly brought dissolved gas supersaturation higher than levels established by state and federal water quality criteria in the Columbia and Snake Rivers. These increased spill volumes are intended to provide safe passage for migrating juvenile salmon. However, dissolved gas supersaturation resulting from spill in past decades has led to gas bubble disease (GBD) in fish. Therefore, during the period of high spill in 1996, we monitored the prevalence and severity of gas bubble disease by sampling resident fish in Priest Rapids Reservoir and downstream from Bonneville, Priest Rapids, and Ice Harbor Dams.

We made non-lethal visual examinations of fish using 2.5- to 5-power magnification lenses to assess external signs of GBD (subcutaneous emphysema on fins, head, eyes, and body surface) Subsamples of 5 to 10 resident fish from each sampling day were examined more closely with 20power magnification for gas bubbles in the lateral line, brachial arteries, and gill lamellae.

Subsamples of resident nonsalmonid fish species were held in pens for 4 days and then examined for prevalence and severity of GBD. Three types of pens were used: surface cages held at a depth of 0 to $0.5 \mathrm{~m}$, deep submerged cages held at a depth of 2 to $3 \mathrm{~m}$, and large net-pens with a sloping bottom that extended from the surface to a depth of $4 \mathrm{~m}$.

\section{Gas Bubble Disease Signs in Resident Fish}

Between 15 March and 17 August, we examined 1,172 salmonid fishes, 1,227 nonsalmonid fry, and 9,905 non-salmonid fishes for signs of GBD. Signs of GBD in fish were prevalent downstream from Ice Harbor Dam and in Priest Rapids Reservoir. In other reaches, 
downstream from Bonneville and Priest Rapids Dams, signs of GBD were less prevalent.

State and federal water quality criteria set total dissolved gas saturation (TDGS) of $110 \%$ as the maximum acceptable level. From 1 to $15 \mathrm{~km}$ downstream from Bonneville Dam, TDGS reached $139.9 \%$, and daily average TDGS remained above $125 \%$ from 30 May to 20 June. Spill as high as $259,600 \mathrm{ft}^{3} / \mathrm{sec}$ and $64.5 \%$ of total river flow occurred. On 13 June, prevalence of GBD signs among individual daily fish samples reached 15.8\%. Signs of GBD were observed in $143 \%$ of all fry sampled downstream from Bonneville Dam.

From 15 to $47 \mathrm{~km}$ downstream from Priest Rapids Dam (Hanford Reach), TDGS reached $130 \%$, and daily averages remained above $120 \%$ from 24 May to $21 \mathrm{July}$. Spill as high as $132,500 \mathrm{ft}^{3} / \mathrm{sec}$ and $508 \%$ of total river flow occurred, though prevalence of GBD signs among daily samples never exceeded $15 \%$.

In Priest Rapids Reservoir (downstream from Wanapum Dam), TDGS reached 136\% and daily averages remained above $125 \%$ from 27 May to 24 June as a result of freshet flow past Wanapum Dam. Spill as high as $131,600 \mathrm{ft}^{3} / \mathrm{sec}$ and $50.1 \%$ of total river flow occurred. Prevalence of GBD signs among individual daily fish samples reached $23.1 \%$ on 27 May and $167 \%$ on 3 June.

From 1.6 to $13.7 \mathrm{~km}$ downstream from Ice Harbor Dam, TDGS reached 142\%, and daily averages almost always exceeded $130 \%$ from 1 April to 30 April and from 15 May to 24 June as a result of freshet flows and turbine outages at Ice Harbor Dam Because of high flow and limited turbine capacity, spill as high as $116,900 \mathrm{ft}^{3} / \mathrm{sec}$ and $60.9 \%$ of total river flow occurred Prevalence of GBD signs within individual daily fish samples was greater than $30 \%$ on several occasions (30 May, 6 June, 11 June, and 20 June). 


\section{Gas Bubble Disease in Captive Fish}

Resident nonsalmonid fish used for the net-pen studies were taken from the river and often had signs of GBD at introduction to the pens. After 4 days of holding, GBD signs among the captive fish usually persisted and generally showed an increase in prevalence.

Downstream from Bonneville Dam, fish held in the 0- to 4-m pen showed external GBD signs in 7 of the 13 holding periods; prevalence of external GBD signs ranged from 0 to $58.4 \%$. Prevalence of external GBD signs increased during every 4-day holding period between 17 May and 24 June. When prevalence of external GBD signs increased, mortality ranged from 0 to $4 \%$.

Upstream from Priest Rapids Dam, fish held in the 0- to 4-m pen showed increases of external GBD signs in 15 of the 16 holding periods; prevalence of external GBD signs ranged from 0 to $700 \%$ When prevalence of external GBD signs increased, mortality ranged from 0 to $33 \%$

Downstream from Ice Harbor Dam, fish held in the 0- to 4-m pen showed increases of external GBD signs in 9 of the 13 holding periods; prevalence of external signs ranged from 0 to $860 \%$ When prevalence of external signs of GBD increased, mortality ranged from 4 to $33 \%$.

\section{Model of Gas Bubble Disease Impacts}

In general, we observed high prevalence of GBD signs in fish collected within the sample areas when average daily TDGS exceeded $120 \%$. When TDGS dropped below $120 \%$, we observed low prevalence of GBD signs in sampled fish and low mortalities in captive fish.

Our goal was to provide fishery managers with a definition of TDGS impacts to resident fish throughout affected areas in the Columbia and Snake Rivers. We used sampling and previous 
research data to develop a model providing an estimation of GBD impacts. For the model we attempted to use all data from current and previous study years to predict mortality of resident fish resulting from high TDGS. Unfortunately, mortality in resident fish populations could not be properly evaluated through sampling because dead fish can rarely be recovered from the river. Thus, it was necessary to use captive fish to assess mortality Our first step in developing the model was to analyze the relationship between external GBD signs and TDGS exposure in resident fish. The second step was based on our holding experiments, where we examined the relationship between external GBD signs and mortality.

A mathematical equivalence for increasing, static, and decreasing exposure to TDGS from the Columbia River Operations Hydro-met System was used to develop an exposure index (EI) The EI was correlated with external signs of GBD among resident fish. Correlation was assessed using the following equation for mathematical equivalence and second-order polynomial regression. \%GBD signs $\left.=0.05(\mathrm{EI})^{2} \times 0.2 \mathrm{l}(\mathrm{EI})+0.621\right] \mathrm{R}^{2}=0.79$. Unfortunately our ability to predict mortality was poor, since there was no clear correlation between external GBD signs and mortality in captive fish when data from all species were combined. Data from three resident species (smallmouth bass, yellow perch, and peamouth) produced a stronger correlation; however these relationships were not statistically significant.

\section{Recommendation}

Sampling and holding experiments should be continued in river reaches where TDGS exceeds $120 \%$ To supplement data in the mortality model, the efforts should focus on three species smallmouth bass, yellow perch, and peamouth. 


\section{CONTENTS}

EXECUTIVE SUMMARY $\ldots \ldots \ldots \ldots \ldots \ldots \ldots \ldots \ldots \ldots \ldots$ iii

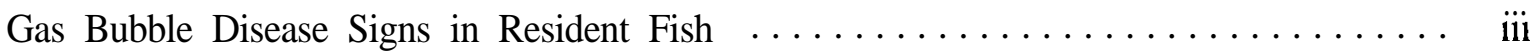

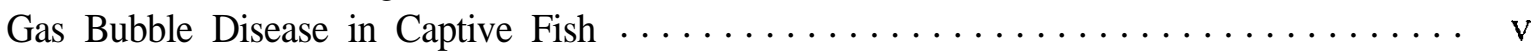

Model of Gas Bubble Disease Impacts $\ldots \ldots \ldots \ldots \ldots \ldots \ldots \ldots \ldots \ldots \ldots \ldots$

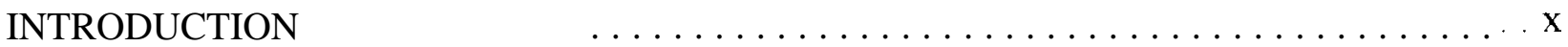

M E T H O D S

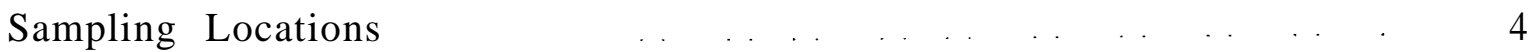

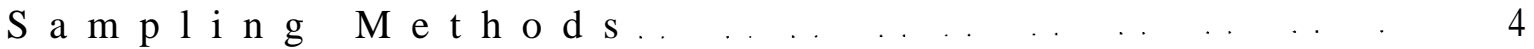

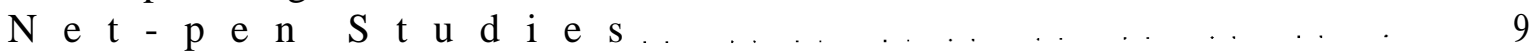

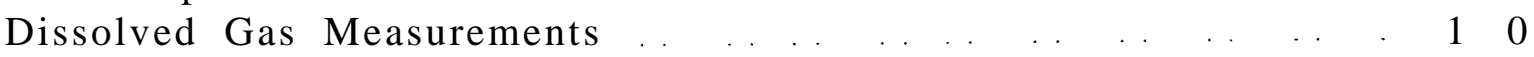

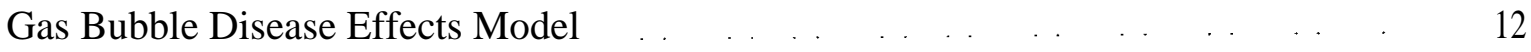

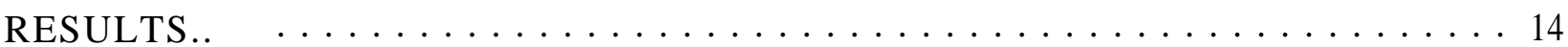

Signs of Gas Bubble Disease in Resident Fish . . . . . . . . . . . . . 14

Downstream from Bonneville Dam . . . . . . . . . . . . . . . 14

Downstream from Priest Rapids Dam $\ldots \ldots \ldots \ldots \ldots \ldots \ldots \ldots \ldots$

Priest Rapids Reservoir … . . . . . . . . . . . . . . . . . . . 22

Downstream from Ice Harbor Dam $\ldots \ldots \ldots \ldots \ldots \ldots \ldots \ldots \ldots$

Gas Bubble Disease in Captive Fish Groups $\ldots \ldots \ldots \ldots \ldots \ldots \ldots \ldots$

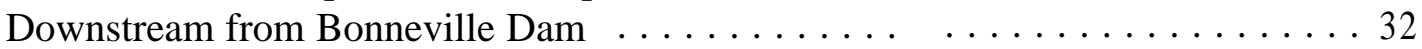

Priest Rapids Reservoir $\ldots \ldots \ldots \ldots \ldots \ldots \ldots \ldots \ldots$

Downstream from Ice Harbor Dam $\ldots \ldots \ldots \ldots \ldots \ldots \ldots \ldots \ldots . \ldots \ldots$

Gas Bubble Disease Effects Model $\ldots \ldots \ldots \ldots \ldots \ldots \ldots \ldots \ldots \ldots$

Exposure vs. Gas Bubble Disease Signs . . . . . . . . . . . . . . . 45

Gas Bubble Disease Signs vs. Mortality . . . . . . . . . . . . . . . . . . 49

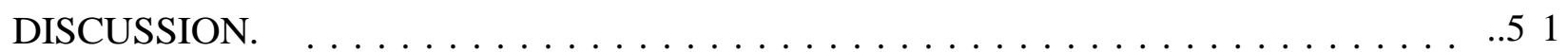

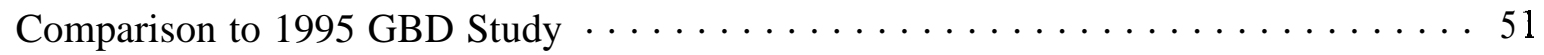

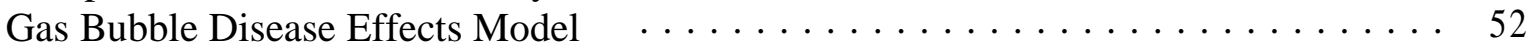

$\begin{array}{llll}\text { CONCLUSIONS } & \text { AND } & \text { RECOMMENDATIONS }\end{array}$

$\begin{array}{lll}\text { ACKNOWLEDGEMENTS } & 5 & 4\end{array}$

$\begin{array}{lll}\text { REFERENCES } & 5 & 5\end{array}$

$\begin{array}{lll}\text { A P P E N D I C E S } & 5 & 8\end{array}$ 


\section{INTRODUCTION}

In recent years, spill has been used to increase survival of juvenile salmonids (Oncorhynchus spp.) passing through Columbia and Snake River dams. Many studies have concluded that spill provides the safest route for juvenile salmonids passing dams on the Columbia and Snake Rivers. However, increased use of spill has raised concern that the resulting increase in dissolved gas levels of the water may be detrimental to aquatic biota. Supersaturation of dissolved atmospheric gases can lead to gas bubble disease (GBD), which is potentially lethal to fish and invertebrates.

During the 1996 spring freshet, dissolved gas levels in the Columbia and Snake Rivers often exceeded $110 \%$ of saturation, the maximum level permitted by the US Environmental Protection Agency, Washington State Department of Ecology, Idaho Department of Environmental Quality, and Oregon State Department of Environmental Quality. The highest levels of supersaturation during this period resulted from conditions over which there was no control, such as high springtime river flows combined with turbine outages at some dams. However, some supersaturation occurred as a result purposeful spill for enhanced fish passage.

In 1994, 1995, and 1996, the U.S. Army Corps of Engineers (COE) obtained a temporary variance for the $110 \%$ saturation maximum standard from the Washington State Department of Ecology and Oregon State Department of Environmental Quality to accommodate spillway passage ofjuvenile salmon. Dissolved gas levels in tailraces at most dams on the lower Snake and Columbia Rivers were allowed to reach $120 \%$ of saturation. An intensified GBD monitoring program was instituted for juvenile salmonids at the dams to evaluate the consequences of this action 
Many studies on GBD and its effect on salmonids have been conducted. From 1968 to 1975, GBD in high-flow years contributed to high mortalities of juvenile salmonids migrating from the Snake River (Ebel et al. 1975). The severity of GBD was dependent upon species, life stage, body size, level of total dissolved gas, duration of exposure, water temperature, general physical condition of the fish, and swimming depth (Ebel et al 1975). Thorough reviews of the literature on dissolved gas supersaturation and of recorded cases of GBD were compiled by Weitkamp and Katz (1980) and updated by Fidler and Miller (1993) Despite numerous studies, there are still questions regarding the total dissolved gas saturation (TDGS) that salmonids can safely tolerate under natural conditions.

When it first became apparent that dissolved gas supersaturation of river water was due to spill at dams and that it caused serious problems for juvenile and adult fish in the Columbia and Snake Rivers, the COE devised methods to reduce dissolved gas supersaturation (Ebel et al. 1975). The methods investigated and implemented were 1) to increase headwater storage to control flow during the spring freshet, 2) to install additional turbines, and 3) to install flow deflectors ("flip-lips") below spillbays to reduce air entrainment in spilled water. As a result of these remedial measures, there was little evidence of GBD in salmonids in the late 1970s and 1980s (Dawley 1986). However, as increased turbine capacity at dams helped reduce TDGS by allowing more river volume to pass through the powerhouse, it also increased the proportion of juvenile salmonids passing dams via turbines. Thus, passage survival at dams was decreased because survival for turbine passage is less than for spillway passage (Schoeneman 1961 )

To improve survival of downstream migrating juvenile salmonids, the present program of 
increased spill was implemented in the 1980s. This spill program resulted in diurnal fluctuations of dissolved gas levels, and in 1985 and 1986 signs of GBD were observed in juvenile and adult salmonids in the Columbia River at McNary, John Day, The Dalles, and Bonneville Dams (Dawley 1986) However, based on low prevalence of GBD signs, it appeared that impacts of dissolved gas supersaturation were minimal, probably because of the short duration of high supersaturation levels In addition, these high levels of dissolved gas resulted from flows exceeding hydro-capacity, not from purposeful spill for enhanced fish survival.

The effects of dissolved gas supersaturation on aquatic biota other than salmonids are not fully understood. Most research has focused on trout and salmon (Weitkamp and Katz 1980), and studies that focused on the occurrence of GBD in resident fish in situ (Dell et al. 1974) were conducted before the implementation of the current spill regime, with its resulting diurnal fluctuations These earlier studies were also conducted before the availability of meters, which allow continuous recording of dissolved gas saturation levels.

The objectives of this study were to assess impacts of ambient levels of gas supersaturated water on fish residing in the Columbia and Snake Rivers and to develop a model that can be used in "real time" by fisheries managers to predict mortality of resident fish resulting from dissolved gas supersaturation 


\section{METHODS}

\section{Sampling Locations}

Sampling in 1996 to assess impacts of GBD in resident fish species was conducted in the lower Columbia River downstream from Bonneville Dam, in the mid-Columbia River downstream and upstream from Priest Rapids Dam, and in the lower Snake River downstream from Ice Harbor Dam. Sampling downstream from Bonneville Dam, River Kilometer (RKm) 218.8 to RKm 229.1, was conducted from 15 March to 12 August (Fig. 1). In the mid-Columbia River, sampling was conducted 15 to $47 \mathrm{~km}$ downstream from Priest Rapids Dam (Hanford Reach), from 10 April to 8 August (Fig. 2) and in Priest Rapids Reservoir from 9 April to 12 August

(Fig 3). In the lower Snake River, sampling was conducted 1.6 to $13.7 \mathrm{~km}$ downstream from Ice Harbor Dam from 16 April to 15 August (Fig. 4).

\section{Sampling Methods}

Resident fish species were collected weekly from each river reach. Electrofishing from a boat equipped with a pair of adjustable booms fitted with umbrella anode arrays was the primary means of fish collection. All electrofishing used pulsed direct current at 30 pulses/second, 400500 volts, and 1-2 amperes. A 7.5-m 2-stick seine with 12.7-mm webbing was also used in some shallow areas (less than $1 \mathrm{~m}$ deep), with two people pulling the seine upstream along the beach.

Downstream from Bonneville Dam, along shorelines having steep gradient, a 3.4-m-deep, 50-m variable-mesh beach seine was used to collect fish. The beach seine consisted of a 14.0-m panel of 19.0-mm mesh, a 17.1 -m panel of 12.7-mm mesh, a 5.5-m panel of 9.5-mm mesh, and a 13.4-m panel of 19.0-mm mesh (all webbing sizes were stretch measure). For deployment, one 


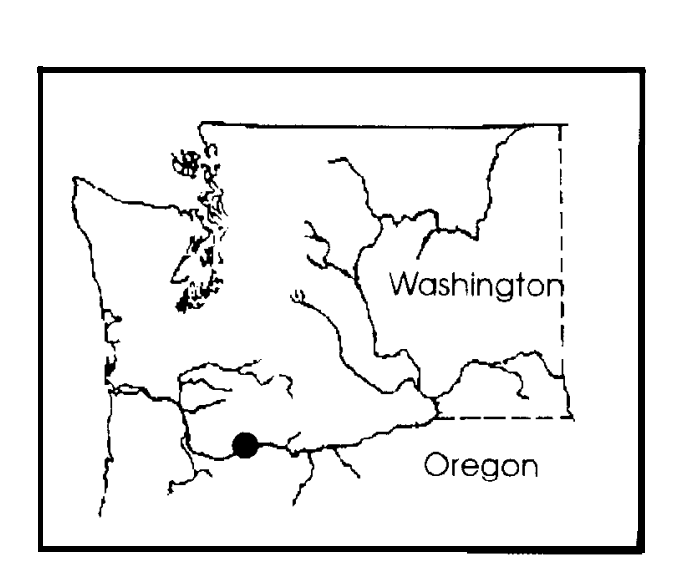

\section{Washington}

\section{Bonneville Dam}

(RKm 234)

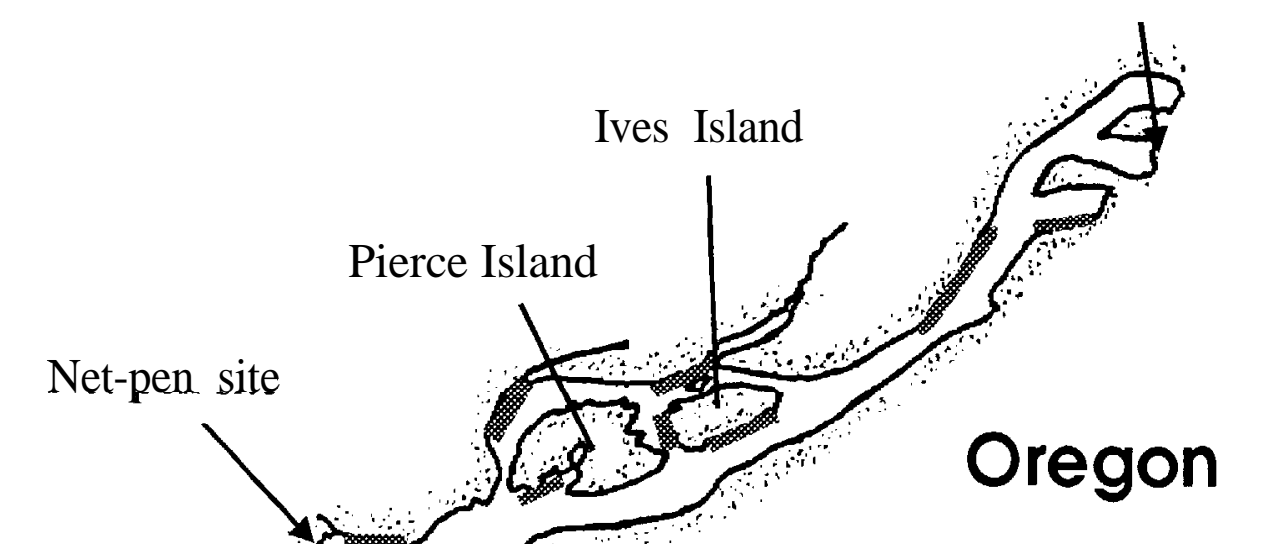

Skamania Island

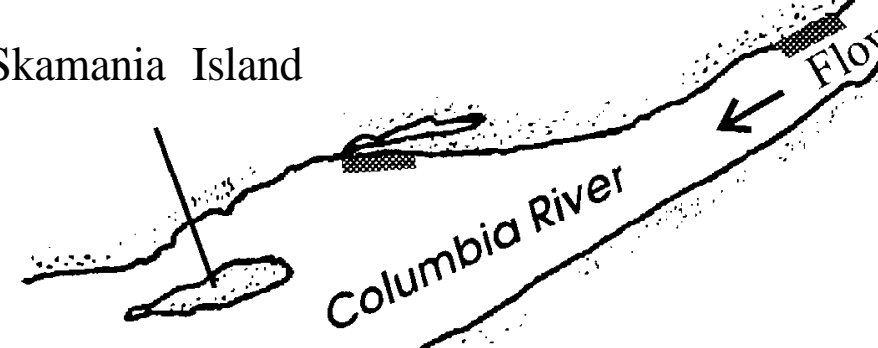

Electrofishing site

Kilometers
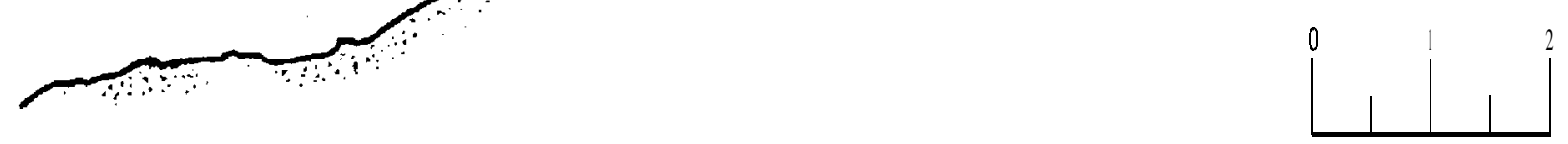

Figure 1. Sampling sites downstream from Bonneville Dam, 1996 


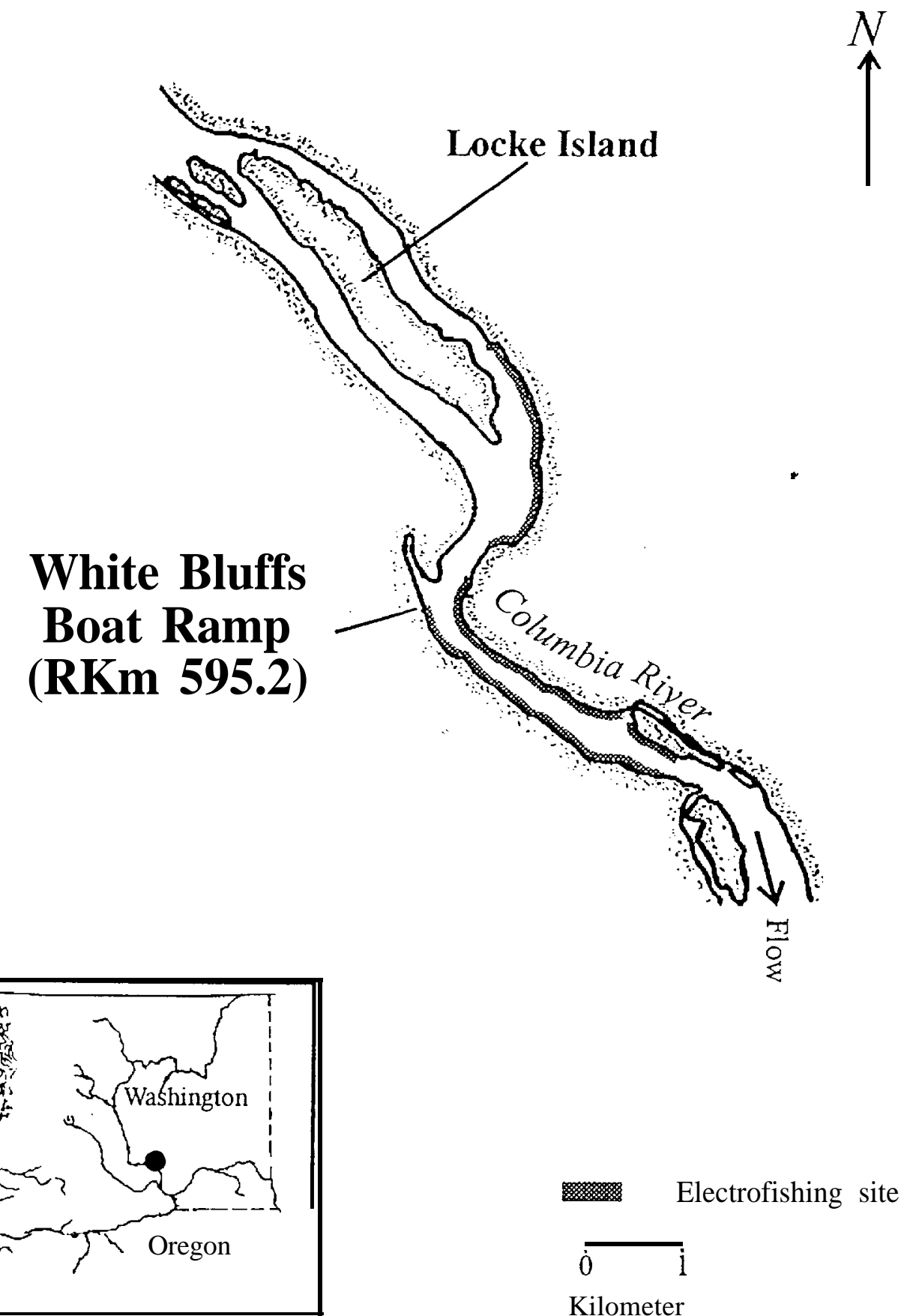

Figure 2. Sampling sites downstream from Priest Rapids Dam, 1996 

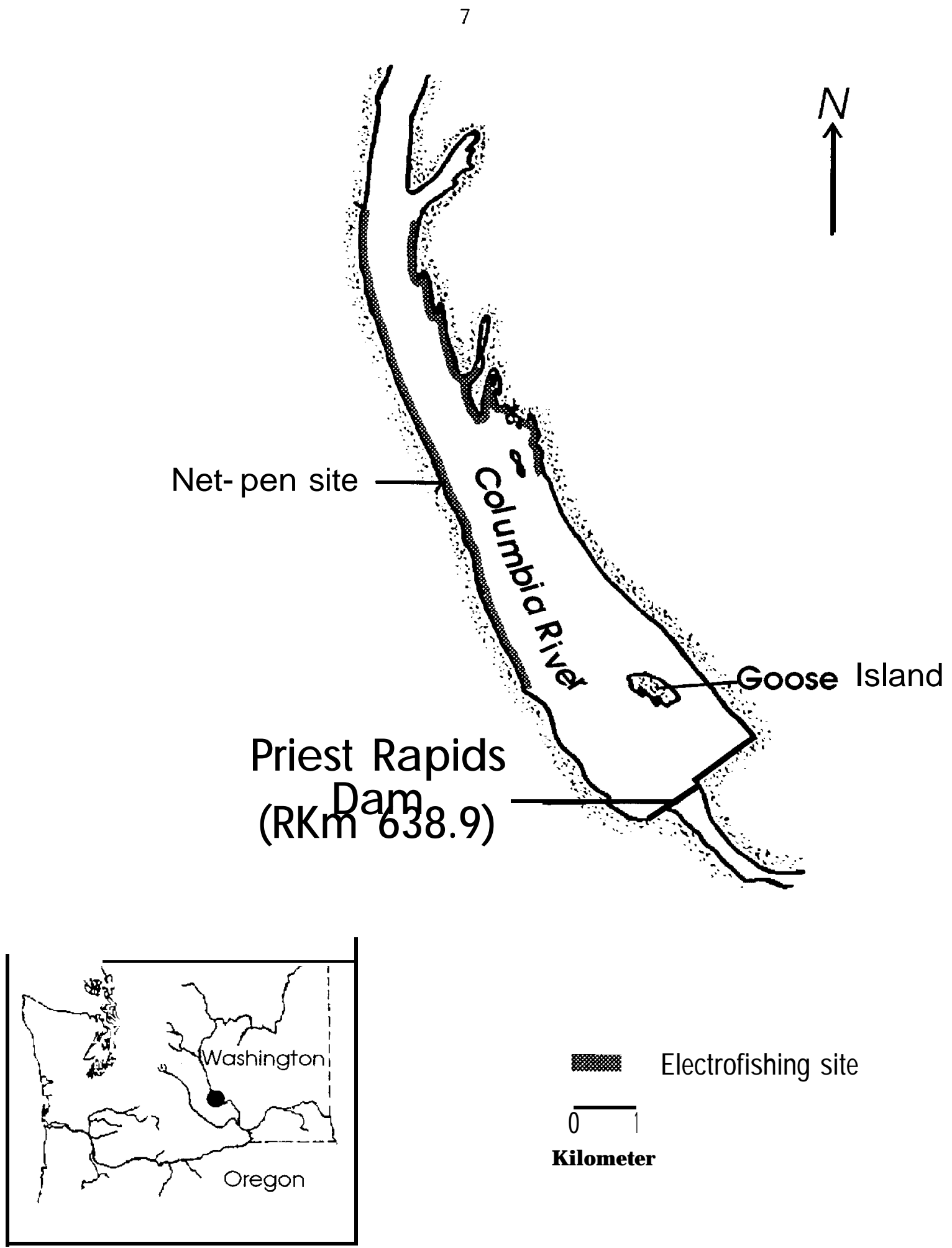

Figure 3. Sampling sites in Priest Rapids Reservoir, 1996 


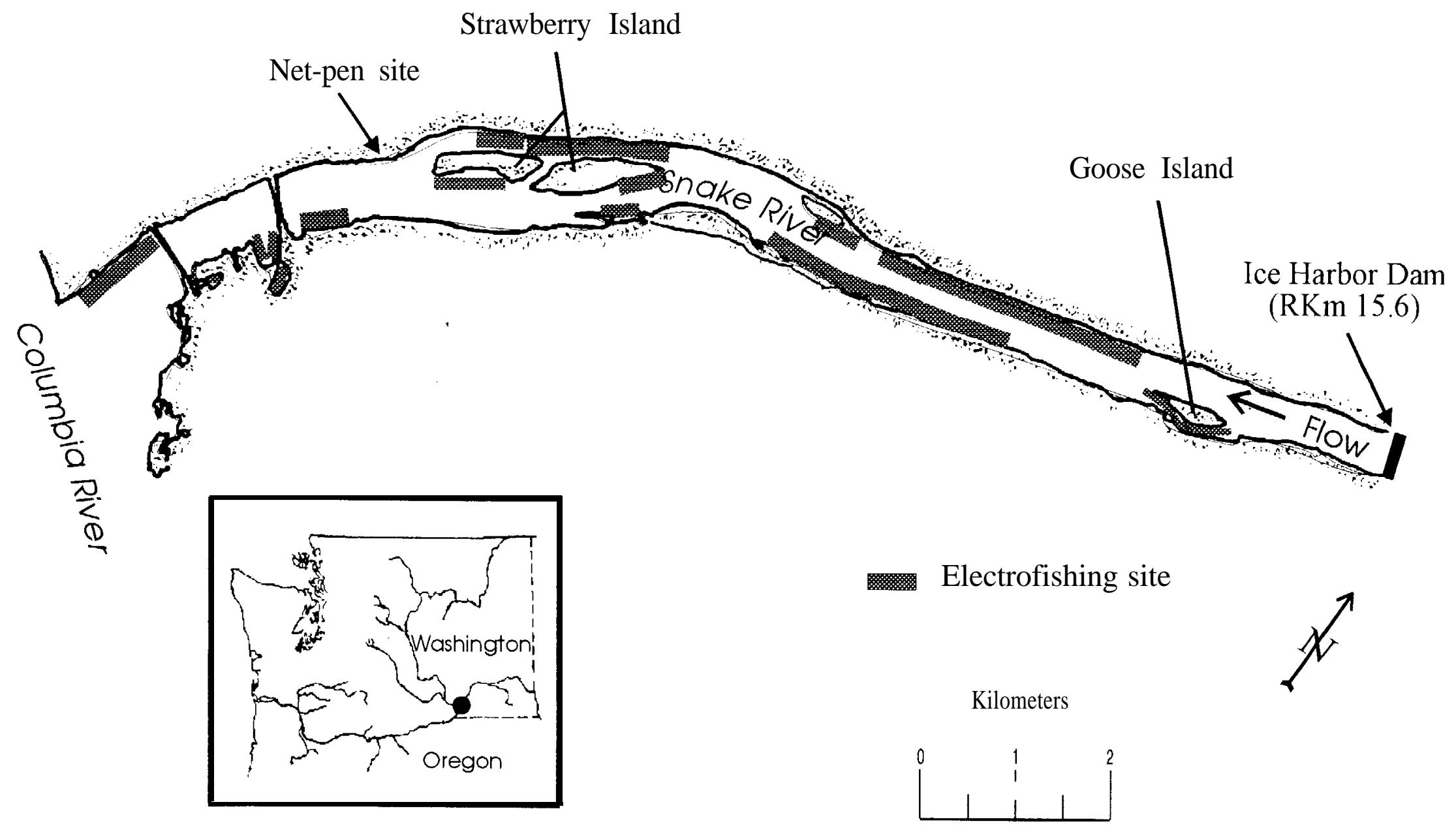

Figure 4. Sampling sites downstream from Ice Harbor Dam, 1996. 
end of the seine was anchored on shore and the other was swung upstream in a wide arc using a S-m outboard-powered boat. The seine was pulled onto the beach by hand, crowding captured

fish into the bunt A small hand-held aquarium net was also used to sample resident fry from near the water surface.

All captured fish were anesthetized using tricaine methane sulfonate (MS-222) identified, measured to the nearest millimeter, and examined for external injuries and signs of GBD (subcutaneous emphysema on fins, head, eyes, and body surface). Individual fish were examined externally using a 2.5 to 5-power headband magnifying lens. Internal examinations of fish were not conducted. Most examinations were made at sampling sites within 15 minutes of collection During examinations, fish were held at ambient temperature and dissolved gas levels. All specimens were allowed to recover fully from the anesthetic prior to release or introduction into holding pens

\section{Net-pen Studies}

Weekly observations of survival rates and changes in prevalence of GBD were made for resident nonsalmonid fish species. Specimens were collected from each river reach, examined for prevalence of GBD, held in enclosures for 4 days, and then reexamined for prevalence of GBD.

Three types of enclosures were used shallow cages held at the surface, which provided a maximum depth of $0.5 \mathrm{~m}(0.6 \times 06 \times 1.0 \mathrm{~m}$ made of perforated aluminum-plate); deep submerged cages held from 20 to $3.0 \mathrm{~m}$ in depth $(0.6 \times 06 \times 10 \mathrm{~m}$ made of perforated aluminum-plate), and large net-pens $(1.8 \times 244 \mathrm{~m})$ with an inclined bottom that extended from the surface to $4 \mathrm{~m}$. 
Built into each net-pen was a webbing partition extending from the water surface to the bottom and running the entire length of the pen (Fig. 5). To help reduce intra-pen predation, fish over $140 \mathrm{~mm}$ were placed on one side of the partition, while resident fish under $140 \mathrm{~mm}$ were placed on the other side. Fish held in net-pens had access from the water surface to a depth of $4 \mathrm{~m}$. Up to 100 individuals of each species were held in these pens. After 4 days, all fish from each of the three enclosure types were reexamined for external signs of GBD and other marks or injuries. Subsamples of up to 10 resident fish were examined more closely for gas bubbles in the lateral line, brachial arteries, and gill lamellae using a dissecting microscope with 20-power magnification. All resident fish mortalities were dissected and examined internally for signs of GBD except those in moderate to extreme states of decomposition.

\section{Dissolved Gas Measurements}

Tensionometers (D'Aoust et al. 1976) were used to measure TDGS at the time and place of sampling fish. Means and ranges of TDGS during 4-day holding periods were determined from dissolved gas data accessed from the Columbia River Operations Hydro-met System (CROHMS) data network of the U.S. Army Corps of Engineers. Additional TDGS data records were obtained every 4 hours at holding locations using tensionometers with data-logging capacity. 

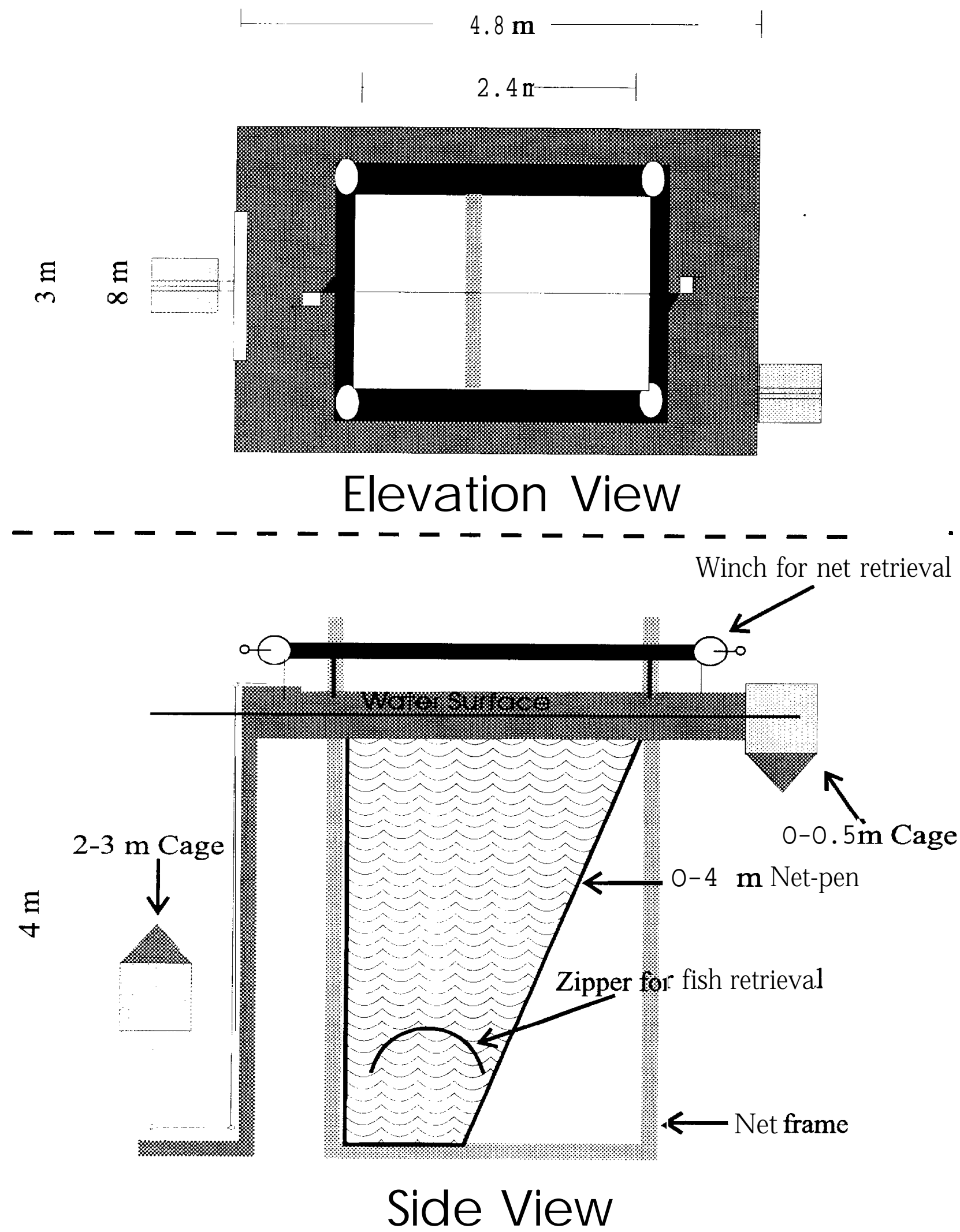

Figure 5. Net-pen, cages, and support barge used for resident fish holding experiments, 1996. 


\section{Gas Bubble Disease Effects Model}

Our GBD effects model associates dissolved gas supersaturation (related to water flow and spill volumes) with the effects of GBD on resident fish in the Columbia River Basin. Using regression analysis, we examined correlations between exposure to ambient TDGS and external signs of GBD on sampled fish and then between percent external GBD signs and percent mortality in fish from the 0- to 4-m-deep net-pen experiments. Data necessary to predict external GBD signs (based on TDGS exposures) and mortality (based on external GBD signs) was obtained from numerous observations $(13,642)$ of feral fish from river sampling and captive fish from netpen experiments. These data included diverse dissolved gas levels observed from 1994 through 1996 in the three river reaches. Modeled effects are not representative of river areas where dissolved gas levels are 7\% lower than measurements of in-river monitors (CROHMS Data) or of fish inhabiting water at depths greater than $3 \mathrm{~m}$.

For analysis of GBD signs in feral fish, a minimum daily sample of at least 50 fish was established to eliminate possible anomalies due to small sample size. We used data only from resident fish sampled in areas where total dissolved gas saturation was within $7 \%$ of the CROHMS 24-hour mean midriver saturation level. This selection was intended to exclude GBD observations from fish inhabiting river locations where total dissolved gas saturations may have differed from those at monitoring stations (back-water ponds and channels).

We focused our sampling efforts for resident fish to depths between 0- and 3-m because the pressure compensation at the 3-m depth is approximately $30 \%$ Therefore, a fish captured at 3-m would not experience effects from dissolved gas supersaturation until TDGS at the surface exceeded 130\% Except salmonids, all captured species that fell within these selection criteria 
were included in the model Fish fry were captured near the surface, and due to early life history stage were highly susceptible to GBD. For these reasons, fry were modeled separately.

Sampling and net-pen data were utilized for our model when collected from a location having continuity of dissolved gas measurements. To utilize sampling data we required a dissolved gas reading every 6 hours for 7 consecutive days prior to and during the sampling activity. To utilize net-pen data we required a total dissolved gas saturation reading every 6 hours during the course of the holding experiment. These criteria eliminated most of our 1994 sampling data because of inconsistent and inaccurate total dissolved gas saturation measurements. Data from samples taken downstream from Priest Rapids Dam were also eliminated due to lack of a monitoring instrument representative of sampling locations

To help ensure that the mortalities were due to GBD, mortality data from the net-pens were only used from high saturation periods (> 120\%) and when external GBD signs were present on surviving fish. For every surviving fish recovered from a qualifying holding experiment, we recorded the percent of surviving fish with external signs of GBD and the percent mortality for that experiment To eliminate anomalies due to small sample sizes, experiments with fish samples smaller than five were not used when mortality data were being used for individual species models. 


\section{RESULTS}

\section{Signs of Gas Bubble Disease in Resident Fish}

\section{Downstream from Bonneville Dam}

Individuals from 10 of the 25 taxa collected downstream from Bonneville Dam displayed external GBD signs. Included were 739 juvenile salmonids, 4,387 nonsalmonids, and 1,227 unidentified fry Among all fish examined, $54 \%$ of salmonids, $2.1 \%$ of resident nonsalmonids, and $14.3 \%$ of fry exhibited signs of GBD (Tables 1 and 2).

From 30 May to 20 June, spill at Bonneville Dam caused TDGS to reach 139\% downstream from the dam. This period corresponded to the greatest prevalence of GBD in sampled fish. Spill volumes ${ }^{1}$ up to $259,600 \mathrm{ft}^{3} / \mathrm{sec}$ and $64.5 \%$ of total river flow occurred (Appendix Fig. 1), and daily average TDGS remained above 125\%. On 13 June, prevalence of GBD signs within individual daily fish samples reached $15.8 \%$ (Table 3)( Fig. 6). External signs of GBD among unidentified fish fry were observed on 8 of the 16 days during which they were sampled (Table 2) Signs of GBD in the lateral line and gill lamellae among fish sampled are summarized in Table 4.

\section{Downstream from Priest Rapids Dam}

Individuals from 9 of the 15 fish taxa collected downstream from Priest Rapids Dam along the Hanford Reach displayed external GBD signs. Included were 353 juvenile salmonids and 943 nonsalmonids External signs of GBD were observed in $2.8 \%$ of salmonids and $6.5 \%$ of resident non-salmonids examined (Table 5).

\footnotetext{
${ }^{1}$ By convention, English units were used for river flow volumes $\left(1,000 \mathrm{ft}^{3} / \mathrm{s}=28.3 \mathrm{~m}^{3} / \mathrm{s}\right)$
} 
Table 1. Numbers sampled, size range, and prevalence of gas bubble disease (GBD) by species for fish collected downstream from Bonneville Dam. 1996.

\begin{tabular}{|c|c|c|c|c|c|}
\hline \multirow[b]{2}{*}{ Species } & \multirow[b]{2}{*}{ Scientific name } & \multirow{2}{*}{$\begin{array}{r}\text { Sample } \\
(\mathrm{n}) \\
\end{array}$} & \multirow{2}{*}{$\begin{array}{l}\text { Length } \\
\text { range" } \\
(\mathrm{mm})\end{array}$} & \multicolumn{2}{|c|}{ Prevalence of $\mathrm{GBD}^{\mathbf{b}}$} \\
\hline & & & & $(\mathrm{n})$ & $(\%)$ \\
\hline Sucker & Catostomus spp. & 1280 & $50-585$ & 42 & 3.3 \\
\hline Peamouth & Mylocheilus caurinus & 916 & $29-447$ & 10 & 1.1 \\
\hline Stickleback & Gasterosteus aculeatus & 657 & $25-109$ & 3 & 0.5 \\
\hline Chinook salmon & Oncorhynchus tshawytscha & 626 & $45-225$ & 25 & 4 \\
\hline Sculpin & Cottus spp. & 596 & $28-420$ & 20 & 3.4 \\
\hline Northern squawfish & Ptychocheilus oregonensis & 443 & $39-603$ & 15 & 3.4 \\
\hline Smallmouth bass & Micropterus dolomieui & 117 & $37-439$ & 0 & \\
\hline Carp & Cyprinus carpio & 116 & $49-720$ & 0 & \\
\hline Coho salmon & Oncorhynchus kisutch & 105 & $113-175$ & 14 & 13.3 \\
\hline Crappie & Pomoxis spp. & 96 & $34-250$ & 1 & 1 \\
\hline Redside shiner & Richardsonius balteatus & 40 & $47-198$ & 1 & 2.5 \\
\hline Yellow perch & Perca flavescens & 26 & $96-24 \quad 1$ & 0 & \\
\hline Chiselmouth & Acrocheilus alutaceus & 21 & $115-352$ & 0 & \\
\hline Killifish & Fundulusspp. & 19 & $52-100$ & 0 & \\
\hline Walleye & Stizostedion vitreum & 12 & $58-710$ & 0 & \\
\hline Bluegill & Lepomis macrochirus & 10 & $95-131$ & 0 & \\
\hline Whitefish & Prosopium spp. & 10 & $88-444$ & 0 & \\
\hline Largemouth bass & Micropterus salmoides & 9 & $52-164$ & 0 & \\
\hline Pumpkinseed & Lepomis gibbosus & 8 & $95-130$ & 0 & \\
\hline Steelhead & Oncorhynchus mykiss & 8 & $165-210$ & 1 & 12.5 \\
\hline Goldfish & Carassins auratus & 5 & $76-252$ & 0 & \\
\hline Bullhead & Ictalurus spp. & 2 & 151 & 0 & \\
\hline American shad & Alosa sapidissima & 1 & 172 & 0 & \\
\hline Dace & Rhinichthys spp. & 1 & 90 & 0 & \\
\hline Starry flounder & Platichthys stellatus & 1 & 115 & 0 & \\
\hline Unidentified fish & & 1 & 46 & $\underline{0}$ & -- \\
\hline Total salmonids & & 739 & & 40 & 5.4 \\
\hline Total nonsalmonids & & 4387 & & 92 & 2.1 \\
\hline
\end{tabular}

a Total lengths were measured for all species except salmonids, for which fork lengths were measured

b External examination for signs of (GBD using a 2.5- to 5.0-power headband magnifying lens. 
Table 2. Total dissolved gas saturation (TDGS) at sampling location, number of fry collected. and prevalence of gas bubble disease (GBD) among fish fry sampled downstream from Bonneville Dam, 1996.

\begin{tabular}{|c|c|c|c|c|}
\hline \multirow[b]{2}{*}{ Date } & \multirow{2}{*}{$\begin{array}{c}\text { Sample Location } \\
\text { TDGS" }\end{array}$} & \multirow{2}{*}{$\begin{array}{l}\text { Fry } \\
(n)^{\text {b.c }}\end{array}$} & \multicolumn{2}{|c|}{ Prevalence of $\mathrm{GBD}^{\mathrm{d}}$} \\
\hline & & & (n) & $(\%)$ \\
\hline 6 Jun & 123.9 & 5 & 5 & 100.0 \\
\hline $11 \mathrm{Jun}$ & 122.7 & 5 & 4 & 80.0 \\
\hline \multirow[t]{3}{*}{12 Jun } & $927^{e}$ & 31 & 0 & 0 \\
\hline & 120.7 & 9 & 1 & 11.1 \\
\hline & 124.7 & 49 & 34 & 69.4 \\
\hline \multirow[t]{3}{*}{13 Jun } & 1199 & 6 & 5 & 83.3 \\
\hline & 1211 & 29 & 0 & 0 \\
\hline & 1254 & 52 & 36 & 69.2 \\
\hline \multirow[t]{2}{*}{18 Jun } & 118.8 & 85 & 0 & 0 \\
\hline & 129.9 & 73 & 48 & 65.8 \\
\hline \multirow[t]{2}{*}{20 Jun } & 95.7 & 51 & 0 & 0 \\
\hline & 123.0 & 64 & 34 & 53.1 \\
\hline 26 Jun & 120.9 & 53 & 8 & 15.1 \\
\hline 27 Jun & 112.9 & 57 & 0 & 0 \\
\hline \multirow[t]{2}{*}{$3 \mathrm{Jul}$} & 114.1 & 102 & 1 & 1.0 \\
\hline & 114.9 & 50 & 0 & 0 \\
\hline $4 \mathrm{Jul}$ & 110.0 & 31 & 0 & 0 \\
\hline l0 Jul & 113.4 & 63 & 0 & 0 \\
\hline I I Jul & 1149 & 55 & 0 & 0 \\
\hline \multirow[t]{2}{*}{$18 \mathrm{Jul}$} & 110.2 & 6 & 0 & 0 \\
\hline & 1124 & 61 & 0 & 0 \\
\hline \multirow[t]{2}{*}{$24 \mathrm{Jul}$} & 1181 & 98 & 0 & 0 \\
\hline & 121.1 & 70 & 0 & 0 \\
\hline $31 \mathrm{Jul}$ & -- & 65 & 0 & 0 \\
\hline \multirow[t]{2}{*}{8 A A ugg - } & 107.1 & 5 & 0 & - \\
\hline & Total & 1227 & 176 & 14.3 \\
\hline
\end{tabular}

Total dissolved gas saturation at the sampling location.

' Number of fry sampled at a particular location on specified day

"Range of total lengths; 1 I-24 mm

d Number and percentage of try displaying signs of gas bubble disease.

e Low saturation probably due to a reduction of water temperature during night hours at France Lake (shallow inlet adjacent to river channel). 
Table 3 Total dissolved gas saturation (TDGS) at sampling sites. prevalence of external signs of gas bubble disease (GBD) by severity, and total prevalence of GBD among resident fish sampled downstream from Bonnerille Dam. 1996.

\begin{tabular}{|c|c|c|c|c|c|c|c|c|c|}
\hline \multirow[b]{4}{*}{ Date } & \multirow{4}{*}{$\begin{array}{c}\text { Sample } \\
\text { (n) }\end{array}$} & \multicolumn{5}{|c|}{ Prevalence of GBD by severity } & \multirow{4}{*}{$\begin{array}{c}\text { Total } \\
\text { prevalence of } \\
G^{\prime} D^{\mathrm{b}} \\
(\%)\end{array}$} & \multirow{3}{*}{\multicolumn{2}{|c|}{$\begin{array}{c}\% \text { TDGS } \\
\text { at sampling sitc(s) }\end{array}$}} \\
\hline & & \multicolumn{4}{|c|}{ Fins $^{a}$} & \multirow{3}{*}{$\begin{array}{l}\text { Body. } \\
\text { eve. head } \\
(n) \\
\end{array}$} & & & \\
\hline & & \multirow{2}{*}{$\begin{array}{c}\text { Rank } \\
1 \\
(\mathrm{n}) \\
\end{array}$} & \multirow{2}{*}{$\begin{array}{c}\text { Rank } \\
2 \\
(\mathrm{n}) \\
\end{array}$} & \multirow{2}{*}{$\begin{array}{c}\text { Rank } \\
3 \\
(\mathbf{n}) \\
\end{array}$} & \multirow{2}{*}{$\begin{array}{c}\text { Rank } \\
4 \\
(\mathrm{n}) \\
\end{array}$} & & & & \\
\hline & & & & & & & & Avg. & Range \\
\hline $15 \mathrm{Mar}$ & 22 & 0 & 1 & 0 & 0 & 0 & 4.5 & 120 & $116.9-123.3$ \\
\hline 16 Mar & 49) & 0 & 0 & 0 & 0 & 0 & 0.0 & 118 & $116.4-1206$ \\
\hline $17 \mathrm{Mar}$ & 5 & 0 & 0 & 0 & 0 & 0 & 0.0 & 124 & One measurement \\
\hline $18 \mathrm{Mar}$ & 147 & 4 & 2 & 1 & 2 & 3 & 8.1 & 120 & $116.3-124.5$ \\
\hline 19 Mar & 134 & 3 & 1 & 2 & 0 & 5 & 8.2 & 118 & $117.5-1183$ \\
\hline $20 \mathrm{Mar}$ & 124 & 1 & 1 & 0 & 0 & 2 & 3.2 & 117 & $116.3-117.8$ \\
\hline $21 \mathrm{Mar}$ & 109 & 0 & 0 & 0 & 1 & 3 & 3.7 & 119 & $118.4-119.3$ \\
\hline $22 \mathrm{Mar}$ & 126 & 0 & 2 & 0 & 0 & 0 & 1.6 & 118 & $117.5-118.9$ \\
\hline 23 Mar & 18 & 1 & 0 & 0 & 0 & 0 & 5.6 & 117 & One measurement \\
\hline 24 Mar & 135 & 0 & 0 & 0 & 0 & 0 & 0.0 & 112 & One measurement \\
\hline $8 \mathrm{Apr}$ & 27 & 0 & 0 & 0 & 0 & 0 & 0.0 & 120 & One measurement \\
\hline 19 Apr & 52 & 2 & 0 & 0 & 0 & 0 & 3.8 & 117 & One measurement \\
\hline $24 \mathrm{Apr}$ & 11 & 0 & 0 & 0 & 0 & 0 & 0.0 & 119 & One measurement \\
\hline $25 \mathrm{Apr}$ & 113 & 1 & 0 & 0 & 1 & 0 & 1.8 & 119 & $113.3-122.2$ \\
\hline 30 Apr & 66 & 0 & 0 & 0 & 0 & 0 & 0.0 & 124 & $120.3-127.5$ \\
\hline 2 Mas & 21 & 0 & 0 & 0 & 1 & 0 & 4.8 & 116 & One measurement \\
\hline $6 \mathrm{May}$ & 101 & 1 & 0 & 1 & 0 & 0 & 2.0 & 118 & $117.3-118.9$ \\
\hline $10 \mathrm{May}$ & 20 & 0 & 0 & 0 & 0 & 0 & 0.0 & 110 & One measurement \\
\hline $13 \mathrm{Max}$ & 117 & 2 & 0 & 0 & 0 & 0 & 1.7 & 114 & $112.7-114.7$ \\
\hline $16 \mathrm{May}$ & 17 & 0 & 0 & 0 & 0 & 0 & 0.0 & 122 & One measurement \\
\hline $20 \mathrm{Mas}$ & 120 & 1 & 0 & 0 & 0 & 1 & 1.6 & 117 & $112.0-121.8$ \\
\hline $23 \mathrm{May}$ & 28 & 0 & 0 & 0 & 0 & 0 & 0.0 & 119 & One measurement \\
\hline 2XMar & 114 & 5 & 0 & 0 & 0 & 0 & 4.4 & 121 & $118.2-123.8$ \\
\hline 30 Мат & 25 & 0 & 0 & 0 & 0 & 0 & 0.0 & 125 & One measurement \\
\hline 3 Jun & 133 & 15 & 1 & 0 & 0 & 2 & 135 & 126 & $127.0-125.6$ \\
\hline 6 Jun & 37 & 1 & 0 & 0 & 0 & 0 & 2.7 & 124 & Onc measurement \\
\hline IO Jun & 162 & 10 & 1 & 2 & 0 & 1 & 8.6 & 126 & $1242-128.1$ \\
\hline II Jun & 11 & 0 & 0 & 0 & 0 & 0 & 0.0 & 125 & $1227-126.6$ \\
\hline $13 \mathrm{Jun}$ & 38 & 4 & 1 & 0 & 1 & 0 & 158 & 121 & Onc measurement \\
\hline $17 \mathrm{Jun}$ & 160 & 5 & 1 & 0 & 0 & 0 & 3.8 & 122 & $117.8-123.7$ \\
\hline $19 \mathrm{Jun}$ & 29 & 1 & 0 & 0 & 0 & 0 & 3.4 & 127 & One measurement \\
\hline
\end{tabular}


Table 3. Continued

\begin{tabular}{|c|c|c|c|c|c|c|c|c|c|}
\hline \multirow[b]{4}{*}{ Date } & \multirow{4}{*}{$\begin{array}{c}\text { Sample } \\
\text { (n) }\end{array}$} & \multicolumn{5}{|c|}{ Prevalence of GBD by scverity } & \multirow{4}{*}{$\begin{array}{c}\text { Prevalence of } \\
\text { GBD }^{b} \\
(\%)\end{array}$} & \multirow{3}{*}{\multicolumn{2}{|c|}{$\begin{array}{c}\% \text { TDGS } \\
\text { at sampling site(s) }\end{array}$}} \\
\hline & & \multicolumn{4}{|c|}{ Fins" } & \multirow{3}{*}{$\begin{array}{c}\text { Body } \\
\text { eye, head } \\
(n)\end{array}$} & & & \\
\hline & & \multirow{2}{*}{$\begin{array}{c}\text { Rank } \\
1 \\
(\mathrm{n}) \\
\end{array}$} & \multirow{2}{*}{$\begin{array}{c}\text { Rank } \\
2 \\
(\mathrm{n})\end{array}$} & \multirow{2}{*}{$\begin{array}{c}\text { Rank } \\
3 \\
(\mathrm{n}) \\
\end{array}$} & \multirow{2}{*}{$\begin{array}{c}\text { Rank } \\
4 \\
(\mathrm{n}) \\
\end{array}$} & & & & \\
\hline & & & & & & & & Avg. & Range \\
\hline 24 Jun & 110 & 0 & 0 & 0 & 0 & 0 & 0.0 & 119 & $111.0-124.1$ \\
\hline 25 Jun & 30 & 1 & 0 & 0 & 0 & 0 & 3.3 & 117 & One measurement \\
\hline 26 Jun & 73 & 3 & 0 & 0 & 0 & 1 & 5.5 & 124 & $120.9-130.5$ \\
\hline 27 Jun & 134 & 2 & 0 & 0 & 0 & 2 & 3.0 & 119 & $116.3-121.1$ \\
\hline $1 \mathrm{Jul}$ & 124 & 0 & 0 & 0 & 0 & 0 & 00 & 119 & $118.3-119.6$ \\
\hline $2 \mathrm{Jul}$ & 43 & 1 & 0 & 0 & 0 & 0 & 2.3 & 117 & One measurement \\
\hline $3 \mathrm{Jul}$ & 96 & 0 & 0 & 0 & 0 & 0 & 0.0 & 114 & $113.3-114.8$ \\
\hline $4 \mathrm{Jul}$ & 155 & 0 & 0 & 0 & 0 & 0 & 0.0 & 110 & 109. 1-1 10.0 \\
\hline $8 \mathrm{Jul}$ & 123 & 0 & 0 & 0 & 0 & 0 & 0.0 & 119 & $118.6-119.1$ \\
\hline $9 \mathrm{Jul}$ & 49 & 0 & 0 & 0 & 0 & 0 & 0.0 & 114 & $113.4-115.4$ \\
\hline $11 \mathrm{Jul}$ & 198 & 0 & 0 & 0 & 0 & 0 & 0.0 & 109 & $107.6-110.3$ \\
\hline $15 \mathrm{Jul}$ & 167 & 1 & 0 & 0 & 0 & 0 & 0.6 & 119 & $118.3-118.8$ \\
\hline $16 \mathrm{Jul}$ & 74 & 0 & 0 & 0 & 0 & 0 & 0.0 & 113 & One measurement \\
\hline $18 \mathrm{Jul}$ & 130 & 0 & 0 & 0 & 0 & 0 & 0.0 & 110 & $109.2-110.8$ \\
\hline $22 \mathrm{Jul}$ & 166 & 0 & 0 & 0 & 0 & 0 & 0.0 & 116 & $115.3-116.7$ \\
\hline $23 \mathrm{Jul}$ & 37 & 0 & 0 & 0 & 0 & 0 & 0.0 & 117 & One measurement \\
\hline $25 \mathrm{Jul}$ & 166 & 0 & 0 & 0 & 0 & 2 & 1.2 & 117 & $116.4-117.5$ \\
\hline $29 \mathrm{Jul}$ & 243 & 1 & 2 & 0 & 0 & 5 & 3.3 & 112 & $108.0-115.7$ \\
\hline $30 \mathrm{Jul}$ & 134 & 3 & 0 & 0 & 0 & 3 & 4.4 & 111 & One measurement \\
\hline $31 \mathrm{Jul}$ & 266 & 0 & 0 & 0 & 0 & 0 & 00 & 109 & $108.2-109.2$ \\
\hline 5 Aug & 136 & 1 & 0 & 0 & 0 & 1 & 1.4 & 109 & $106.5-111.0$ \\
\hline 7 Aug & 156 & () & 0 & 0 & 0 & 0 & 0.0 & 111 & $109.3-111.5$ \\
\hline 12 Aug & 50 & 0 & 0 & 0 & 0 & 0 & 0.0 & 108 & One measurement \\
\hline
\end{tabular}

a Rank (determined from percent of total fin area affected with emphysema): $1=1-5 \%, 2=6-25 \%, 3=26-50 \% .4=$ $>50 \%$.

${ }^{\mathrm{h}}$ Not including fish with (IBD in lateral line and/or gill. 
\%. Range TDGS (\%)

GBD \%, Sample

40

-Daily average TDGS (\%)

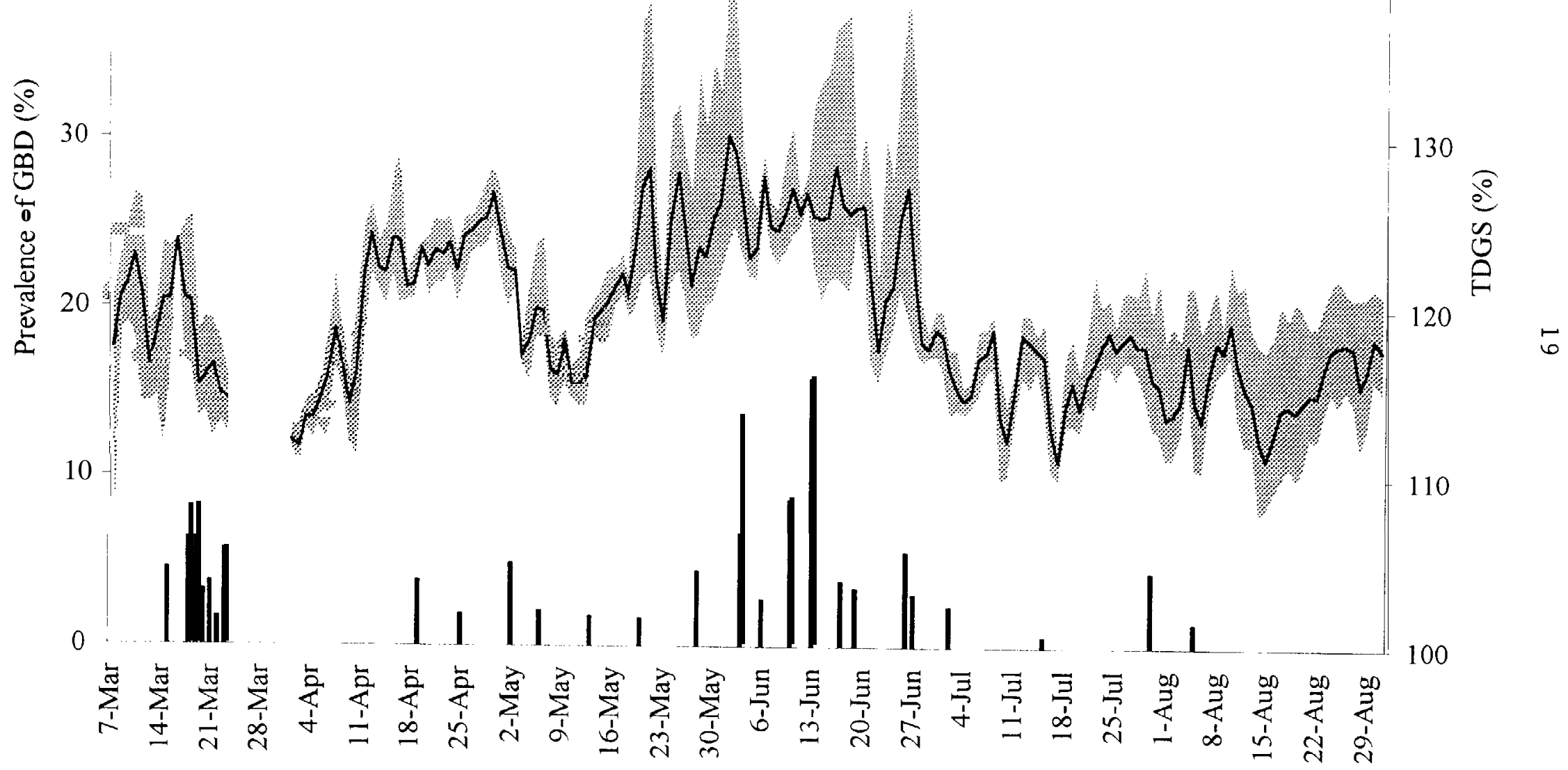

Figure 6. Prevalence of GBD in resident fish collected downstream from Bonneville Dam compared with daily average and range of total dissolved gas saturation (TDGS) (COE, Skamania). 
Table 4. Total dissolved gas saturation (TDGS) at sampling sites and prevalence of gas bubble disease (GBD) signs in the lateral line and gill lamellae among resident fish, 1996.

\begin{tabular}{|c|c|c|c|c|c|c|c|c|c|c|}
\hline \multirow[b]{4}{*}{ Date } & \multicolumn{5}{|c|}{ Downstream from Bonneville Dam } & \multicolumn{5}{|c|}{ Downstream from Priest Rapids Dam } \\
\hline & \multirow{3}{*}{$\begin{array}{c}\text { Lateral } \\
\text { line }\end{array}$} & \multirow{3}{*}{$\begin{array}{c}\text { Gill } \\
\text { lamellae }\end{array}$} & \multicolumn{3}{|c|}{ \% TDGS at sampling site(s) } & \multirow[b]{3}{*}{ Date } & \multirow{3}{*}{$\begin{array}{c}\text { Lateral } \\
\text { line }^{\mathbf{a}}\end{array}$} & \multirow{3}{*}{$\begin{array}{c}\text { Gill } \\
\text { lamellae }\end{array}$} & \multicolumn{2}{|c|}{$\%$ TDGS at sampling site(s) } \\
\hline & & & \multirow[b]{2}{*}{ Avg. } & \multicolumn{2}{|c|}{ Range } & & & & \multirow[b]{2}{*}{ Av $?$} & Range \\
\hline & & & & Min & Max. & & & & & Max. \\
\hline $15 \mathrm{Mar}$ & $1 / 8$ & $1 / 8$ & 120 & 1169 & 123.3 & $18 \mathrm{Apr}$ & $1 / 10$ & $0 / 0$ & 121 & $119.3 \quad 124 \quad 3$ \\
\hline $16 \mathrm{Mat}$ & $1 / 6$ & $(1 / 6$ & 118 & 116.4 & 120.6 & $1 \mathrm{May}$ & $1 / 10$ & $0 / 10$ & 130 & One measurement \\
\hline 17 Mar & $1 / 5$ & $0 / 5$ & 124 & \multicolumn{2}{|c|}{ One measurement } & $2 \mathrm{May}$ & $0 / 10$ & $0 / 10$ & 124 & One measurement \\
\hline $18 \mathrm{Mar}$ & $3 / 6$ & $0 / 6$ & 120 & 1163 & 124.5 & 7 May & $8 / 10$ & $0 / 10$ & 120 & $\begin{array}{lll}1183 & 121 & 2\end{array}$ \\
\hline 1) Mar & $2 / 2$ & $(1 / 2$ & 118 & 117.5 & 118.3 & $16 \mathrm{Mas}$ & $2 / 5$ & $3 / 5$ & 120 & One measurement \\
\hline 20 Mar & $6 / 7$ & $(/ / 7$ & 117 & 116.3 & 117.8 & $22 \mathrm{May}$ & $4 / 5$ & $1 / 5$ & 119 & One measurement \\
\hline $21 \mathrm{Mar}$ & $3 / 15$ & $0 / 15$ & 119 & 118.4 & 119.3 & 23 May & $6 / 10$ & $5 / 10$ & 115 & One measurement \\
\hline $22 \mathrm{Mar}$ & $1 / 7$ & ()$/ 7$ & 118 & 117.5 & 118.9 & 29 May & $2 / 9$ & $1 / 9$ & 128 & One measurement \\
\hline $23 \mathrm{Mar}$ & $2 / 7$ & $(1 / 7$ & 117 & \multicolumn{2}{|c|}{ One measurement } & 5 Jun & $0 / 1$ & $1 / 1$ & 122 & $120.6 \quad 123.3$ \\
\hline $24 \mathrm{Mar}$ & $3 / 7$ & $0 / 7$ & 112 & \multicolumn{2}{|c|}{ One measurement } & 12 Jun & $1 / 10$ & $1 / 10$ & 121 & $120.7 \quad 121.2$ \\
\hline $8 \wedge p r$ & $2 / 10$ & $0 / 0$ & 120 & \multicolumn{2}{|c|}{ ( )ne measurement } & $20 \mathrm{Jun}$ & $0 / 5$ & $1 / 5$ & 125 & One measurement \\
\hline 2 May & $2 / 10$ & $0 / 10$ & 116 & \multicolumn{2}{|c|}{ One measurement } & 3 Jul & $0 / 3$ & $0 / 3$ & 124 & One measurement \\
\hline 10 Mas & $3 / 9$ & $(1 / 9$ & 110 & \multicolumn{2}{|c|}{ One measurement } & $10 \mathrm{Jul}$ & 1/1 1 & $1 / 11$ & 118 & $\begin{array}{lll}116.9 & 1 & 18.7\end{array}$ \\
\hline $16 \mathrm{Ma}$ & $4 / 10$ & $0 / 10$ & 122 & ( ) ne $\mathrm{m}$ & urement & $17 \mathrm{Jul}$ & $1 / 10$ & $2 / 10$ & 121 & 120.4 \\
\hline $23 \mathrm{Mal}$ & $0 / 10$ & $0 / 10$ & 119 & One $\mathrm{m}$ & urement & 8 Aug & $0 / 5$ & $3 / 5$ & 114 & One measurement \\
\hline 30 Mar & $3 / 10$ & ()$/ 10$ & 125 & One $\mathrm{m}$ & arement & & & & & \\
\hline ३ Jum & $1 / 1$ & (1)/1 & 126 & 127.0 & 125.6 & & & & & \\
\hline $6 . J \ln$ & $1 / 10$ & $1 / 10$ & 124 & One $\mathrm{m}$ & arement & & & & & \\
\hline $11 \mathrm{Jun}$ & $2 / 9$ & $0 / 9$ & 125 & 122.7 & 126.6 & & & & & \\
\hline 19. Jum & $2 / 10$ & $0 / 10$ & 127 & One $\mathrm{m}$ & urement & & & & & \\
\hline $25 \mathrm{Jun}$ & $1 / 10$ & $(0 / 10$ & 117 & One $\mathrm{ms}$ & arement & & & & & \\
\hline 2 Jul & $3 / 10$ & $(1 / 10$ & 117 & One $\mathrm{m}$ & urement & & & & & \\
\hline 9.Jul & $0 / 10$ & $(/ / 10$ & 114 & 113.4 & 115.4 & & & & & \\
\hline 16 Jul & $0 / 10$ & $0 / 10$ & 113 & One $\mathrm{m}$ & arement & & & & & \\
\hline $23 . J u l$ & $0 / 10$ & $(/ / 10$ & 117 & One $\mathrm{m}$ & rement & & & & & \\
\hline 30) Jul & $0 / 13$ & $(1 / 13$ & 111 & One $\mathrm{m}$ & arement & & & & & \\
\hline & Instrea & $m$ from Prie & $\underline{\text { st Ran }}$ & s Dam & & & Down & tream from & Ice $\mathrm{Ha}$ & or Dam \\
\hline & & & $\% \mathrm{TD}$ & at sar & ng site (s) & & & & $\% \mathrm{TD}$ & at sampling site(s) \\
\hline & I ateral & Gill & & & & & Lateral & Gill & & Range \\
\hline Date & line" & lamellae & Avg & Min. & Max. & Date & $\operatorname{lin}$ & lamell & Avg. & Max. \\
\hline $17 \mathrm{Apr}$ & $4 / 10$ & $0 / 0$ & 128 & 128.0 & 128.0 & 29 Apr & $1 / \mathrm{h}$ & $1 / 6$ & 129 & 135.3 \\
\hline $30 \mathrm{Apr}$ & $1 / 6$ & $0 / 6$ & 115 & One $\mathrm{m}$ & urement & 6 May & $8 / 11$ & $1 / 11$ & 118 & One measurement \\
\hline $8 \mathrm{May}$ & $9 / 10$ & $2 / 10$ & 126 & One $\mathrm{m}$ & arement & $14 \mathrm{May}$ & $5 / 6$ & $3 / 6$ & 123 & One measurement \\
\hline I 3 May & $8 / 10$ & $4 / 10$ & 123 & One $\mathrm{m}$ & arement & 21 May & $6 / 10$ & $2 / 10$ & 132 & $\begin{array}{lll}125.2 & 137 & 8\end{array}$ \\
\hline $20 \mathrm{May}$ & $8 / 11$ & $5 / 11$ & 131 & One $\mathrm{m}$ & urement & 28 May & $7 / 10$ & $5 / 10$ & 130 & One measurement \\
\hline 27 Mas & $1 / 10$ & $0 / 10$ & 124 & One $\mathrm{m}$ & arement & 30 May & $3 / 7$ & $0 / 7$ & 135 & 131.1 \\
\hline 3.Jun & $6 / 10$ & $1 / 10$ & 133 & One $\mathrm{m}$ & arement & 13 Jun & $2 / 10$ & $0 / 10$ & 123 & One measurement \\
\hline 10 Jun & $2 / 10$ & $4 / 10$ & 130 & One $\mathrm{m}$ & urement & I 9 Jun & $5 / 10$ & $0 / 10$ & 120 & 117.1 \\
\hline 17 Jun & $2 / 10$ & $0 / 10$ & 109 & One $\mathrm{m}$ & urement & 25 Jun & $1 / 8$ & $0 / 8$ & 114 & 113.9 \\
\hline $24 J u n$ & $3 / 11$ & $2 / 1 \mathrm{I}$ & 121 & 1190 & 122.4 & $9 \mathrm{Jul}$ & $4 / 5$ & $0 / 5$ & 116 & $\begin{array}{llll}1 & 10.0 & 121 & 1\end{array}$ \\
\hline $1 \mathrm{Jul}$ & $2 / 9$ & $1 / 10$ & 103 & One $\mathrm{m}$ & urement & $11 \mathrm{Jul}$ & $1 / 4$ & $1 / 4$ & 119 & $\begin{array}{lll}1 & 18.2 & 120.2\end{array}$ \\
\hline $8 . J u l$ & $2 / 10$ & $(1 / 10$ & Il) & ()ne $\mathrm{m}$ & arement & 18Jul & $1 / 6$ & $4 / 6$ & 103 & One measurement \\
\hline $15 \mathrm{Jul}$ & $1 / 8$ & $1 / 8$ & 134 & One $\mathrm{m}$ & urement & 30) Jul & $2 / 5$ & $1 / 5$ & 100 & One measurement \\
\hline 20 Jul & $0 / 6$ & $2 / 6$ & 95 & One $\mathrm{m}$ & arement & 13 Aug & $1 / 2$ & $0 / 2$ & 105 & One measurement \\
\hline
\end{tabular}

aumber of fish with (iHBD signs in the lateral line/number examined.

b Number- of tish with (iBI) signs in gill lamellae/number examined. 
Table 5 Numbers sampled, size range. and prevalence of gas bubble disease (GBD) by species for fish collected downstream from Priest Rapids Dam. 1996.

\begin{tabular}{|c|c|c|c|c|c|}
\hline \multirow[b]{2}{*}{ Species } & \multirow[b]{2}{*}{ Scientific name } & \multirow{2}{*}{$\begin{array}{c}\text { Sample } \\
\text { (n) }\end{array}$} & \multirow{2}{*}{$\begin{array}{l}\text { Length } \\
\text { range }^{\mathrm{a}} \\
(\mathrm{mm})\end{array}$} & \multicolumn{2}{|c|}{ Prevalence of GBD ${ }^{\mathrm{b}}$} \\
\hline & & & & (n) & $(\%)$ \\
\hline Sucker & Catostomusspp & 371 & $52-760$ & 49 & 13.2 \\
\hline Northern squawfish & Ptychocheilus oregonensis & 284 & $60-493$ & 6 & 2.1 \\
\hline Chinook salmon & Oncorhynchus tshawytscha & 231 & $36-195$ & 7 & 3.0 \\
\hline Steelhead & Oncorhynchus mykiss & 95 & $37-254$ & 1 & 1.1 \\
\hline Pcamouth & Mylocheilus caurnus & 91 & $40-480$ & 1 & 1.1 \\
\hline Redside shiner & Richardsonius halteatus & 70 & $45-151$ & 1 & 1.4 \\
\hline Chiselmouth & Acrocheihus alutaceus & 66 & $61-290$ & 3 & 4.5 \\
\hline Sockeye salmon & Oncorhynchus nerka & 27 & $147-232$ & 2 & 7.4 \\
\hline Moutain whitefish & Prosopium williamsoni & 14 & $166-440$ & 0 & 0.0 \\
\hline Smallmouth bass & Micropterus doloment & 13 & $74-250$ & 1 & 77 \\
\hline Carp & Cyprinus carpio & 11 & $84-670$ & 0 & \\
\hline Sculpin & Cottus spp. & 10 & $58-203$ & 0 & \\
\hline Yellow perch & Perca flavescens & 10 & $45-181$ & 0 & \\
\hline Stickleback & Gasterosteus aculeatus & 2 & 59 & 0 & \\
\hline Bullhead & Ictalurus spp. & 1 & 172 & 0 & - \\
\hline Total salmonids & & 353 & & 10 & 2.x \\
\hline Total nonsalmonids & & 943 & & 61 & 6.5 \\
\hline
\end{tabular}

a Total lengths were measured for all species except salmonids. for which fork lengths were measured. 
From 24 May to 21 June, spill at Priest Rapids Dam caused TDGS to reach $130 \%$ downstream from the dam. Spill volumes up to $132,500 \mathrm{ft}^{3} / \mathrm{sec}$ and $50.8 \%$ of total river flow occurred (Appendix Fig. 2), and daily average TDGS remained near 125\%. Prevalence of external GBD signs within individual daily samples never exceeded 15\% (Table 6)(Fig. 7). Signs of GBD in the lateral line and gill lamellae are summarized in Table 4.

\section{Priest Rapids Reservoir}

Individuals from 11 of the 20 taxa collected in Priest Rapids Reservoir displayed external signs of GBD. Included were 60 juvenile salmonids and 2,220 resident nonsalmonids. Among all fish examined, no salmonids and $7.3 \%$ of resident nonsalmonids exhibited external signs of GBD (Table 7)

From 27 May to 24 June, spill at Wanapum Dam caused TDGS to reach $136 \%$ in Priest

Rapids Reservoir This period corresponded to the greatest prevalence of external GBD signs in sampled fish Spill volumes up to $131,600 \mathrm{ft}^{3} / \mathrm{sec}$ and $50.1 \%$ of total river flow occurred (Appendix Fig 3), and daily average TDGS remained above 125\%. Prevalence of GBD signs within individual daily fish samples reached $23.1 \%$ on 27 May and $16.7 \%$ on 3 June (Table 8 ) (Fig. 8). Signs of GBD in the lateral line and gill lamellae among fish sampled are summarized in Table 4 
Table 6 Total dissolved gas saturation (TDGS) at sampling sites. prevalence of estemal signs of gas bubble disease (GBD) by severity, and total prevalence of GBD among resident fish sampled downstream from Priest Rapids Dam. 1996.

\begin{tabular}{|c|c|c|c|c|c|c|c|c|c|}
\hline \multirow[b]{4}{*}{ Date } & \multirow{4}{*}{$\begin{array}{c}\text { Sample } \\
\text { (n) }\end{array}$} & \multicolumn{5}{|c|}{ Prevalence of GBD by severity } & \multirow{4}{*}{$\begin{array}{c}\text { Total } \\
\text { prevalence of } \\
\text { GBD }^{b} \\
(\%) \\
\end{array}$} & \multirow{3}{*}{\multicolumn{2}{|c|}{$\begin{array}{c}\% \text { TDGS } \\
\text { at sampling sites }\end{array}$}} \\
\hline & & \multicolumn{4}{|c|}{ Fins" } & \multirow{3}{*}{$\begin{array}{c}\text { Body. } \\
\text { cyc. head } \\
\text { (n) }\end{array}$} & & & \\
\hline & & \multirow{2}{*}{$\begin{array}{c}\text { Rank } \\
1 \\
(\mathrm{n}) \\
\end{array}$} & \multirow{2}{*}{$\begin{array}{c}\text { Rank } \\
2 \\
\text { (n) } \\
\end{array}$} & \multirow{2}{*}{$\begin{array}{c}\text { Rank } \\
3 \\
(\mathbf{n}) \\
\end{array}$} & \multirow{2}{*}{$\begin{array}{c}\text { Rank } \\
4 \\
\text { (n) } \\
\end{array}$} & & & & \\
\hline & & & & & & & & Avg. & Range \\
\hline $10 \mathrm{Apr}$ & 8 & 0 & 0 & 0 & 0 & 0 & 00 & 118 & $117.9-117.9$ \\
\hline $18 \mathrm{Apr}$ & 110 & 8 & 1 & 2 & 2 & 0 & 118 & 121 & $119.3-124.3$ \\
\hline I May & 64 & 3 & 1 & 0 & 0 & 0 & 6.3 & 130 & One measurement \\
\hline 2 Mas & 67 & 3 & 0 & 0 & 0 & 0 & 4.5 & 124 & One measurement \\
\hline 7 Mas & 53 & 1 & 1 & 0 & () & 0 & $3 . X$ & 120 & $118.3-121.2$ \\
\hline $15 \mathrm{Mar}$ & 8 & 0 & 0 & 0 & 0 & 0 & 0.0 & 115 & One measurement \\
\hline $16 \mathrm{May}$ & 45 & 0 & 0 & 0 & 0 & 0 & 0.0 & 120 & One measurement \\
\hline 2-Mav. & 69 & 2 & 2 & 1 & () & 0 & 7.2 & 119 & One measurement \\
\hline $23 \mathrm{Mar}$ & 73 & 6 & 3 & 0 & 1 & 0 & 13.7 & 115 & One measuremen \\
\hline $29 \mathrm{Mar}$ & 116 & 3 & 0 & 1 & 2 & 0 & 52 & $12 \mathrm{X}$ & One measurement \\
\hline 5 Jun & 138 & 7 & 2 & 3 & 1 & 1 & 10.1 & 122 & $120.6-123.3$ \\
\hline 12 Jun & 61 & 0 & 0 & 0 & 0 & 0 & 0.0 & 121 & $120.7-121.2$ \\
\hline 20 Jun & 63 & 0 & 0 & 0 & 0 & 2 & 3.2 & 125 & One measurement \\
\hline $3 \mathrm{Jul}$ & 10 & 1 & 0 & 0 & 0 & 0 & 10.0 & 124 & Onc measurement \\
\hline $10 \mathrm{Jul}$ & 89 & () & 0 & 0 & 0 & 0 & 00 & 118 & $116.9-118.7$ \\
\hline $17 \mathrm{Jul}$ & 114 & 2 & 0 & 0 & 1 & 1 & 35 & 121 & $120.4-122.3$ \\
\hline $25 \mathrm{Jul}$ & 60 & 3 & 3 & 0 & 0 & 0 & 100 & 122 & $121.2-121.8$ \\
\hline $31 \mathrm{Jul}$ & 54 & () & 0 & 0 & 0 & 0 & 00 & 117 & $120.4-122.3$ \\
\hline 8 Aug & 57 & 0 & 0 & 0 & 0 & 0 & 0.0 & 114 & One measurement \\
\hline
\end{tabular}

${ }^{a}$ Ramk (determined from percent of total lin area affected with emphysema): $\quad 1=1-5 \%, 2=6-25 \%, 3=26-50 \%$, $4=>50 \%$ 
40 \%. Range TDGS (\%)

GBD \%, Sample

— Daily average TDGS (\%)
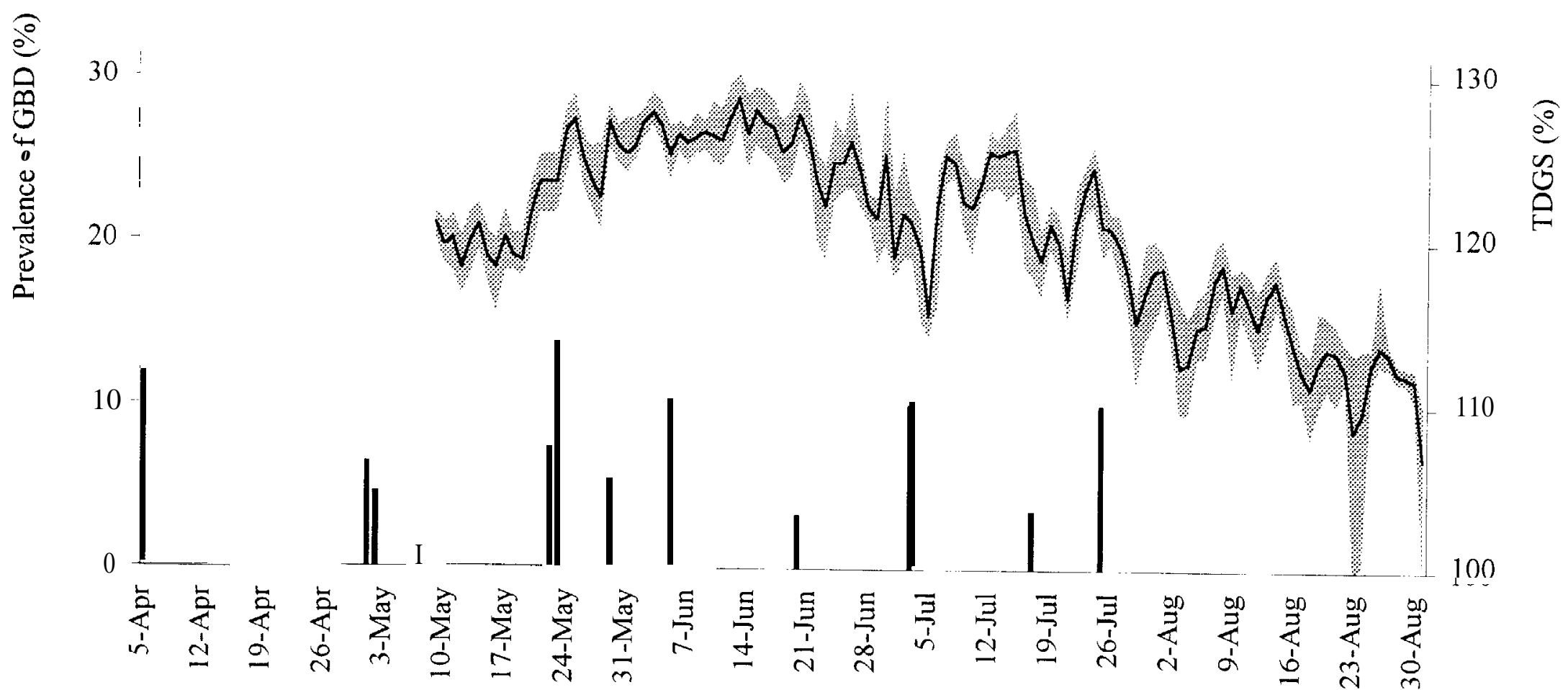

Figure 7. Prevalence of GBD in resident fish collected downstream from Priest Rapids Dam compared with daily average and range of total dissolved gas saturation (TDGS) (COE, Priest Rapids Dam Tailrace). 
Table 7. Numbers sampled, size range, and prevalence of gas bubble disease (GBD) by species for fish collected in Priest Rapids Reservoir, 1996.

\begin{tabular}{|c|c|c|c|c|c|}
\hline \multirow[b]{2}{*}{ Species } & \multirow[b]{2}{*}{ Scientific name } & \multirow{2}{*}{$\begin{array}{c}\text { Sample } \\
\text { (n) }\end{array}$} & \multirow{2}{*}{$\begin{array}{l}\text { Length } \\
\text { rangea } \\
(\mathrm{mm})\end{array}$} & \multicolumn{2}{|c|}{ Prevalence of GBDb } \\
\hline & & & & (n) & $(\%)$ \\
\hline Northern squawfish & Ptychocheilus oregonensis & 651 & $10-545$ & 13 & 2.0 \\
\hline Chiselmouth & Acrocheilus alutaceus & 510 & $41-230$ & 35 & 6.9 \\
\hline Sucker & Catostomus spp. & 290 & $60-595$ & 77 & 266 \\
\hline Redside shiner & Richardsonius balteatus & 248 & $39-160$ & 4 & 1.6 \\
\hline Pumpkinseed & Lepomis gibbosus & 175 & $50-187$ & 9 & 5.1 \\
\hline Peamouth & Mylocheilus caurinus & 99 & $56-295$ & 2 & 20 \\
\hline Sculpin & Cottus spp. & 77 & $66-205$ & 12 & 15.6 \\
\hline Smallmouth bass & Micropterus dolomieui & 70 & $41-400$ & 3 & 4.3 \\
\hline Yellow perch & Perca flavescens & 50 & $43-220$ & 0 & \\
\hline Chinook salmon & Oncorhynchus tshawytscha & 35 & $42-102$ & 0 & \\
\hline Steelhead trout & Oncorhynchus mykiss & 25 & $51-240$ & 0 & \\
\hline Stickleback & Gasterosteus aculeatus & 17 & $31-67$ & 1 & 5.9 \\
\hline Sandroller & Percopsis transmontana & 14 & $83-111$ & 4 & 28.6 \\
\hline Bluegill & Lepomis macrochirus & 6 & $32-113$ & 1 & 16.7 \\
\hline Carp & Cyprinus carpio & 5 & $60-330$ & 0 & \\
\hline Largemouth bass & Micropterus salmoides & 4 & $75-131$ & 0 & \\
\hline American shad & Alosa sapidissima & 1 & 159 & 0 & \\
\hline Lamprey & Lampertra ayresi & 1 & 210 & 0 & \\
\hline Crappie & Pomoxis spp. & 1 & 97 & 0 & \\
\hline Walleve & Stizostedion vitreum & 1 & 272 & 0 & \\
\hline Total salmonids & & 60 & & 0 & 0.0 \\
\hline Total nonsalmonıds & & 2,220 & & 161 & 7.3 \\
\hline
\end{tabular}

\footnotetext{
a Total lengths were measured for all species except salmonids for which fork lengths were measured

b Fiternal examination for signs of GBD using a 2.5- to 5.0-power headband magnifying lens.
} 
Tablc 8. Total dissolved gas saturation (TDGS) at sampling sites. prevalence of external signs of gas bubble disease (GBD) by severity and total prevalence of GBD among resident fish collected in Priest Rapids Resevroir. 1996.

\begin{tabular}{|c|c|c|c|c|c|c|c|c|c|}
\hline \multirow[b]{5}{*}{ Date } & \multirow{5}{*}{$\begin{array}{c}\text { Sample } \\
(\mathrm{n})\end{array}$} & \multicolumn{5}{|c|}{ Preval ence of ( $\mathrm{BBD}$ ) by severity } & \multirow{5}{*}{$\begin{array}{l}\text { Prevalence of } \\
\text { GBD }^{1} \\
(\%)\end{array}$} & \multirow{4}{*}{\multicolumn{2}{|c|}{$\begin{array}{c}\% / 1 \mathrm{TDG} \\
\text { at sampling sites } \\
\end{array}$}} \\
\hline & & \multicolumn{4}{|c|}{ Fins $^{\mathrm{a}}$} & \multirow{4}{*}{$\begin{array}{l}\text { Body, } \\
\text { eye. head } \\
\quad(n)\end{array}$} & & & \\
\hline & & Rank & Rank & Rank & \multirow{3}{*}{$\begin{array}{c}\text { Rank } \\
4 \\
\text { (n) }\end{array}$} & & & & \\
\hline & & 1 & 2 & 3 & & & & & \\
\hline & & (n) & $(\mathrm{n})$ & (n) & & & & Avg. & Range \\
\hline $0 \mathrm{Apr}$ & 48 & 0 & () & 0 & () & 0 & 00 & 122 & $1220-122.0$ \\
\hline $17 \mathrm{Apr}$ & 124 & 1 & 0 & 0 & () & l & 1.6 & 128 & $128.0-128.0$ \\
\hline $30 \mathrm{Apr}$ & 196 & 12 & 3 & 1 & 0 & 3 & 9.7 & 115 & One measurement \\
\hline 8 Mar & 200 & 3 & () & () & 0 & 3 & 30 & 126 & One measurement \\
\hline 13 Mar & 123 & 3 & 2 & 1 & 3 & 0 & 7.3 & 123 & One measurement \\
\hline 20) Mar & 187 & 6 & 3 & () & 2 & & 6.4 & 131 & One measurement \\
\hline $24 \mathrm{Mar}$ & 76 & 2 & 2 & 0 & 1 & 0 & 6.6 & 135 & One measurement \\
\hline $27 \mathrm{Ma}$ & 139 & 17 & 9 & 2 & 1 & 3 & 23.1 & 124 & One measurement \\
\hline $3 \mathrm{Jum}$ & 138 & 15 & 4 & 0 & () & 4 & 16.7 & 133 & One measurement \\
\hline 10.Jun & 176 & 10 & 0 & 0 & 1 & 10 & 12.0 & 130 & One measurement \\
\hline $17 \mathrm{Jun}$ & 118 & 2 & 0 & 0 & 0 & 0 & 1.7 & 109 & One measurement \\
\hline $24 \mathrm{~J} n$ & 71 & 1 & 1 & 1 & 3 & & 9.9 & 21 & $119.0-122.4$ \\
\hline I Jul & 79 & 3 & 1 & 0 & () & 1 & 6.4 & 103 & One measurement \\
\hline $8 . J_{u l}$ & 104 & 0 & O & 0 & 0 & 0 & 00 & 119 & One measurement \\
\hline $15 . J u l$ & 82 & 6 & 2 & 2 & () & 3 & 15.9 & 134 & One measurement \\
\hline $22 . J u l$ & 68 & () & () & () & () & () & 0.0 & 127 & One measurement \\
\hline 29. Jul & 75 & () & 0 & 0 & 0 & 0 & 0.0 & 95 & One measurement \\
\hline 5 Aug & 38 & 0 & 0 & () & () & 0 & 0.0 & 117 & One measurement \\
\hline $12 \mathrm{Aug}$ & 73 & 1 & 0 & 0 & 0 & 0 & 1.4 & 108 & One measurement \\
\hline
\end{tabular}

"Rank (determined from percent of total fin area affected with emphysema): $1=1-5 \%, 2=6-25 \%, 3=26-50 \%$. $4=>50 \%$

${ }^{\mathrm{h}}$ Not including lish with (IBD in lateral line and/or gill. 
\%.:.: Range TDGS (\%)

GBD \%, Sample

40

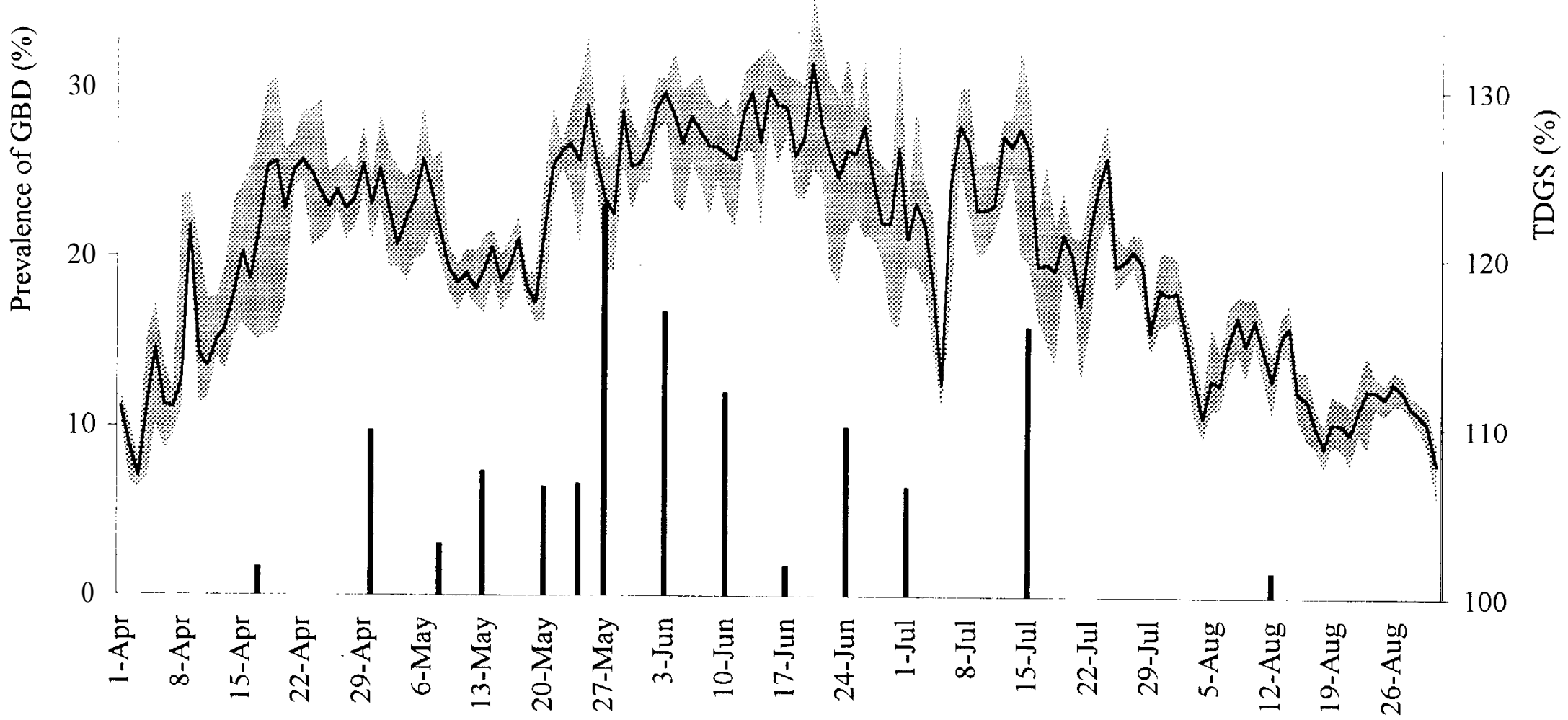

Figure 8. Prevalence of GBD in resident fish collected from the Columbia River in Priest Rapids Reservoir compared with daily average and range of total dissolved gas saturation (TDGS) (COE, Priest Rapids Dam Forebay) 


\section{Downstream from Ice Harbor Dam}

Individuals from 15 of the 17 taxa collected downstream from Ice Harbor Dam displayed external signs of GBD. No salmonids were included, but of the 2,377 nonsalmonids examined $1.2 \%$ exhibited external signs of GBD (Table 9).

From 1 April to 30 April, and 15 May to 24 June, spill at Ice Harbor Dam, along with turbine outages, caused TDGS to reach $142 \%$ downstream from the dam. This period corresponded to the greatest prevalence of external GBD signs in sampled fish Spill volumes up to $116,900 \mathrm{ft}^{3} / \mathrm{sec}$ and $60.9 \%$ of total river flow occurred (Appendix Fig. 4), and daily average TDGS generally remained above $130 \%$ Prevalence of GBD signs within individual daily fish samples was greater than $30 \%$ on 29 April, 30 May, 6 June, 11 June, and 20 June (Table 10)(Fig. 9). Signs of GBD in the lateral line and gill lamellae among fish sampled are summarized in Table 4.

Just outside the mouth of the Snake River lie several small islands in a shallow-water area that is thought to be a rearing area for fall chinook salmon. This area presents the possibility of abnormally high water temperature due to solar heating, and therefore the capacity for increased dissolved gas supersaturation. To evaluate the effects of TDGS on juvenile chinook salmon in this area, we observed several small samples between 19 June and 16 July, when TDGS ranged from 114 to $122 \%$. However, our examination of 22 juvenile chinook salmon revealed no signs of GBD, suggesting that impacts to salmon were minimal. 
Table 9. Numbers sampled, size range, and prevalence of gas bubble disease (GBD) by species for fish collected downstream from Ice Harbor Dam. 1996.

\begin{tabular}{|c|c|c|c|c|c|}
\hline \multirow[b]{2}{*}{ Species } & \multirow[b]{2}{*}{ Scientific name } & \multirow{2}{*}{$\begin{array}{c}\text { Sample } \\
(\mathrm{n})\end{array}$} & \multirow{2}{*}{$\begin{array}{l}\text { Length } \\
\text { range" } \\
(\mathrm{mm})\end{array}$} & \multicolumn{2}{|c|}{$\underline{\text { Prevalence of } \mathrm{GBD}^{\mathrm{h}}}$} \\
\hline & & & & $(\mathrm{n})$ & $(\%)$ \\
\hline Sucker & Catostomuspp. & 422 & 63.595 & 91 & 21.6 \\
\hline Smallmouth bass & Micropterus dolomieui & 392 & $44-535$ & 47 & 12.0 \\
\hline Sculpin & Cottus spp. & 304 & $50-180$ & 40 & 13.2 \\
\hline Largemouth bass & Micropterus salmoides & 202 & $37-526$ & 5 & 2.5 \\
\hline Bluegill & Lepomis macrochirus & 199 & $35-177$ & 4 & 2.0 \\
\hline Northern squawfish & Ptychocheilus oregonensts & 195 & $61-500$ & 24 & 12.3 \\
\hline Pcamouth & Mylocheilus caurinus & 184 & $70-346$ & 14 & 7.6 \\
\hline Yellow perch & Perca flavescens & 163 & $50-436$ & 20 & 12.3 \\
\hline Pumpkmseed & Lepomis gibbosus & 75 & $56-187$ & 6 & 8.0 \\
\hline Chiselmouth & Acrocheilus alutaceus & 71 & $77-306$ & 5 & 7.0 \\
\hline Carp & Cyprinus carplo & 46 & $63-730$ & 5 & 10.9 \\
\hline Crappie & Pomoxis spp. & 38 & $40-276$ & 1 & 2.6 \\
\hline Bullhead & Ictalurus spp. & 33 & $60-368$ & 2 & 6.1 \\
\hline Redside shiner & Richardsonius balteatus & 31 & $53-181$ & 2 & 6.5 \\
\hline Whitefish & Prosopiumspp. & 16 & $100-340$ & 1 & 6.3 \\
\hline American shad & Alosa sapidissima & 4 & $412-440$ & 0 & \\
\hline Channel catfish & Ictalurus punctatus & 1 & 195 & 0 & \\
\hline Unidentified fish & & 1 & -44 & $-\underline{0}$ & \\
\hline Total salmonids & & 0 & & 0 & \\
\hline Total nonsalmonids & & 2.377 & & 267 & 11.2 \\
\hline
\end{tabular}

"Total lengths were measured for all species except salmonids for which fork lengths were measured

"External examination for sighs of GBD using a 2.5- to 5 O-power headband magnifying lens. 
Table 10. Total dissolved gas saturation (TDGS) at sampling sites, prevalence of external signs of gas bubble disease (GBD) by severity, and total prevalence of GBD among resident fish collected downstream from Ice Harbor Dam, 1996.

\begin{tabular}{|c|c|c|c|c|c|c|c|c|c|}
\hline \multirow[b]{4}{*}{$\underline{\text { Datc }}$} & \multirow{4}{*}{$\begin{array}{c}\text { Sample } \\
\text { (n) }\end{array}$} & \multicolumn{5}{|c|}{ Prevalence of GBD by severity } & \multirow{4}{*}{$\begin{array}{c}\text { Prevalence of } \\
\text { GBD }^{\mathrm{b}} \\
(\%)\end{array}$} & \multirow{3}{*}{\multicolumn{2}{|c|}{$\begin{array}{c}\% \text { TDGS } \\
\text { at sampling site/s }\end{array}$}} \\
\hline & & \multicolumn{4}{|c|}{ Fins" } & \multirow{3}{*}{$\begin{array}{c}\text { Body, } \\
\text { eye. head } \\
\text { (n) }\end{array}$} & & & \\
\hline & & \multirow{2}{*}{$\begin{array}{c}\text { Rank } \\
1 \\
(\mathrm{n})\end{array}$} & \multirow{2}{*}{$\begin{array}{c}\text { Rank } \\
2 \\
(\mathrm{n}) \\
\end{array}$} & \multirow{2}{*}{$\begin{array}{c}\text { Rank } \\
3 \\
\text { (n) } \\
\end{array}$} & \multirow{2}{*}{$\begin{array}{c}\text { Rank } \\
4 \\
\text { (n) }\end{array}$} & & & & \\
\hline & & & & & & & & Avg. & Range \\
\hline 16 Арг & 55 & 5 & 6 & 3 & 0 & 1 & 27.3 & 126 & One measurement \\
\hline $24 \mathrm{Apr}$ & 31 & 4 & 0 & 0 & 0 & 1 & 16.1 & 118 & One measurement \\
\hline $29 \mathrm{Apr}$ & 9() & 20 & 8 & 0 & 0 & 6 & 37.8 & 129 & $122.3-135.3$ \\
\hline 6 May & 108 & 3 & 1 & 0 & 0 & 3 & 6.5 & 118 & One measurement \\
\hline 9 Mar & 1 ox & 2 & 1 & 0 & 0 & 5 & 7.7 & 117 & $113.4-120.6$ \\
\hline 14 May & 62 & 0 & 1 & 0 & 0 & 2 & 4.8 & 123 & One measurement \\
\hline 21 Mar & 118 & 12 & 5 & 4 & 0 & 2 & 19.5 & 132 & $125.2-137.8$ \\
\hline 2x Mar & 154 & 15 & 6 & 2 & 0 & 5 & 18.1 & 130 & One measurement \\
\hline 30 Mas & 93 & 4 & 7 & 1 & 4 & 15 & 33.3 & 135 & $131.1-137.9$ \\
\hline 4 Jun & 78 & 6 & 0 & 2 & 1 & 10 & 24.3 & 129 & $124.3-136.6$ \\
\hline 6 Jun & 51 & 6 & 4 & 2 & 1 & 5 & 35.3 & 125 & $113.9-136.0$ \\
\hline $11 \mathrm{Jun}$ & 31 & 6 & 3 & I & 0 & 1 & 35.5 & 116 & $106.9-125.2$ \\
\hline 13 Jun & 189 & 7 & 1 & 0 & 0 & 1 & 4.7 & 123 & One measurement \\
\hline $19 \mathrm{Jun}$ & 78 & 2 & 0 & 0 & 1 & 0 & 3.8 & 120 & $117.1-122.0$ \\
\hline 20 Jun & 34 & 2 & 0 & 1 & 0 & 9 & 35.3 & 120 & One measurement \\
\hline 25 Jun & 70 & 1 & 1 & 0 & 0 & 6 & 11.5 & 114 & $113.9-114.0$ \\
\hline $2 \mathrm{Jul}$ & 63 & 2 & 0 & 0 & 0 & 1 & 4.8 & 121 & One measurement \\
\hline 9 Jul & 93 & 1 & 0 & 0 & 0 & 1 & 2.2 & 116 & $110.0-121.1$ \\
\hline $1 \mathrm{lJul}$ & 57 & 2 & 0 & 0 & 0 & 0 & 3.5 & 119 & $118.2-120.2$ \\
\hline $16 \mathrm{Jul}$ & 19 & 0 & 0 & 0 & 0 & 0 & 0.0 & 115 & One measurement \\
\hline $18 \mathrm{Jul}$ & 71 & 2 & 0 & 0 & 0 & 0 & 2.8 & 103 & One measurement \\
\hline $24 \mathrm{Jul}$ & 79 & 0 & 0 & 0 & 0 & 0 & 0.0 & 103 & $101.1-104.9$ \\
\hline 30 Jul & 88 & 3 & 0 & 0 & 0 & 0 & 3.4 & 100 & One measurement \\
\hline I Aug & 28 & 1 & 0 & 0 & 0 & 0 & 3.6 & 124 & One measurement \\
\hline 6 Aug & 94 & 0 & 0 & 0 & 0 & 0 & 0.0 & 105 & $102.7-106.5$ \\
\hline 7 Aug & 48 & 0 & 0 & 0 & 0 & 0 & 0.0 & 102 & One measurement \\
\hline 13 Aug & 62 & 1 & 0 & 0 & 0 & 0 & 1.6 & 105 & One measurement \\
\hline 15 Aug & 166 & 0 & 0 & 0 & 0 & 1 & 0.0 & 107 & $99.3-113.8$ \\
\hline
\end{tabular}




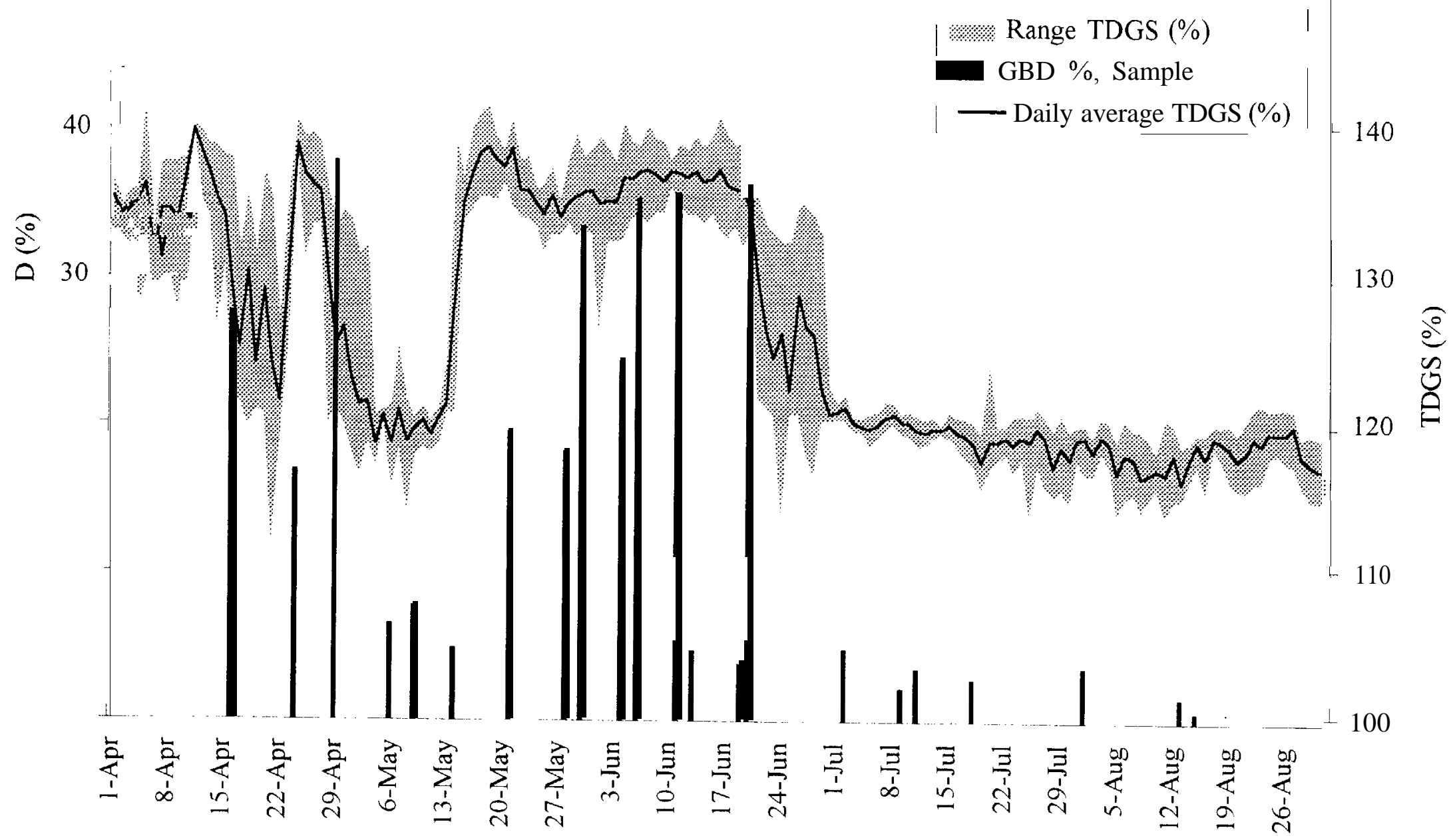

Figure 9. Prevalence of GBD in resident fish collected from the Snake River in Ice Harbor Dam tailrace compared with daily average and range of total dissolved gas saturation (TDGS) (COE, Ice Harbor Dam tailrace). 


\section{Gas Bubble Disease in Captive Fish Groups}

\section{Downstream from Bonneville Dam}

Results of net-pen holding experiments conducted with resident fish downstream from Bonneville Dam are summarized in Table 11. External signs of GBD were observed among surviving resident fish held in the 0 - to 0.5 - $\mathrm{m}$ pen in all 13 4-day holding periods (prevalence range 4.3-100\%) from 17 May through 9 August, when TDGS ranged from 110 to $140 \%$. Fish held in the 0 - to 4-m pen showed external signs of GBD on 7 of the 13 holding periods (prevalence range $0-58.4 \%$ ); prevalence increased during every 4-day holding period conducted from 17 May to 24 June (Fig 10). External signs of GBD among resident fish held in the 2- to 3m pen were observed in only 3 of the 13 holding periods (prevalence range $0-8.3 \%$ ).

Prevalence of GBD signs in the lateral line among surviving fish groups removed from the 0- to 0 5-m pen was not consistently higher than among fish groups removed from the 0- to 4-m or 2-to 3-m pens during the same 4-day holding periods. Prevalence of GBD signs in the lateral line among fish removed from the 0 - to $05-\mathrm{m}, 0-$ to $4-\mathrm{m}$, and 2- to $3-\mathrm{m}$ pens ranged from 0 to $1000 \%, 0$ to $66.7 \%$, and 0 to $66.7 \%$, respectively.

Signs of GBD in the gills were observed among fish groups removed from the 0 - to $0.5-\mathrm{m}$ pen on 6 of the 134 -day holding periods (prevalence range 16.7-50\%). No GBD signs in gills were observed among fish removed from either the 0 - to 4-m or 2- to 3-m pens.

In holding experiments where prevalence of GBD signs among surviving fish increased, that is, when impacts from GBD affected captive fish, mortality among groups held in 0- to 0.5$\mathrm{m}, 0$ - to $4-\mathrm{m}$, and 2- to $3-\mathrm{m}$ pens ranged from 0 to $83 \%, 0$ to $4 \%$, and remained at $0 \%$, respectively (Table 11). 
33

Table 11 Gas bubble disease (CBD). mortality, and total dissolved gas saturation (TDGS) during net-pen experıments holding resident fish downstream from Bonneville Dam, 1996.

\begin{tabular}{|c|c|c|c|c|c|c|c|c|c|c|c|c|c|c|c|c|c|}
\hline \multirow{3}{*}{$\begin{array}{l}\text { Date/ } \\
\text { Conditions }\end{array}$} & \multirow{2}{*}{\multicolumn{2}{|c|}{$\begin{array}{c}\text { Introduction } \\
\text { cxternal } \\
\text { (IBD) }\end{array}$}} & \multicolumn{6}{|c|}{ Survivors Examined ${ }^{h}$} & & & & \multicolumn{6}{|c|}{ Mortalitics Examined } \\
\hline & & & \multicolumn{2}{|c|}{$\begin{array}{l}\text { externale } \\
\text { (ilBI) }\end{array}$} & \multicolumn{2}{|c|}{$\begin{array}{l}\text { LL }^{d} \\
(\mathrm{i} I 3 \mathrm{I})\end{array}$} & \multicolumn{2}{|c|}{$\begin{array}{l}\text { gille }^{\mathrm{C}} \\
\text { (il3I) }\end{array}$} & \multicolumn{2}{|c|}{ Mortality } & \multirow{2}{*}{$\frac{1) \operatorname{com}^{f}}{(n)}$} & \multicolumn{2}{|c|}{$\begin{array}{l}\text { external } \\
\text { (iBD }\end{array}$} & \multicolumn{2}{|c|}{$\begin{array}{l}\text { LIL }^{d} \\
\text { (GBI) }\end{array}$} & \multicolumn{2}{|c|}{$\begin{array}{l}\text { gille }^{\mathrm{e}} \\
\text { GBD }\end{array}$} \\
\hline & $(n)$ & $\%$ & $(n)$ & $(\%)$ & $(n)^{2}$ & $\% / 11$ & $(n)^{g}$ & $0 / 10$ & (n) & $\%$ & & $(n)^{g}$ & $\%$ & $(n)^{g}$ & $(\%)$ & $(n)^{g}$ & $(\%)$ \\
\hline May 13-17 & \multicolumn{17}{|c|}{ TDGS $^{k} 120 \%(118-123 \%)$} \\
\hline surface $(0-0.5 \mathrm{~m})$ & 25 & $\mathbf{0}$ & 22 & 86.4 & 6 & 33.3 & 6 & 16.7 & 1 & 4.3 & 0 & 1 & 0 & 0 & -- & $\mathbf{0}$ & -- \\
\hline $0.4 \mathrm{~m}$ & 52 & 0 & 50 & 8 & 4 & 25 & 3 & 0 & 2 & 3.8 & $\mathbf{0}$ & 2 & 50 & 2 & 0 & $\mathbf{0}$ & -- \\
\hline deep $(2-3 \mathrm{~m})$ & 21 & 0 & 17 & 5.9 & 3 & 33.3 & 3 & 0 & 0 & 0 & 0 & -- & -- & $-\cdot$ & -- & $-\cdot$ & -- \\
\hline May 20-24 & \multicolumn{17}{|c|}{ TDGS $124 \%(117-138 \%)$} \\
\hline surface $(0-0.5 \mathrm{~m})$ & 12 & $\mathbf{0}$ & 5 & 100 & 4 & 100 & 4 & 0 & 7 & 58.3 & $\mathbf{0}$ & 7 & 100 & 7 & 85.7 & 4 & 25 \\
\hline $0-4 \mathrm{~m}$ & 78 & $\mathbf{0}$ & 73 & 20.5 & 3 & 0 & 3 & 0 & 0 & 0 & $\mathbf{0}$ & $\cdots$ & -- & -- & -- & -- & -- \\
\hline deep $(2-3 \mathrm{~m})$ & 12 & 8.3 & 12 & 8.3 & 3 & 33.3 & 3 & 0 & 0 & 0 & $\mathbf{0}$ & -- & -- & - & - & -- & -- \\
\hline May 28-Jun 1 & \multicolumn{17}{|c|}{ TDGS $126 \%(119-140 \%)$} \\
\hline surface $(0-0.5 \mathrm{~m})$ & 11 & 0 & 7 & 100 & 4 & 75 & 4 & 50 & 4 & 36.4 & $\mathbf{0}$ & 4 & 75 & 4 & 100 & 4 & 75 \\
\hline $0-4 \mathrm{~m}$ & 79 & 5.1 & 77 & 58.4 & 3 & 66.7 & 3 & 0 & 1 & 1.3 & 1 & -. & -- & -- & -- & -- & -- \\
\hline deep $(2-3 m)$ & 8 & 0 & 8 & 0 & 3 & 66.7 & 3 & 0 & 0 & 0 & $\mathbf{0}$ & -- & -- & -- & -- & $-\cdot$ & -- \\
\hline Jun 3-7 & \multicolumn{17}{|c|}{ TDGS $124 \%(122-130 \%)$} \\
\hline $\operatorname{surface}(0-0.5 \mathrm{~m})$ & 19 & 5.3 & 10 & 100 & 4 & 100 & 4 & 25 & 9 & 47.4 & $\mathbf{0}$ & 9 & 100 & 8 & 100 & 5 & 60 \\
\hline $0-4 \mathrm{~m}$ & 71 & 19.7 & 69 & 27.5 & 3 & 33.3 & 3 & 0 & 1 & 1.4 & $\mathbf{0}$ & 1 & 100 & 1 & 100 & 1 & 0 \\
\hline deep $(2-3 \mathrm{~m})$ & 17 & 0 & 17 & 0 & 3 & 0 & 3 & 0 & 0 & 0 & 0 & -- & -- & -- & -- & -- & -- \\
\hline Jun 10-14 & \multicolumn{17}{|c|}{ TDGS $127 \%(123-133 \%)$} \\
\hline $\operatorname{surfacc}(0-0.5 \mathrm{~m})$ & 14 & 0 & 11 & 100 & 4 & 50 & 4 & 50 & 3 & 21.4 & $\mathbf{0}$ & 3 & 100 & 3 & 66.7 & 3 & 100 \\
\hline $0-4 \mathrm{~m}$ & 95 & 13.7 & 90 & 40 & 3 & 0 & 3 & 0 & 2 & 2.2 & $\mathbf{0}$ & 2 & 0 & 2 & 50 & 2 & 0 \\
\hline $\operatorname{deep}(2-3 \mathrm{~m})$ & 13 & 0 & 13 & 7.7 & 3 & 33.3 & 3 & 0 & 0 & 0 & $\mathbf{0}$ & -- & -- & -- & -- & -- &.- \\
\hline Jun 17-21 & \multicolumn{17}{|c|}{ TDGS $126 \%(121-138 \%)$} \\
\hline surface $(0-0.5 \mathrm{~m})$ & 16 & 0 & 15 & 86.7 & 4 & 75 & 4 & 25 & 1 & 6.3 & $\mathbf{0}$ & 1 & 100 & 1 & 100 & 1 & 100 \\
\hline $0-4 \mathrm{~m}$ & 95 & 7.4 & 93 & 12.9 & 5 & 40 & 5 & 0 & l & 1.1 & $\mathbf{0}$ & 1 & 0 & 1 & 100 & 1 & 100 \\
\hline $\operatorname{deep}(2-3 \mathrm{~m})$ & 11 & 0 & 11 & 0 & 3 & 33.3 & 3 & 0 & 0 & 0 & $\approx$ & -- & -- & -- & -- & -- & -- \\
\hline Jun 24-28 & \multicolumn{17}{|c|}{ TDGS $123 \%(117-139 \%)$} \\
\hline surface $(0-0.5 \mathrm{~m})$ & 24 & 0 & 4 & 25 & 4 & 25 & 4 & 25 & 19 & 82.6 & $\mathbf{0}$ & 19 & 78.9 & 19 & 52.6 & 13 & 53.8 \\
\hline $0-4 \mathrm{~m}$ & 60 & 0 & 53 & 13.2 & 5 & 20 & 5 & 0 & 2 & 36 & $\mathbf{0}$ & 2 & 50 & 1 & 0 & 1 & 0 \\
\hline $\operatorname{deep}(2-3 \mathrm{~m})$ & 16 & 0 & 11 & 0 & 3 & 0 & 3 & 0 & 0 & 0 & - & - & -- & -- & -- & -. & -- \\
\hline
\end{tabular}


Table 11 Continued.

\begin{tabular}{|c|c|c|c|c|c|c|c|c|c|c|c|c|c|c|c|c|c|}
\hline \multirow{3}{*}{$\begin{array}{l}\text { Date/ } \\
\text { Conditions }\end{array}$} & \multirow{2}{*}{\multicolumn{2}{|c|}{$\begin{array}{l}\text { Introduction } \\
\text { extemal } \\
\text { (iBD) }\end{array}$}} & \multicolumn{6}{|c|}{ Survivors Examined } & & & & \multicolumn{6}{|c|}{ Mortalities Examined } \\
\hline & & & \multicolumn{2}{|c|}{$\begin{array}{l}\text { external } \\
\text { (IBD }\end{array}$} & \multicolumn{2}{|c|}{$\begin{array}{l}\mathrm{LL}^{\mathrm{d}} \\
\mathrm{GHI})\end{array}$} & \multicolumn{2}{|c|}{$\begin{array}{l}\text { gilli }^{\circ} \\
\text { (GI3D) }\end{array}$} & \multicolumn{2}{|c|}{ Mortalits } & \multirow{2}{*}{$\frac{\text { Decom }^{t}}{(\mathrm{n})}$} & \multicolumn{2}{|c|}{$\begin{array}{l}\text { external }^{-} \\
\text {(iBD }\end{array}$} & \multicolumn{2}{|c|}{$\begin{array}{l}\mathrm{LL}^{\mathrm{d}} \\
\mathrm{GIBD}\end{array}$} & \multicolumn{2}{|c|}{$\begin{array}{l}\text { gille }^{\mathrm{e}} \\
\text { GIBD }\end{array}$} \\
\hline & (n) & $(0 / 11)$ & (n) & $(\% / 11)$ & $(n)^{g}$ & $\%$ & $(n)^{g}$ & $\%$ & (n) & $\%$ & & $(n)^{g}$ & $0 / 1$ & $(n)^{g}$ & $(\%)(n$ & $1)^{g}$ & $(1 / 10)$ \\
\hline Jul 1-S & \multicolumn{17}{|c|}{ TDGS $117 \%(114-120 \%)$} \\
\hline surface $(0-0.5 \mathrm{~m})$ & 20 & 0 & 18 & 33.3 & 6 & 0 & 6 & 0 & 2 & 10 & () & 2 & 50 & 1 & 0 & 1 & 0 \\
\hline $0.4 \mathrm{~m}$ & 70 & () & 65 & 0 & 3 & 33.3 & 3 & 0 & 1 & 1.5 & () & 1 & () & 1 & 0 & 0 & -- \\
\hline $\operatorname{deep}(2-3 \mathrm{~m})$ & 15 & 0 & 12 & 0 & 3 & 0 & 3 & 0 & 3 & 20 & 0 & 2 & 0 & 1 & 100 & 0 & - \\
\hline Jul 8-12 & \multicolumn{17}{|c|}{ TDGS $116 \%(110-120 \%)$} \\
\hline surface $(0-0.5 \mathrm{~m})$ & 22 & 0 & 19 & 105 & 5 & 0 & 5 & 0 & 0 & $\mathbf{0}$ & -- & -- & - & -- & -- & -- & -- \\
\hline $0-4 \mathrm{~m}$ & 67 & 0 & 57 & 0 & 2 & 0 & 2 & 0 & 4 & 6.6 & 0 & 4 & $\mathbf{0}$ & 3 & 0 & 2 & 0 \\
\hline $\operatorname{dcep}(2.3 \mathrm{~m})$ & 14 & 0 & 5 & 0 & 3 & 66.7 & 3 & 0 & 8 & 61.5 & () & 8 & $\mathbf{0}$ & 7 & 57.1 & 6 & 0 \\
\hline Jul 15-19 & \multicolumn{17}{|c|}{ TDGS $114 \%(110-119 \%)$} \\
\hline surface $(0-0.5 \mathrm{~m})$ & 30 & 0 & 17 & 5.9 & 4 & 25 & 4 & 0 & 11 & 393 & 5 & 6 & $\mathbf{0}$ & 6 & 0 & 3 & 0 \\
\hline $0-4 \mathrm{~m}$ & 68 & 0 & 41 & 0 & 3 & 0 & 3 & 0 & 11 & 21.2 & 0 & 11 & $\mathbf{0}$ & 10 & 10 & 5 & 0 \\
\hline $\operatorname{detp}(2-3 \mathrm{~m})$ & 29 & 0 & 10 & 0 & 3 & 0 & 3 & () & 11 & 52.4 & 3 & 8 & $\mathbf{0}$ & 7 & 0 & 4 & 0 \\
\hline Jul 22-26 & \multicolumn{17}{|c|}{ TDGS $118 \%(114-122 \%)$} \\
\hline $\operatorname{surfacc}(0-0.5 \mathrm{~m})$ & 43 & 0 & 33 & 9.1 & 5 & 40) & 5 & $\mathbf{0}$ & 8 & 19.5 & 0 & 8 & 0 & 5 & 40 & 2 & 0 \\
\hline $0-4 \mathrm{~m}$ & 79 & 0 & 53 & 0 & 3 & 0 & 3 & 0 & 1 & 1.9 & () & 1 & 0 & 1 & 0 & 0 & 0 \\
\hline $\operatorname{deep}(2-3 m)$ & 35 & 0 & 21 & 0 & 3 & 0 & 3 & 0 & 0 & 0 & -- & -- & -- &.- & -- & -- & -- \\
\hline Jul 29-Aug 2 & \multicolumn{17}{|c|}{ TDGS $116 \%(114-123 \%)$} \\
\hline surface $(0-0.5 \mathrm{~m})$ & 59 & 3.4 & 27 & 14.8 & 4 & 0 & 4 & 0 & 16 & 37.2 & () & 16 & 18.8 & 12 & 25 & 7 & 0 \\
\hline $0-4 \mathrm{~m}$ & 99 & 0 & 71 & 0 & 2 & 0 & 2 & 0 & 1 & 1.4 & () & 1 & 0 & 1 & 100 & 0 & - \\
\hline deep $(2-3 m)$ & 54 & 11.1 & 36 & 0 & 3 & 0 & 3 & 0 & 1 & 2.7 & 0 & 1 & 0 & 0 & -- & 0 & - \\
\hline Aug S-9 & \multicolumn{17}{|c|}{ TDGS $116 \%(110-122 \%)$} \\
\hline surface $(0-0.5 \mathrm{~m})$ & 38 & 2.6 & 23 & 4.3 & 4 & 25 & 4 & 0 & 6 & 20.7 & 0 & 6 & 33.3 & 6 & 16.7 & 1 & 0 \\
\hline $0-4 \mathrm{~m}$ & 51 & 0 & 36 & 0 & 3 & 33.3 & 3 & 0 & 2 & 5.3 & 0 & 2 & 0 & 2 & 0 & 1 & 0 \\
\hline $\operatorname{deep}(2-3 \mathrm{~m})$ & & & 17 & 0 & 3 & 0 & 3 & 0 & 0 & 0 & -- & -- & -- & -- & -- & -- & -- \\
\hline
\end{tabular}

aish placed in holdingnen at beginning of experment

${ }^{\mathrm{e}}$ Signs of CBD in brachial arteries and gill filaments

bive fish removed from pen at end of experiment.

Number of deadfish that were too decomposed to examine for GHBD signs

${ }^{\mathrm{c}}$ External signs of GBD

d Signs of (G13D) in the lateral linc

g Number of fish with GBD

${ }^{\mathrm{h}}$ Average and range of TDGS during holding period (COI: Skamania) 


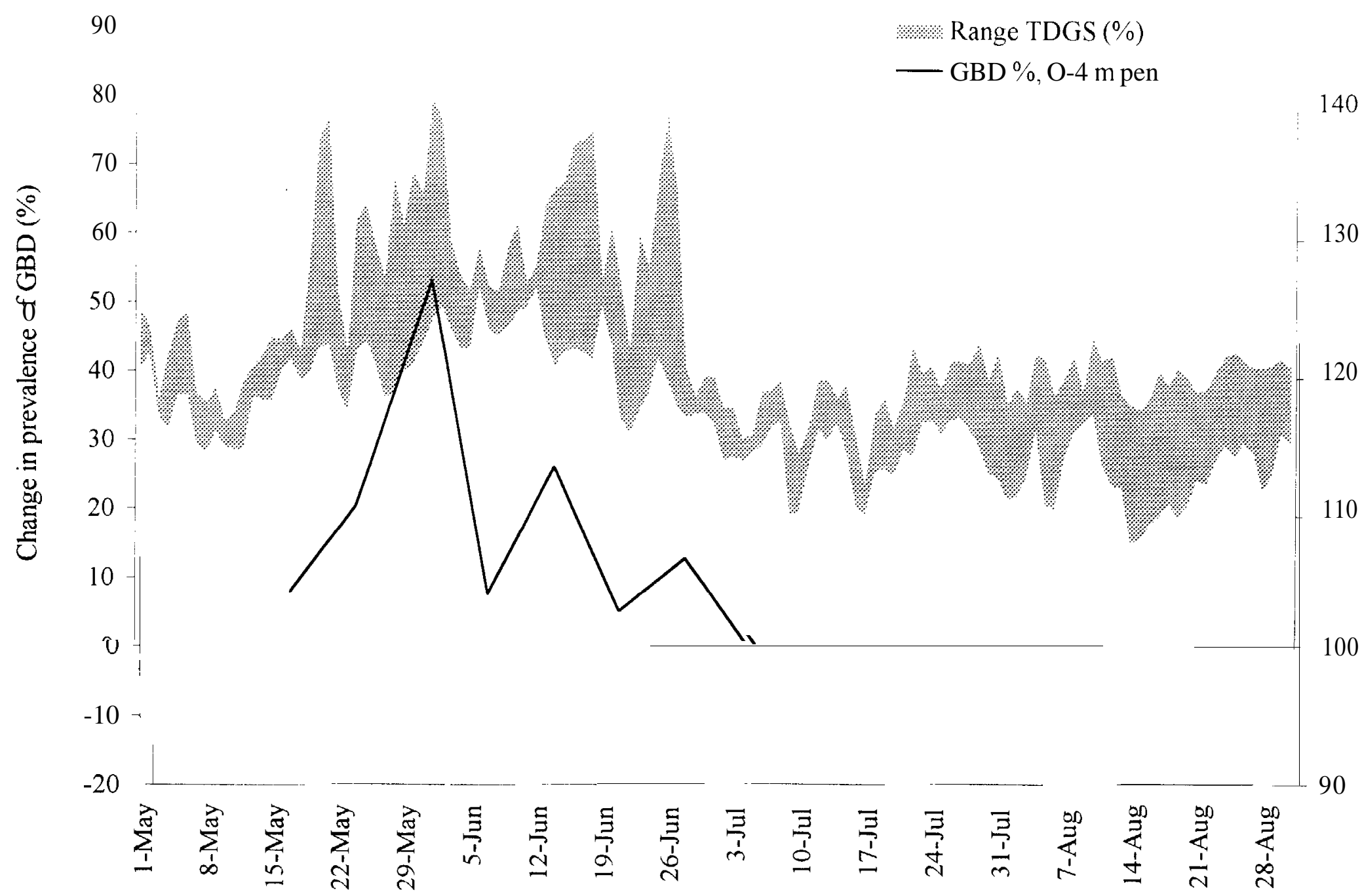

Figure 10. Change in GBD prevalence in resident fish held 4 days in river water downstream from Bonneville Dam compared with range of total dissolved gas saturation (TDGS) (COE, Skamania). 


\section{Priest Rapids Reservoir}

Results of net-pen holding experiments with resident fish conducted upstream from Priest Rapids Dam are summarized in Table 12. External signs of GBD were observed among surviving resident fish held in the 0 - to $0.5-\mathrm{m}$ pen on 15 of the 16 4-day holding periods (prevalence range 20-100\%) from 4 May through 16 August, when TDGS ranged from 111 to $137 \%$. Fish held in the 0 - to 4-m pen showed increases in external signs of GBD on 15 of the 16 holding periods (Fig. 1 1)(prevalence range 0-70\%). External signs of GBD among resident fish held in the 2- to 3-m pen were observed on 6 of the 16 holding periods (prevalence range $0-75 \%$ ).

There was no clear relationship between holding depth and prevalence of GBD signs in the lateral line or gills among surviving fish groups relative to holding depth. Prevalence of GBD signs in the lateral line among surviving fish removed from the 0 - to $0.5-\mathrm{m}, 0$ - to $4-\mathrm{m}$, and 2- to 3$\mathrm{m}$ pens ranged from 0 to $100.0 \%, 0$ to $66.7 \%$, and 0 to $100.0 \%$, respectively. Prevalence of GBD signs in gills ranged from 0 to $75.0 \%, 0$ to $40.0 \%$, and 0 to $66.7 \%$ for fish held in the 0 - to $0.5-\mathrm{m}$, 0- to 4-m, and 2- to 3-m pens, respectively.

In holding experiments where prevalence of GBD signs among surviving fish increased, that is, when GBD impacts affected captive fish, mortality among groups held in 0 - to $0.5-\mathrm{m}, 0$ to $4-\mathrm{m}$, and 2 - to $3-\mathrm{m}$ pens ranged from 0 to $90 \%, 0$ to $33 \%$, and 0 to $56 \%$, respectively (Table 
Table 12 Gas bubble disease (GBD). mortality, and total dissolved gas saturation (TDGS) during net-pen experiments holding resident fish upstream from Pricst Rapids Dam. 1996

\begin{tabular}{|c|c|c|c|c|c|c|c|c|c|c|c|c|c|c|c|c|c|}
\hline \multirow{3}{*}{$\begin{array}{l}\text { Date/ } \\
\text { Conditions }\end{array}$} & \multicolumn{8}{|c|}{ Survivors l samined ${ }^{\mathrm{h}}$} & & & \multirow{3}{*}{$\begin{array}{c}\text { Decom } \\
\text { (n) }\end{array}$} & \multicolumn{6}{|c|}{ Mortalities Examined } \\
\hline & \multicolumn{2}{|c|}{$\begin{array}{c}\text { Introduction } \\
\text { external } \\
\text { GIBD }\end{array}$} & \multicolumn{2}{|c|}{$\begin{array}{l}\text { extemal }^{c} \\
\text { GI3D }\end{array}$} & \multicolumn{2}{|c|}{$\begin{array}{l}\mathrm{LL}^{\mathrm{d}} \\
(\mathrm{iHI})\end{array}$} & \multicolumn{2}{|c|}{$\begin{array}{l}\text { gill }^{e} \\
\text { (IHII) }\end{array}$} & \multicolumn{2}{|c|}{ Mortality } & & \multicolumn{2}{|c|}{$\begin{array}{l}\text { external } \\
\text { (iIBD }\end{array}$} & \multicolumn{2}{|c|}{$\begin{array}{l}\mathrm{LL}^{\mathrm{d}} \\
\text { (iBD) }\end{array}$} & \multicolumn{2}{|c|}{$\begin{array}{l}\text { gille } \\
\text { GBI) }\end{array}$} \\
\hline & $(\mathrm{n})$ & $\%$ & $(n)$ & $0 / 10$ & $(\mathrm{n})$ & $(\%)$ & (n) & $1 / 11$ & (n) & $\% / 11$ & & $(n)$ & $\%$ & $(\mathrm{n})$ & (11) & $(n)$ & $\%$ \\
\hline Apr 30-May 4 & \multicolumn{17}{|c|}{ TDGS $^{k} 123 \%(121-129 \%)$} \\
\hline surface $(0-0.5 \mathrm{~m})$ & 10 & 10 & 9 & 889 & $\mathbf{0}$ & -- & $\mathbf{0}$ & -- & 0 & 0 & 0 & $m$ &.- & -- & -. & $-\cdot$ & -- \\
\hline $0-4 \mathrm{~m}$ & 154 & 9.7 & 149 & 42.3 & $\mathbf{0}$ & -- & $\mathbf{0}$ & - & 10 & 6.3 & 4 & 6 & 16.7 & 5 & 20 & 5 & 0 \\
\hline $\operatorname{deep}(2-3 \mathrm{~m})$ & 9 & 0 & 8 & 0 & $\mathbf{0}$ & -- & 0 & -- & 0 & 0 & 0 & - & -. & - & -- & - & - \\
\hline May 8-12 & \multicolumn{17}{|c|}{ TDGS $119 \%(117-126 \%)$} \\
\hline surface $(0-05 \mathrm{~m})$ & 10 & 10 & 10 & 20 & 5 & G0 & 5 & 0 & $\mathbf{0}$ & $\mathbf{0}$ & -- & - & -- & -- & -- & - & -- \\
\hline $0-4 \mathrm{~m}$ & 114 & 2.9 & 111 & 4.5 & $\mathbf{0}$ & -- & $\mathbf{0}$ & -- & 1 & 0.9 & 0 & 1 & 100 & 1 & 100 & 1 & 100 \\
\hline deep $(2-3 \mathrm{~m})$ & 10 & 20 & 9 & 222 & 5 & 20 & 5 & 0 & 1 & 10 & 1 & -- & -- & - & -- & - & -- \\
\hline May 13-17 & \multicolumn{17}{|c|}{ TDGS $121 \%(119-123 \%)$} \\
\hline surface $(0-0.5 \mathrm{~m})$ & 10 & 0 & 10 & 20 & $\mathbf{0}$ & -- & $\mathbf{0}$ & -- & $\mathbf{0}$ & $\mathbf{0}$ & 0 & $\cdot-$ & $=$ & -- & -- & - & -- \\
\hline $0-4 \mathrm{~m}$ & 93 & 9.7 & 83 & 16.9 & 0 & $-\cdot$ & $\mathbf{0}$ & -. & 3 & 3.5 & $\mathbf{0}$ & 3 & 0 & $\mathbf{0}$ & -- & 0 & -- \\
\hline $\operatorname{deep}(2-3 \mathrm{~m})$ & 10 & 0 & 10 & 0 & 0 & -- & $\mathbf{0}$ & -- & 0 & 0 & 0 & .. & -- & - & -- & - & -- \\
\hline May $20-24$ & \multicolumn{17}{|c|}{ TDGS $127 \%(122-133 \%)$} \\
\hline surface $(0-0.5 \mathrm{~m})$ & 10 & 0 & 10 & 80 & $\mathbf{0}$ & -- & 0 & -- & 0 & $\mathbf{0}$ & 0 & -- &.- & -- & -- & - & -. \\
\hline $0-4 \mathrm{~m}$ & 154 & 7.1 & 134 & 40.3 & 0 & -- & $\mathbf{0}$ & - & 7 & 5 & 1 & 7 & 42.9 & 0 & -- & 0 & -- \\
\hline $\operatorname{deep}(2-3 \mathrm{~m})$ & 10 & 0 & 10 & 0 & $\mathbf{0}$ & - & $\mathbf{0}$ & - & 0 & 0 & 0 & -- & .. &.- & -. & -- & -- \\
\hline May 27-31 & \multicolumn{17}{|c|}{ TDGS $127 \%(124-131 \%)$} \\
\hline surface $(\mathbf{O}-\mathbf{O} .5 \mathrm{~m})$ & 10 & 10 & 5 & 80 & $\mathbf{0}$ & $-\infty$ & $\mathbf{0}$ & -- & 5 & 50 & 0 & 5 & 80 & $\mathbf{0}$ & -- & 0 & -- \\
\hline $0.4 \mathrm{~m}$ & 108 & 26.9 & 97 & 58.8 & $\mathbf{0}$ & -- & $\mathbf{0}$ & - & $\mathbf{0}$ & 0 & 0 & -. & -- & -- & -- & - & - \\
\hline $\operatorname{deep}(2-3 \mathrm{~m})$ & 10 & 20 & 10 & 0 & $\mathbf{0}$ & -- & $\mathbf{0}$ & - & 0 & $\mathbf{0}$ & 0 & $\cdots$ & -- & - & -. &.- & $\ldots$ \\
\hline Jun 3-7 & \multicolumn{17}{|c|}{ TDGS $127 \%(124-131 \%)$} \\
\hline surface $(0-0.5 \mathrm{~m})$ & 10 & 33.3 & 1 & 100 & $\mathbf{0}$ & + & $\mathbf{0}$ & -- & 9 & 90 & 1 & 8 & 100 & 8 & 100 & 8 & 87.5 \\
\hline $0-4 \mathrm{~m}$ & 115 & 21.7 & 67 & 52.2 & $\mathbf{0}$ & -- & $\mathbf{0}$ & .. & 33 & 33 & 0 & 33 & 57.6 & 21 & 38.1 & 21 & 28.6 \\
\hline $\operatorname{deep}(2-3 m)$ & 10 & 10 & 10 & 33.3 & $\mathbf{0}$ & $\cdots$ & $\mathbf{0}$ & -- & 0 & 0 & 0 & $\cdots$ & -- & -- & -. & -- & -- \\
\hline Jun 10-14 & \multicolumn{17}{|c|}{ TDGS $129 \%(123-132 \%)$} \\
\hline surface $(0-0.5 \mathrm{~m})$ & 10 & 20 & 1 & 100 & 1 & 0 & 1 & 0 & 9 & 90 & 0 & 9 & 88.9 & 9 & 100 & 9 & 44.4 \\
\hline $0-4 \mathrm{~m}$ & 144 & 12.5 & 105 & 59 & 5 & 20 & 5 & 20 & 30 & 22.2 & 8 & 22 & 59.1 & 22 & 54.5 & 22 & 13.6 \\
\hline $\operatorname{deep}(2-3 \mathrm{~m})$ & 10 & 10 & 8 & 0 & 5 & 40 & 5 & 0 & 2 & 20 & 0 & 2 & 0 & 2 & 0 & 2 & 0 \\
\hline
\end{tabular}


Table 12. Continued.

\begin{tabular}{|c|c|c|c|c|c|c|c|c|c|c|c|c|c|c|c|c|c|}
\hline \multirow{3}{*}{$\begin{array}{l}\text { Date/ } \\
\text { Conditions }^{2}\end{array}$} & \multicolumn{8}{|c|}{ Survivors Examined" } & & & \multirow{3}{*}{$\begin{array}{c}120 \mathrm{com}{ }^{\mathrm{f}} \\
(\mathrm{n})\end{array}$} & \multicolumn{6}{|c|}{ Mortalities Examined } \\
\hline & \multicolumn{2}{|c|}{$\begin{array}{l}\text { Introduction" } \\
\text { external } \\
\text { (iBI) }\end{array}$} & \multicolumn{2}{|c|}{$\begin{array}{c}\text { cxternal } \\
\text { (iBD) }\end{array}$} & \multicolumn{2}{|c|}{$\begin{array}{l}\text { LI. } \\
\text { (il35) }\end{array}$} & \multicolumn{2}{|c|}{$\begin{array}{l}\text { gill }^{\circ} \\
\text { (ilBI) }\end{array}$} & \multicolumn{2}{|c|}{ Motality } & & \multicolumn{2}{|c|}{$\begin{array}{c}\text { cxternal }^{c} \\
\text { (Gi3I) }\end{array}$} & \multicolumn{2}{|c|}{$\begin{array}{l}\mathrm{LL}^{d} \\
\text { (IBI) }\end{array}$} & \multicolumn{2}{|c|}{$\begin{array}{l}\text { gille } \\
\text { GBI) }\end{array}$} \\
\hline & $(\mathrm{n})$ & $\%$ & $(\mathrm{n})$ & $1 / 10$ & $(\mathrm{n})$ & $(\%)$ & (n) & $\%$ & (n) & $\%$ & & (n) & $1 / 10$ & (n) & $\%$ & (n) & $\%$ \\
\hline Jun 17-21 & \multicolumn{17}{|c|}{ TDGS $128 \%(126137 \%)$} \\
\hline surface $(0-0.5 \mathrm{~m})$ & 10 & 0 & 0 & - & -. & -- & - & -- & 10 & 100 & 3 & 7 & 100 & 7 & 100 & 7 & 57.1 \\
\hline $0-4 \mathrm{~m}$ & 86 & 2.3 & 64 & 59.4 & 5 & 40 & 5 & 40 & 22 & 25.6 & 5 & 17 & 11.8 & 14 & 42.9 & 14 & 0 \\
\hline $\operatorname{deep}(2-3 \mathrm{~m})$ & 10 & 0 & 8 & 0 & 3 & 100 & 3 & 66.7 & 2 & 20 & 0 & 2 & 0 & 1 & 0 & 1 & 100 \\
\hline Jun 24-28 & \multicolumn{17}{|c|}{ TDGS $126 \%(120-132 \%)$} \\
\hline surface $(0-0.5 \mathrm{~m})$ & 10 & 0 & 4 & 50 & $\mathbf{0}$ & -- & 0 & $\ldots$ & 6 & 60 & 1 & 5 & 80 & 4 & 100 & 4 & 75 \\
\hline $0-4 \mathrm{~m}$ & 40 & 18 & 30 & 70 & 0 & -- & 0 & -- & 6 & 16.7 & 0 & 6 & 83.3 & 3 & 333 & 3 & 66.7 \\
\hline deep $(2-3 \mathrm{~m})$ & 10 & 0 & 10 & 0 & $\mathbf{0}$ & -- & 0 & -- & 0 & 0 & 0 & -. & -- & -- & - &.- & -- \\
\hline Jul 1-5 & \multicolumn{17}{|c|}{ TDGS $119 \% \quad(111-129 \%)$} \\
\hline $\operatorname{surface}(0-0.5 \mathrm{~m})$ & 10 & 0 & 8 & 37.5 & 4 & 25 & 4 & 75 & 2 & 20 & 0 & 2 & 100 & 0 & .- & 0 & .- \\
\hline $0-4 \mathrm{~m}$ & 42 & 9.5 & 35 & 17.1 & 5 & 0 & 5 & 0 & 3 & 7.9 & 3 & 0 & -- & 0 & -- & 0 & -- \\
\hline $\operatorname{deep}(2-3 \mathrm{~m})$ & 10 & 0 & 10 & 0 & 5 & 20 & 5 & 0 & 0 & 0 & -. & -- & -- & -- & -- & -. & -- \\
\hline Jul 8-12 & \multicolumn{17}{|c|}{ TDGS $124 \%(120-130 \%)$} \\
\hline $\operatorname{surface}(0-0.5 \mathrm{~m})$ & 10 & 0 & 8 & 87.5 & 4 & 75 & 4 & $\mathbf{5 0}$ & 1 & 11.1 & 0 & 1 & 100 & 0 & -- & 0 & $\cdots$ \\
\hline $0.4 \mathrm{~m}$ & 72 & 0 & 63 & 63.5 & 5 & 20 & 5 & 0 & 4 & 6 & 3 & 1 & 100 & 0 & -. & 0 & - \\
\hline deep $(2-3 \mathrm{~m})$ & 10 & 0 & 10 & 0 & 5 & 20 & 5 & 0 & 0 & 0 & -. & -- & + & -- & -- & -. & -- \\
\hline Jul 15-19 & \multicolumn{17}{|c|}{ TDGS 121\% (114-130\%) } \\
\hline surface $(0-0.5 \mathrm{~m})$ & 10 & 33.3 & 6 & 83.3 & 2 & 100 & 2 & 50 & 4 & 40 & 2 & 2 & 100 & 1 & 100 & 1 & 100 \\
\hline $0-4 \mathrm{~m}$ & 52 & 17.3 & 29 & 31 & 0 & -- & 0 & -- & 14 & 32.6 & 9 & 5 & 20 & 3 & 100 & 3 & 33.3 \\
\hline $\operatorname{deep}(2-3 \mathrm{~m})$ & 10 & 0 & 4 & 75 & 3 & 0 & 3 & 66.7 & 5 & 55.6 & 2 & 3 & 33.3 & 2 & 50 & 2 & 50 \\
\hline Jul 22-26 & \multicolumn{17}{|c|}{ TDGS $122 \%(116-128 \%)$} \\
\hline surface $(0-0.5 \mathrm{~m})$ & 10 & 0 & 9 & 44.4 & 4 & 75 & 4 & 0 & 1 & 10 & 0 & 1 & 100 & 1 & 100 & 1 & 0 \\
\hline $0-4 \mathrm{~m}$ & 43 & 0 & 26 & 11.5 & 5 & 60 & 5 & 40 & 7 & 21.2 & 2 & 5 & 0 & 5 & 20 & 5 & 0 \\
\hline deep $(2-3 \mathrm{~m})$ & 10 & 0 & 9 & 22.2 & 4 & 25 & 4 & 0 & 1 & 10 & 1 & -- & -. & -- & -- & -. & -- \\
\hline Jul 29-Aug 2 & \multicolumn{17}{|c|}{ TDGS $117 \%(116-120 \%)$} \\
\hline surface $(0-0.5 \mathrm{~m})$ & 10 & 0 & 8 & 37.5 & 3 & 33.3 & 3 & 33.3 & 2 & 20 & 2 & -. & .- & -- & .. & $\cdots$ & -- \\
\hline $0-4 \mathrm{~m}$ & 45 & 0 & 34 & 5.9 & 5 & 0 & 5 & 0 & 6 & 15 & 6 & -- & -. & -. & -- & -- & -. \\
\hline $\operatorname{deep}(2-3 \mathrm{~m})$ & 10 & 0 & 10 & 0 & 5 & () & 5 & 0 & 0 & 0 & -- & -- & -- & -- & -. & -- & -- \\
\hline
\end{tabular}


Table 12 Continued

\begin{tabular}{|c|c|c|c|c|c|c|c|c|c|c|c|c|c|c|c|c|c|}
\hline \multirow{3}{*}{$\begin{array}{l}\text { Date/ } \\
\text { Conditions }\end{array}$} & \multicolumn{8}{|c|}{ Survivors Lxamined } & & & \multirow{3}{*}{$\begin{array}{l}\text { Decom. } \\
\text { (n }\end{array}$} & \multicolumn{6}{|c|}{ Mortalities Examined } \\
\hline & \multicolumn{2}{|c|}{$\begin{array}{l}\text { Introduction } \\
\text { external } \\
\text { (GBD }\end{array}$} & \multicolumn{2}{|c|}{$\begin{array}{c}\text { external }^{c} \\
\text { GiBD }\end{array}$} & \multicolumn{2}{|c|}{$\begin{array}{l}\mathrm{LL}^{ل} \\
\mathrm{GBD}\end{array}$} & \multicolumn{2}{|c|}{$\begin{array}{l}\text { gille }^{e} \\
\text { (iI3I) }\end{array}$} & \multicolumn{2}{|c|}{ Mortality } & & \multicolumn{2}{|c|}{$\begin{array}{l}\text { external } \\
\text { (iBD) }\end{array}$} & \multicolumn{2}{|c|}{$\begin{array}{l}\mathrm{LL}^{d} \\
\text { GBD }\end{array}$} & \multicolumn{2}{|c|}{$\begin{array}{l}\text { gill }^{\bullet} \\
\text { GBD }\end{array}$} \\
\hline & $(n)$ & $\%$ & (n) & $\% / 11$ & $(\mathrm{n})$ & $(\%)$ & (11) & $\%$ & (n) & $(\%)$ & & $(n)$ & $\%$ & (n) & $\%$ & $(\mathrm{n})$ & $\%$ \\
\hline 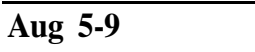 & \multicolumn{17}{|c|}{ TDGS 114\% (111-118\%) } \\
\hline surface $(0-0.5 \mathrm{~m})$ & 10 & 0 & 9 & 55.6 & 4 & 50 & 4 & 25 & 1 & 10 & 0 & 1 & 0 & 1 & 0 & 1 & 0 \\
\hline $0-4 \mathrm{~m}$ & 17 & 0 & 15 & 0 & 3 & 66.7 & 3 & 0 & 1 & 6.3 & 1 & -- & -- & -- & -. & -. & -- \\
\hline $\operatorname{dcep}(2-3 \mathrm{~m})$ & 10 & 0 & 7 & 14.3 & 3 & 66.7 & 3 & 33.3 & 2 & 22.2 & 2 & - & -- & - & $\ldots$ & -- & -- \\
\hline Aug 12-16 & \multicolumn{17}{|c|}{ TDGS 115\% (111-117\%) } \\
\hline $\operatorname{surface}(0-0.5 \mathrm{~m})$ & 10 & 10 & 5 & 40 & 0 & -- & 0 & -- & 5 & 50 & 3 & 2 & 0 & 2 & 50 & 2 & 0 \\
\hline $0-4 \mathrm{~m}$ & 50 & 0 & 38 & 2.6 & 0 & -- & 0 & -- & 9 & 19.1 & 7 & 2 & 0 & 0 & -. & 0 & -- \\
\hline deep_(2-3-m) & 10 & 0 & 4 & 25 & 0 & _- & 0 & _. & 4 & 50 & 2 & 2 & 0 & 2 & 50 & 2 & 50 \\
\hline
\end{tabular}

${ }^{3}$ Fish placed in holding pen at beginning of experiment.

'Signs of GBD in brachial arteries and gill filaments

${ }^{b}$ Live fish removed from pen at end of experiment. $\quad{ }^{\mathrm{f}}$ Number of dead fish that were too decomposed to examine for GBl) signs

'External signs of GBD.

${ }^{d}$ Signs of GBD in the lateral line.

\& Pen depth.

${ }^{\mathrm{h}}$ Average and range of TDGS during holding period (COE, Priest Rapids Dam forebay) 


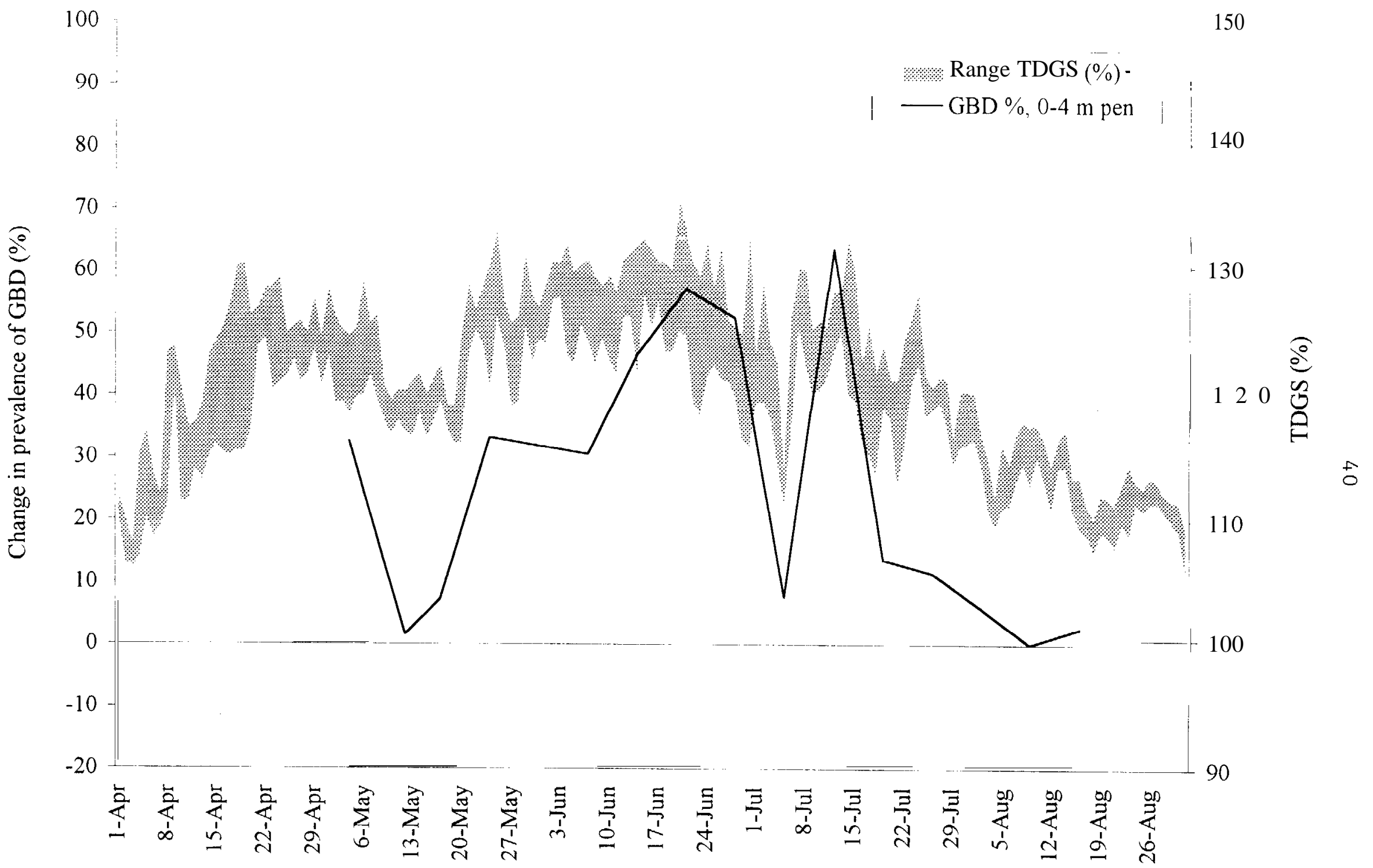

Figure 11. Change in GBD prevalence in resident fish held 4 days in river water in Priest Rapids Reservoir compared with range of total dissolved gas saturation (TDGS) (COE, Priest Rapids Dam forebay). 


\section{Downstream from Ice Harbor Dam}

Results of net-pen holding experiments conducted downstream from Ice Harbor Dam with resident fish are summarized in Table 13. External signs of GBD were observed among surviving resident fish held in the 0 - to 0.5 -m pen in 11 of the 13 -day holding periods (prevalence range 0 $100 \%$ ) from 18 May through 17 August, when TDGS ranged from 114 to 14 1\%. Surviving fish held in the 0- to 4-m pen showed increased external signs of GBD in 9 of the 13 holding periods (Fig. 12)(prevalence range 0-86\%). External signs of GBD among resident fish held in the 2- to 3-m pen were observed in 5 of the 13 holding periods (prevalence range $0-33 \%$ )

Prevalence of GBD signs in the lateral line among surviving fish removed from the 0 - to 0 5-m, 0 - to $4-\mathrm{m}$, and 2- to $3-\mathrm{m}$ pens ranged from 0 to $100.0 \%, 0$ to $66.7 \%$, and 0 to $40.0 \%$, respectively There was no clear relationship between holding depth and prevalence of GBD signs in the gills among surviving fish groups. Signs of GBD in the gills were observed among fish groups removed from the 0 - to $0.5-\mathrm{m}, 0$ - to $4-\mathrm{m}$, and 2- to $3-\mathrm{m}$ pens on 5 , 4 , and 4 of the 154 day holding periods, respectively. Prevalence of GBD signs in the gills ranged from 0 to $80.0 \%, 0$ to $60.0 \%$, and 0 to $40.0 \%$ for fish held in the 0 - to $0.5-\mathrm{m}, 0$ - to $4-\mathrm{m}$, and 2 - to $3-\mathrm{m}$ pens, respectively

In holding experiments where prevalence of GBD signs among surviving fish increased, that is, when GBD affected captive fish, mortality among groups held in 0- to 0.5-m, 0- to 4-m, and 2- to 3-m pens ranged from 0 to $90 \%, 4$ to $40 \%$, and 10 to $22 \%$, respectively (Table 13 ). 


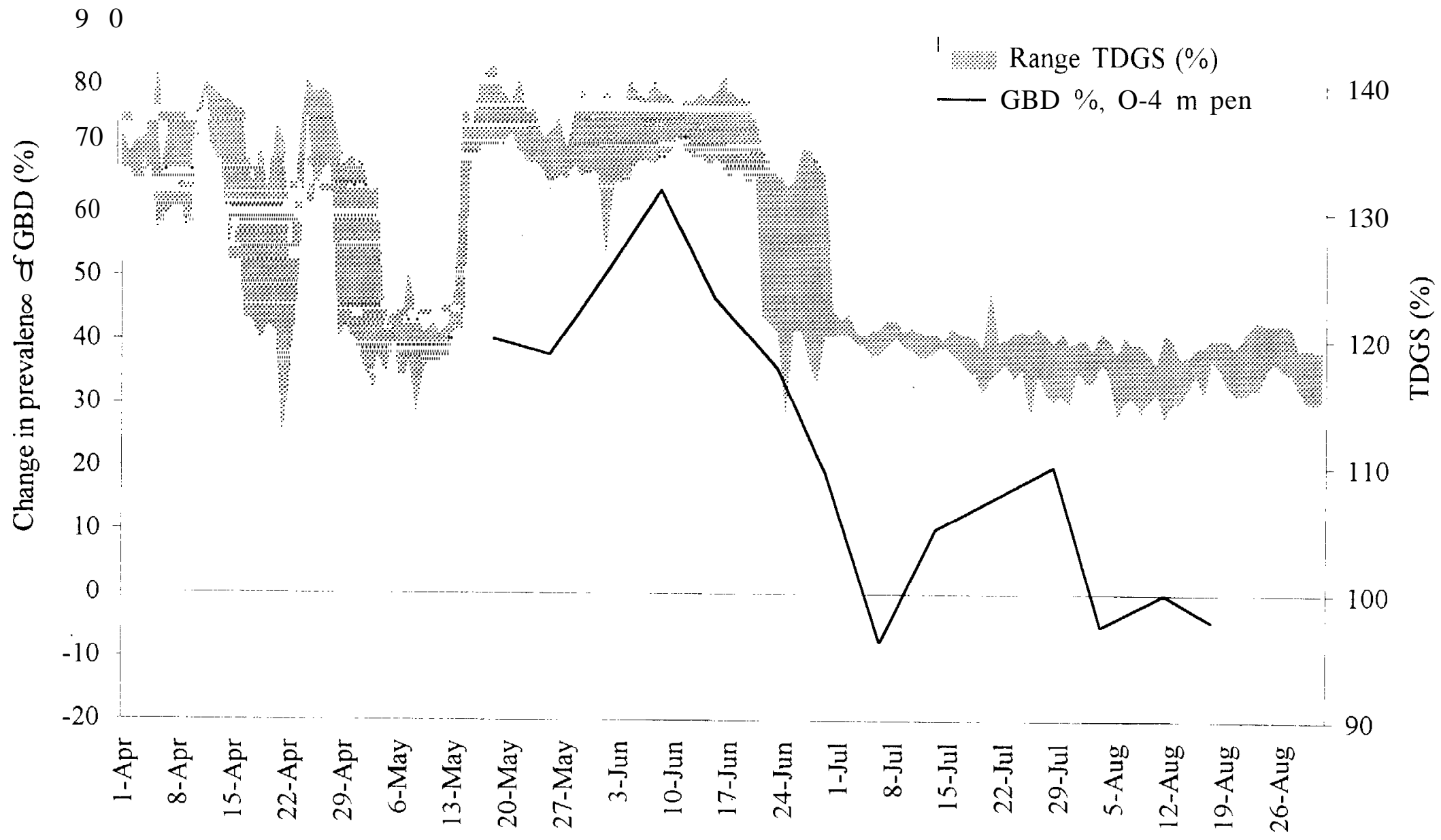

Figure 12. Change in GBD prevalence in resident fish held 4 days in river water downstream from Ice Harbor Dam compared with range of total dissolved gas saturation (TDGS) (COE. Ice Harbor Dam tailrace) 
43

Table 13 Gas bubble discase (GBD). mortality. and total dissolved gas saturation (TDGS) during net-pen experıments holding resident fish downstream from Ice Harbor Dam. 1996.

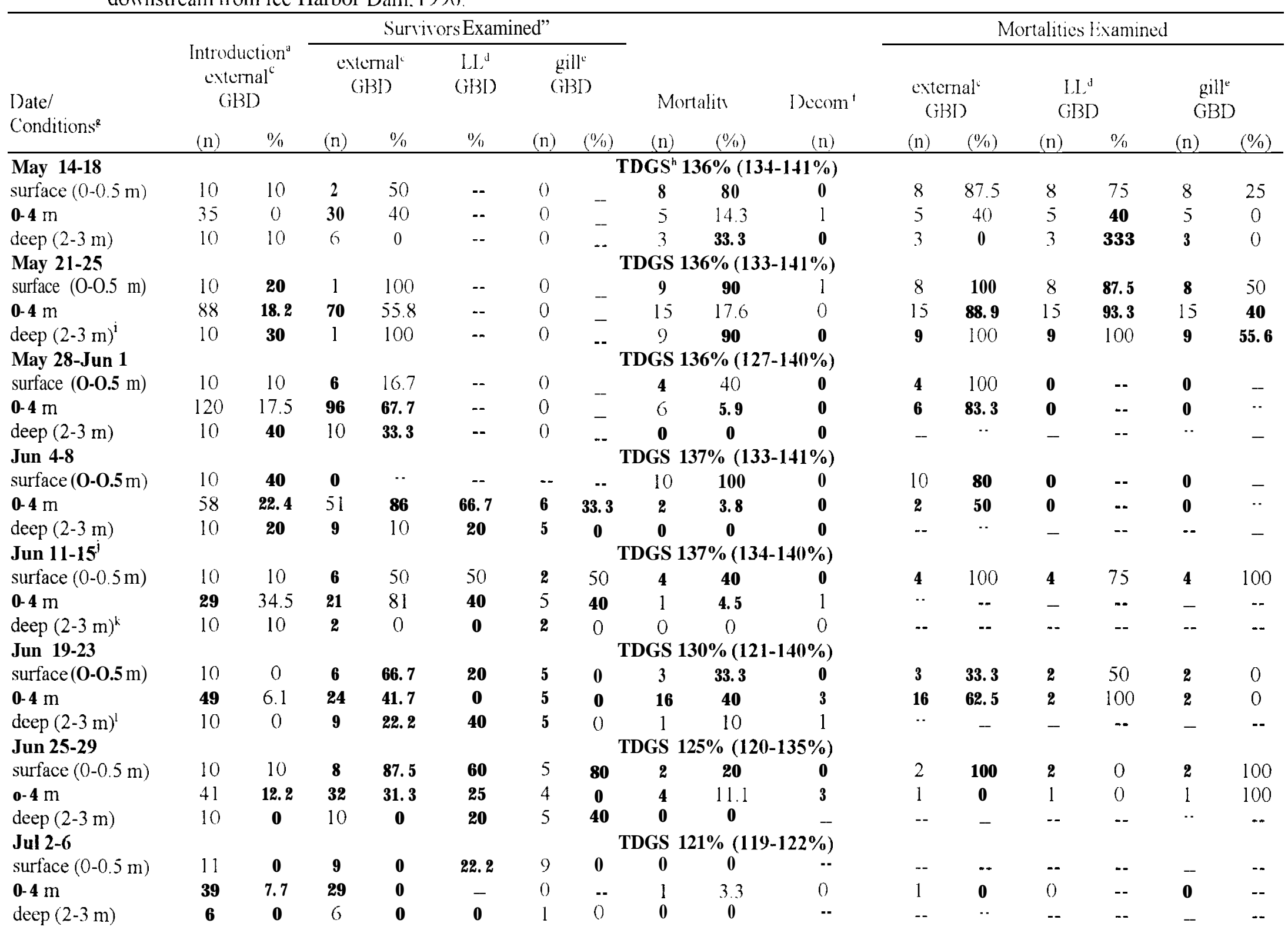


Table 13. Continued.

\begin{tabular}{|c|c|c|c|c|c|c|c|c|c|c|c|c|c|c|c|c|}
\hline \multirow{3}{*}{$\begin{array}{l}\text { Date/ } \\
\text { Conditions }\end{array}$} & \multicolumn{7}{|c|}{ Survivors Examined" } & & & \multirow{3}{*}{$\begin{array}{c}\text { Decom }{ }^{f} \\
(n)\end{array}$} & \multicolumn{6}{|c|}{ Mortalitics Examined } \\
\hline & \multicolumn{2}{|c|}{$\begin{array}{l}\text { Introduction } \\
\text { external } \\
\text { GBD }\end{array}$} & \multicolumn{2}{|c|}{$\begin{array}{l}\text { external" } \\
\text { (iBI) }\end{array}$} & \multirow{2}{*}{$\begin{array}{c}\mathrm{LI}^{\mathrm{d}} \\
\text { (JI3D } \\
0 / 0\end{array}$} & \multicolumn{2}{|c|}{$\begin{array}{l}\text { gill' } \\
\text { (IBD) }\end{array}$} & \multicolumn{2}{|c|}{ Mortality } & & \multicolumn{2}{|c|}{$\begin{array}{l}\text { external } \\
\text { GBD }\end{array}$} & \multicolumn{2}{|c|}{$\begin{array}{l}\mathrm{ILL}^{\mathrm{d}} \\
\mathrm{CHBD}\end{array}$} & \multicolumn{2}{|c|}{$\begin{array}{l}\text { gill’ } \\
\text { GBD }\end{array}$} \\
\hline & $(\mathrm{n})$ & $\% / 11$ & (n) & $\%$ & & (n) & $(\%)$ & (n) & $0 / 11$ & & (n) & $0 / 1$ & $(\mathrm{n})$ & $\%$ & (n) & $\%$ \\
\hline Jul 9-13 & \multicolumn{16}{|c|}{ TDGS $120 \%(119-121 \%)$} \\
\hline surface $(\mathrm{O}-\mathrm{O} .5 \mathrm{~m})$ & 10 & 0 & 8 & 25 & 100 & 4 & S0 & 1 & 11.1 & 0 & 1 & 100 & 1 & 100 & 1 & 100 \\
\hline $\mathrm{O}-4 \mathrm{~m}$ & 67 & 3 & 61 & 13.1 & SO & 6 & 33.3 & 5 & 7.6 & 1 & 4 & 0 & 4 & 0 & 4 & 0 \\
\hline deep $(2-3 \mathrm{~m})$ & 10 & 0 & 6 & 16.7 & 25 & 4 & so & 1 & 14.3 & 0 & 1 & 0 & 1 & 0 & 1 & 0 \\
\hline Jul 16-20 & \multicolumn{16}{|c|}{ TDGS 119\% (116-124\%) } \\
\hline surface $(0-0.5 \mathrm{~m})$ & 10 & 0 & 8 & 12.5 & 0 & 5 & 0 & 2 & 20 & 0 & 2 & 0 & 2 & 0 & 2 & 0 \\
\hline $\mathrm{O}-4 \mathrm{~m}^{\mathrm{m}}$ & 28 & 0 & 4 & 0 & -- & $\mathbf{0}$ & -- & 14 & 77. $\mathrm{x}$ & 9 & 5 & 0 & 1 & 100 & 1 & 0 \\
\hline deep $(2-3 \mathrm{~m})$ & 10 & 0 & 10 & 0 & -- & $\mathbf{0}$ & - & 0 & 0 & - & -- & - & -- & - & -- & -- \\
\hline Jul 24-28 & \multicolumn{16}{|c|}{ TDGS $119 \%(114-121 \%)$} \\
\hline surface $(\mathrm{O}-\mathrm{O} .5 \mathrm{~m})$ & 10 & 0 & 10 & 50 & 60 & 5 & 40 & 0 & 0 & - & -- & - & -- & -- & -- & - \\
\hline $0-4 \mathrm{~m}$ & 56 & 0 & 45 & 20 & 60 & 5 & 40 & 3 & 6.3 & 1 & 2 & 0 & 2 & 0 & 2 & 0 \\
\hline deep (2-3 m) & 10 & 0 & 7 & 14.3 & 33.3 & 3 & 33.3 & 2 & 22.2 & 1 & 1 & 0 & 1 & 0 & 1 & 0 \\
\hline Jul 30-Aug 3 & \multicolumn{16}{|c|}{ TDGS $119 \%(118-120 \%)$} \\
\hline surface $(\mathrm{O}-\mathrm{O} .5 \mathrm{~m})$ & 10 & 0 & 8 & 0 & 0 & 3 & 0 & 0 & 0 & $=$ & -- & - & - & - & -- & - \\
\hline $\mathrm{O}-4 \mathrm{~m}^{\mathrm{n}}$ & 59 & 5.1 & 10 & 0 & -. & 0 & -- & 13 & 56.5 & 3 & 10 & 0 & 0 & -- & 0 & -- \\
\hline deep $(2-3 \mathrm{~m})$ & 10 & 0 & 6 & 0 & 0 & 2 & 0 & 0 & 0 & & -- & -- & -- & - & -- & -- \\
\hline Aug 6-10 & \multicolumn{16}{|c|}{ TDGS $117 \%(114120 \%)$} \\
\hline surface $(\mathrm{O}-\mathrm{O} .5 \mathrm{~m})$ & 10 & 0 & 10 & 20 & 50 & 4 & SO & 0 & 0 & - & -- & - & -- & -- & -- & -- \\
\hline deep $(2-3 \mathrm{~m})$ & 10 & 0 & 8 & 0 & 25 & 4 & 25 & 2 & 20 & 2 & -- & -- & -- & - & -- & - \\
\hline Aug 7-l 1 & \multicolumn{16}{|c|}{ TDGS $117 \%(114-121 \%)$} \\
\hline $\mathrm{O}-4 \mathrm{~m}$ & 38 & 0 & 28 & 0 & -- & $\mathbf{0}$ & -- & 1 & 3.4 & 1 & -- & -- & -- & - & - & .. \\
\hline Aug 13-17 & \multicolumn{16}{|c|}{ TDGS 117\% (115-119\%) } \\
\hline surface $(\mathrm{O}-\mathrm{O} .5 \mathrm{~m})$ & 10 & 0 & 7 & 14.3 & 100 & 1 & $\mathbf{0}$ & 2 & 222 & 1 & 1 & 0 & 1 & 0 & 1 & 0 \\
\hline $0-4 \mathrm{~m}$ & 23 & 4.3 & 19 & 0 & - & $\mathbf{0}$ & $\ldots$ & 4 & 17.4 & 4 & -- & - & -- & - & -- & - \\
\hline deep $(2-3 \mathrm{~m})$ & 8 & $\mathbf{0}$ & 1 & 0 & 0 & 1 & 0 & 0 & 0 & -- & -- &.- & -- &.- & -- & -- \\
\hline $\begin{array}{l}\text { a Fish placed in hol } \\
\text { b Live fish removed } \\
\text { c External signs of } \\
d \text { Signs of GBD in th } \\
\text { e Signs of GBD in b } \\
\text { f Dead fish that wer } \\
g \text { Pen depth. }\end{array}$ & $\begin{array}{l}\text { pen at } \\
\mathrm{m} \text { pen } \\
\text {. } \\
\text { teral li } \\
\text { hial arte } \\
\text { o decon }\end{array}$ & $\begin{array}{l}\text { ginning } \\
\text { nd of } e\end{array}$ & $\begin{array}{l}\text { filam } \\
\text { amin }\end{array}$ & S. & & $\begin{array}{l}\text { "Avel } \\
\text { i Rop } \\
\text { j lish } \\
\text { k'Eigh } \\
\text {, Fish } \\
{ }^{m} \text { Dan } \\
{ }^{n} \text { Sign }\end{array}$ & $\begin{array}{l}\text { ge and } \\
\text { s holdir } \\
\text { yere hel } \\
\text { fish fror } \\
\text { were he } \\
\text { age to } p \\
\text { of man }\end{array}$ & $\begin{array}{l}\text { ige of } \\
\text { pen at } \\
\mathrm{n} \text { dect } \\
\text { the de } \\
\text { in the } \\
\text { preve } \\
\text { al pre }\end{array}$ & $\begin{array}{l}\text { GS du } \\
\text { pth can } \\
\text { id shall } \\
\text { pen pre } \\
\text { ep pen } \\
\text { ed fish } \\
\text { ion we }\end{array}$ & $\begin{array}{l}\text { holding pe } \\
\text { oose during } \\
\text { pens from } \\
\text { nably escap } \\
\text { n } 20 \text {-23 Jun } \\
\text { overy until } \\
\text { bserved. }\end{array}$ & $\begin{array}{l}\mathrm{COF} \\
\text { oldir } \\
\text { Jun } \\
\text { ough } \\
\text { y. Si }\end{array}$ & $\begin{array}{l}\text { I lart } \\
\text { Pen fo } \\
\text { of fo ma }\end{array}$ & $\begin{array}{l}\text { Dam t } \\
\text { Inear } \\
\text { in the } \\
\text { al pro }\end{array}$ & $\begin{array}{l}\text { icc) } \\
\text { ace. }\end{array}$ & & \\
\hline
\end{tabular}




\section{Gas Bubble Disease Effects Model}

We found that mortality in resident fish populations cannot be properly evaluated through sampling because dead fish were rarely observed in the lower Snake and Columbia Rivers; similar conclusions were made by (Merrell et al. 1971) wherein less than 5\% of dead salmon released downstream of Bonneville Dam were observed. The 4-day holding tests in net-pens were intended as a surrogate for mortality evaluations among resident fish, but it appeared that impacts from GBD were greater for captive fish than for free-swimming fish. In 1995 and 1996, downstream from Ice Harbor Dam, average prevalence of external GBD signs for held fish was $13 \%$ greater (range from 25 to $50 \%$ ) than for inriver fish sampled during the previous week. These results suggested that fish held in pens were not a good surrogate for inriver fish in assessment of prevalence of GBD We therefore developed a model for predicting prevalence and severity of external signs of GBD in resident fish in relation to dissolved gas measurements in midriver. We then extrapolated GBD prevalence data to mortality estimates based on a relationship between percent GBD signs and percent mortality derived from our net-pen experiments.

\section{Exposure vs. Gas Bubble Disease Signs}

An exposure index describing effects of increasing, static, and decreasing exposure to total dissolved gas saturation for resident fish was developed by comparing data for external signs of GBD to midriver TDGS data (CROHMS) Few trials with TDGS less than $120 \%$ resulted in fish displaying signs of GBD We speculated that depth distribution of resident fish generally provided sufficient compensation to prevent formation of external signs of GBD. Based on the $120 \%$ threshold, and on statistical trials with shorter and longer exposure periods, we adopted the 
following scale for a 24-hour TDGS daily exposure ranking: $0=<120 \%$,

$1=120-124.9 \%, 2=125-129.9 \%, 3=130-134.9 \%, 4=135-139.9 \%, 5=140-144.9 \%$, and $6=$ $145 \%$ or greater.

Several intervals over which the effects of TDGS were detrimental to resident fish were evaluated, including an index of TDGS exposure over the entire season. However, the narrowest confidence intervals were obtained by using daily ranks, beginning with the sampling day and including the 6 days prior to sampling. These daily ranks were summed to represent a 7-day cumulative exposure index (EI) (Table 14).

We used second-order polynomial regression to compare 7-day exposure index vs. percent GBD (external signs) (Fig. 13). This produced a strong relationship $\left(\mathrm{R}^{2}=079\right)$, leaving us confident that we had developed an EI that could reliably predict external signs of GBD in relation to TDGS exposure $\left[\% \mathrm{GBD}=0.05(\mathrm{EI})^{2} \times 0.21(\mathrm{EI})+0.62\right]$. A bootstraping technique was used to evaluate the statistical analysis, and it produced a nearly identical correlation. The same exposure index and second-order polynomial regression were used to predict external GBD signs of fry in relation to TDGS exposure. Once again this produced a strong relationship $\mathrm{R}^{2}=$ $082\left[\% \mathrm{GBD}=0.050(\mathrm{EI})^{2}+2.83(\mathrm{EI})-0.64\right]$; however, we caution that the fry model is only preliminary There were only 10 samples containing fry (925 total); all were collected below Bonneville Dam in 1996.

Algorithms relating TDGS to percent GBD signs are currently being developed for individual species (smallmouth bass, yellow perch, and peamouth) that display promise for relating percent GBD signs to mortality. Preliminary equations and correlation coefficients for those 
Table 14. Ranking scale and example of the exposure index used to establish impacts of total dissolved gas saturation (TDGS) on resident fish.

\begin{tabular}{|c|c|c|c|c|c|c|}
\hline \multicolumn{2}{|c|}{ Scale } & & \multicolumn{4}{|c|}{ Example } \\
\hline$\%$ TDGS & $\begin{array}{l}\text { Daily } \\
\text { rank }\end{array}$ & & Date & $\% \mathrm{TDGS}^{\mathrm{h}}$ & $\begin{array}{l}\text { Daily } \\
\text { rank }\end{array}$ & $\begin{array}{c}\text { Exposure } \\
\text { index }\end{array}$ \\
\hline $100-119 \%$ & 0 & & Day 6 & 135 & 4 & -- \\
\hline $120-124 \%$ & 1 & & Day 5 & 131 & 3 & -- \\
\hline $125-29 \%$ & 2 & & Day 4 & 124 & 1 & -. \\
\hline $130-134 \%$ & 3 & & Day 3 & 128 & 2 & -- \\
\hline $135-139 \%$ & 4 & & Day 2 & 120 & 1 & -. \\
\hline \multirow[t]{21}{*}{$140-144 \%$} & 5 & & Day 1 & 118 & 0 & -- \\
\hline & & & Day $0^{\mathrm{t}}$ & 122 & 1 & 12 \\
\hline & \multicolumn{5}{|c|}{ Sample Data Below Ice Harbor Dam 1996} & \\
\hline & Date & $\%$ TDGS & $\begin{array}{l}\text { Daily } \\
\text { rank }^{\mathrm{a}}\end{array}$ & $\begin{array}{c}\text { Exposure } \\
\text { index }\end{array}$ & $\begin{array}{c}\text { Daily sample } \\
(\% \mathrm{GBD})^{\mathrm{d}}\end{array}$ & \\
\hline & $23 \mathrm{Apr}$ & 122.0 & 1 & & & \\
\hline & $24 \mathrm{Apr}$ & 138.9 & 4 & & & \\
\hline & $25 \mathrm{Apr}$ & 137.0 & 4 & & & \\
\hline & $26 \mathrm{Apr}$ & 136.2 & 4 & & & \\
\hline & $27 \mathrm{Apr}$ & 135.8 & 4 & & & \\
\hline & $28 \mathrm{Apr}$ & 129.7 & 2 & & & \\
\hline & 29 $\mathrm{Apr}$ & 125.4 & 2 & 21 & $37.8 \%$ & \\
\hline & 30) Apr & 126.5 & 2 & & & \\
\hline & $1 \mathrm{Ma}$ & 123.2 & 1 & & & \\
\hline & 2 May & 121.3 & 1 & & & \\
\hline & 3 May & 121.5 & 1 & & & \\
\hline & 4 May & 118.6 & 0 & & & \\
\hline & $5 \mathrm{May}$ & 120.6 & 1 & & & \\
\hline & $6 \mathrm{Mas}$ & 118.7 & 0 & 6 & $5.5 \%$ & \\
\hline & 7 May & 120.9 & 1 & & & \\
\hline & $8 \mathrm{May}$ & 118.9 & () & & & \\
\hline & 9 May & 119.7 & 0 & 3 & $7.8 \%$ & \\
\hline
\end{tabular}

* Daily rank base on 24-hour mean midriver TDGS (CROHMS)

3 Average daily TD (is near the fish sampling site (CROHMS data).

- Index based on sum of daily ranks for the sampling day and 6-days prior

a Percent of sampled fish displaying external signs of gas bubble disease 
70

- Regression line

-... Confidence interval $(95 \%)$

- - Prediction interval $(95 \%)$

- Daily samples

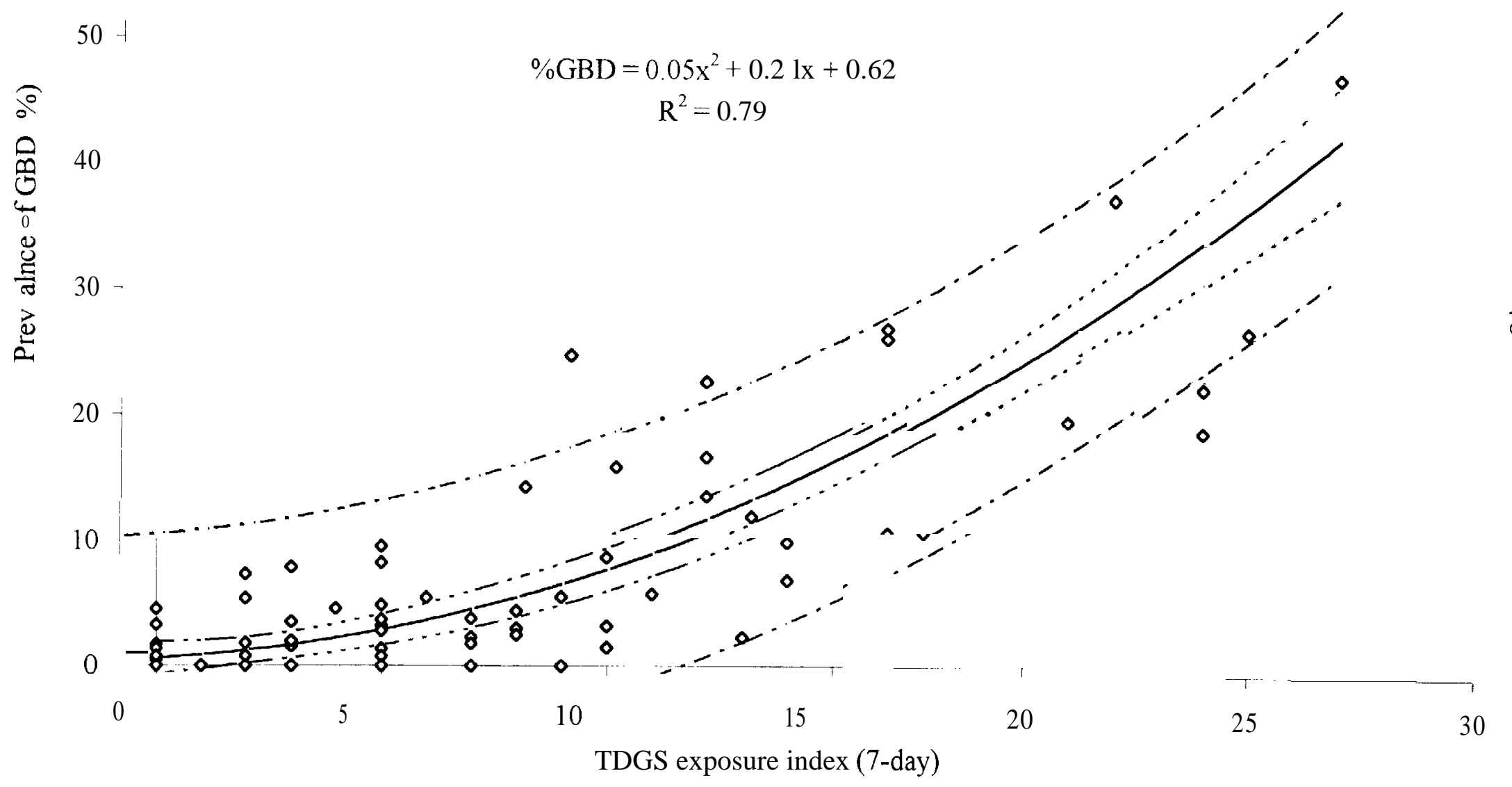

Figure 13. Prevalence of GBD in resident fish collected from the Snake and Columbia rivers compared with 7-day TDGS exposure index (1995-96). 
algorithms were $\mathrm{R}^{2}=0.45\left[\% \mathrm{GBD}=0.0003(\mathrm{EI})^{2}+0.0064(\mathrm{EI})-0.0016\right]$ for smallmouth bass, $\mathrm{R}^{2}=0.68\left[\% \mathrm{GBD}=0.0009(\mathrm{EI})^{2}-0.0062(\mathrm{EI})+0.0065\right]$ for yellow perch, and $\mathrm{R}^{2}=0.36$ $\left[\% \mathrm{GBD}=0.0002(\mathrm{EI})^{2}-0.001(\mathrm{EI})+0.0002\right]$ for peamouth. The individual species models were also developed using small sample sizes, and it should be stressed that these results are preliminary

\section{Gas Bubble Disease Signs vs. Mortality}

In 1995, regression analysis explained $54 \%$ of the observed variability between prevalence of external GBD signs and percent mortality, using all fish species held in net-pens. Although the resulting $\mathrm{R}^{2}$ value $(0.54)$ reflected a significant correlation, we assumed that it was anomalous because the data were distributed at two extremes. When we combined data from 1994, 1995, and 1996 for all fish species, the resulting regression showed no significant relationship. While most fish species did not show a clear relationship between prevalence of GBD signs and percent mortality, due to either small sample size or species-specific behavior, a few species showed promising results

The strongest relationship between prevalence of external GBD signs and percent mortality was for smallmouth bass. $\mathrm{R}^{2}=0.52[\%$ mort $=0.14 \times \log (\%$ GBD $)+0.20]$. However, because of the small sample size and a protracted distribution of data, the relationship was not significant Peamouth and yellow perch showed a trend, but sample sizes were limited. By combining data for the three species, data distributions were improved (Fig. 14). The combined data were evaluated using linear regression and produced the following algorithm: $[\%$ mort $=($ $(2.24 \times \operatorname{sqrt}(G B D \%)-3.51), \mathrm{R}^{2}=0.41$. While this may not explain all of the mortalities, it does at least show some promise. It is not clear whether additional data will show a stronger relationship. 


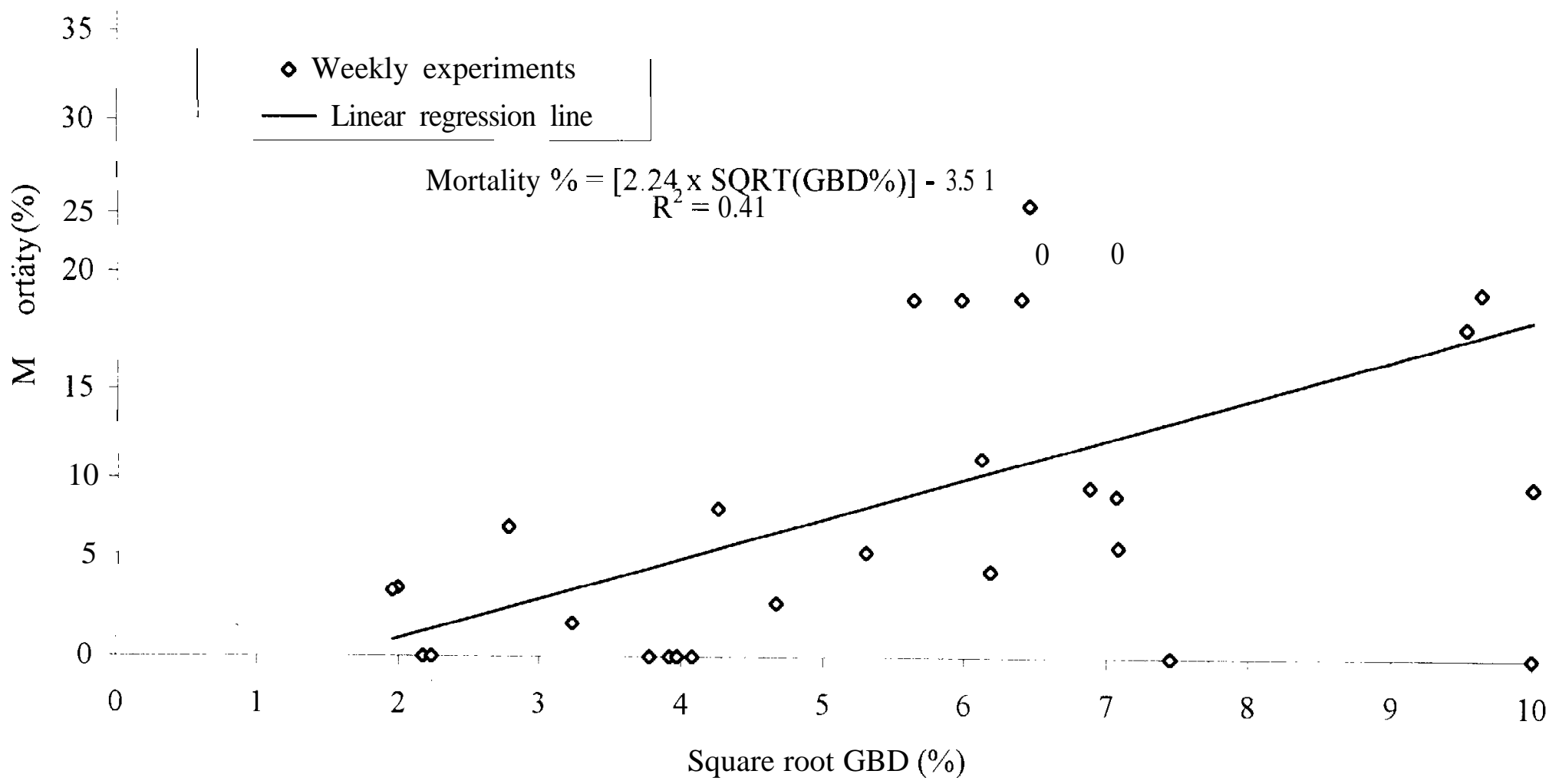

Figure 14. Percent mortality of resident fish held in net-pens in the Snake and Columbia rivers regressed against the square root of percent GBD signs on live fish at the conclusion of each 4-day holding period (1995-96) 


\section{DISCUSSION}

\section{Comparison to 1995 GBD Study}

Downstream from Bonneville Dam in 1995, the daily average TDGS in midriver ranged from 116 to $117 \%$. External signs of GBD were observed on 8 and 29 June, when TDGS peaked at $118 \%$ at the sampling site and $122 \%$ in the river channel (Schrank et al. 1996). In 1996 at these same locations, daily average TDGS in midriver ranged from 111 to $130 \%$. Signs of GBD were observed in resident fish on 28 occasions from 15 March to 12 August. The highest prevalence of GBD occurred during a 10-day period from 3 to 13 June, when up to $16 \%$ of fish sampled displayed external signs of GBD and the daily average TDGS in midriver ranged between 123 and $128 \%$, and TDGS at sampling sites ranged between 121 and $126 \%$.

In Priest Rapids Reservoir during 1995, spill at Wanapum Dam caused high TDGS during May and early June; up to $124.2 \%$ in midriver and $123.7 \%$ at local sampling sites. Prevalence of external signs of GBD was low, ranging from 0 to 5.4\% among resident fish. In 1996, daily average TDGS reached $132 \%$ and was high from mid-April to mid-June. Prevalence of external GBD signs among sampled resident fish ranged from 2 to $23 \%$ through that period.

In 1995 below Ice Harbor Dam, daily average TDGS during peak spill was near 128$130 \%$ from mid-May to mid-June. High prevalence of GBD (11 to 41\%) was observed during this period, but relatively few instances of GBD were observed in the weeks after daily average TDGS had fallen to (and remained at) $118 \%$ or less. In 1996, daily average TDGS during peak spill was near $135 \%$ from mid-May to mid-June. As in 1995, high prevalence of GBD (18 to 
$36 \%$ ) was observed during that period, but prevalence dropped as daily average TDGS fell near or below $120 \%$.

\section{Gas Bubble Disease Effects Model}

We believe that the algorithm relating external GBD signs to TDGS exposure is complete and accurate for fish residing in shallow waters of the Columbia River Basin. However, computed GBD impacts only pertain to those portions of the river where dissolved gas levels are represented by TDGS monitoring data. Areas of lower dissolved gas (by model definition 7\% less) at shoreline peripheries are not properly represented by the TDGS monitoring data. In general, slack water areas cause less risk of GBD to resident fish than the main river

The algorithm relating GBD signs to mortality was not as precise because there appeared to be species-specific behavior that caused high variability for net-pen mortality in multispecies tests Species such as suckers, sculpin, and catfish commonly reside on the bottom, and the environment they came from may have been shallow enough for TDGS to have an impact However, the bottom of our net-pen was 4 meters deep, and therefore provided compensation for TDGS up to $138 \%$ at the surface Other species of fish such as smallmouth bass, yellow perch, and peamouth are not bottom dwellers and were more likely to establish a depth similar to that occupied before they were captured. To evaluate this problem, we split the residents into groups: first by species and then by behaviors While we found no clear relationship for all residents, smallmouth bass, yellow perch, and peamouth showed less variability. We intend to continue tests focusing on these as indicator species. 


\section{CONCLUSIONS AND RECOMMENDATIONS}

1) The algorithm relating GBD signs to TDGS exposure can accurately predict signs in resident fish where continuous TDGS readings are available, therefore we believe the extensive sampling of all species to monitor signs of GBD is no longer necessary Sampling should be continued only on individual species of interest and on a small scale to ensure the accuracy of our model

2) An algorithm relating mortality to GBD signs is not precise, partly due to the effect of combining all sampled species, but separate algorithms by individual species show promise. The holding experiments should be conducted for one more season in areas with consistently high TDGS $(>120 \%$ ), and where sufficient numbers of smallmouth bass, yellow perch, and peamouth can be sampled.

3) We captured fish fry near the water surface; fry that resided deeper in the water column were not targeted. Because of their unusually high position in the water column and their developmental stage, fry are more susceptible to TDGS and were differentiated from mature fish. However, due to limited data, our model relating GBD signs in fry to TDGS should not be relied upon without further sampling and model upgrading. 


\section{ACKNOWLEDGEMENTS}

We thank Lila Charlton, Paul Smith, Craig Zaugg, Michael Cloninger, and Rick De La

Brittonne for their long hours spent in field sampling, often under adverse conditions. We are indebted to Rick Nelson and Dennis Enright for their efforts in construction of equipment and in field sampling. 


\section{REFERENCES}

Bennett, D. 1994. Abundance, habitat, and migration of age 0 fall chinook salmon in the lower Snake River reservoirs. Abstract presented to U.S. Army Corps of Engineers Fish Passage Development and Evaluation Program, I 994 Annual Program Review, (Available from U.S. Army Corps of Engineers, Portland District, P.O. Box 2946 Portland, OR 97208-2946.)

Berggren, T. J., and M. J. Filardo. 1993. An Analysis of Variables Influencing The Migration of Juvenile Salmonids in the Columbia River Basin. N. Am. J Fish. Manage. 13:48-63

Brammer, J A. 199 1. The effects of supersaturation of dissolved gases on aquatic invertebrates of the Bighorn River downstream of Yellowtail Afterbay Dam. Master of Science Thesis, Montana State University, Bozeman, 132 p.

D'Aoust, B G., R. White, and H. Siebold. 1976. An electronic monitor for total dissolved gas pressure. In D. H. Fickeisen and M. J. Schneider (editors), Gas bubble disease, p. 106-110 CONF-74 1033. Technical Information Center, Energy Research and Development Administration, Oak Ridge, TN.

Dawley, E. M. 1986. Effect of 1985-86 levels of dissolved gas on salmonids in the Columbia River. Report to U.S. Army Corps of Engineers, Contract DACW57-85-F-0623, $31 \mathrm{p}$. (Available from Northwest Fisheries Science Center, 2725 Montlake Blvd. E., Seattle, WA 98112.)

Dawley, E M., B. H. Monk, M. H. Schiewe, T. W. Newcomb, F J. Ossiander, and W. J. Ebel. 1976. Effects of long-term exposure to supersaturation of dissolved atmospheric gases on juvenile chinook salmon and steelhead trout in deep and shallow test tanks. $\ln \mathrm{D}$. H. Fickeisen and M. J. Schneider (editors), Gas bubble disease, p. 1- 10. CONF-741033. Technical Information Center, Energy Research and Development Administration, Oak Ridge, TN.

Dell, M. B., M. W. Erho, and B D. Leman. 1974. Occurrence of gas bubble disease symptoms on fish in mid-Columbia River reservoirs. Internal report, Grant County PUD, 49 p. (Available from Public Utility District of Grant County, Ephrata, WA 98823 .)

Ebel, W J 1969. Supersaturation of nitrogen in the Columbia River and its effect on salmon and steelhead trout. U.S. Fish Wildl Serv., Fish Bull. 68: 1-1 1.

Ebel, W. J., and H. L. Raymond. 1976. Effect of atmospheric gas supersaturation on salmon and steelhead trout of the Snake and Columbia Rivers. U.S. Natl. Mar. Fish. Serv., Mar. Fish. Rev. 7:1-14 
Ebel, W. J., H. L. Raymond, G. E. Monan, W. E. Farr, and G. K. Tanonaka. 1975. Effects of atmospheric gas supersaturation caused by dams on salmon and steelhead trout of the Snake and Columbia Rivers Processed Report, 111 p. (Available from Northwest Fisheries Science Center, 2725 Montlake Blvd. E., Seattle, WA 981 12.)

Fidler, L. E., and S B Miller. 1993 British Columbia water quality guidelines for dissolved gas supersaturation Draft report to B.C Ministry of Environment, Canada Department of Fisheries and Oceans, 94 p., plus appendix. (Available from B.C. Ministry of Environment, Water Quality Branch, Water Management Division, 765 Broughton St., Victoria, B.C. V8V1X5)

Merrell, T R Jr , M D Collins, and J W Greenough. 1971. An estimate of mortality of chinook salmon in the Columbia River near Bonneville Dam during the summer run of 1955. U S Fish Wild Serv, Fish Bull 68:3:461-492

Montgomery Watson 1995 Allowable gas supersaturation for fish passing hydroelectric dams. Task 8 - Bubble reabsorption in a simulated smolt bypass system - concept assessment. Report to Bonneville Power Administration, Contract Number DE-AC79-93BP66208 Report number 30090017 p , plus appendixes (Available from Bonneville Power Administration, Portland OR 97208 )

Nebeker, A V , D G Stevens, and J R Brett 1976 Effects of gas supersaturated water on freshwater aquatic invertebrates. In D. H. Fickeisen and M. J. Schneider (editors), Gas bubble disease, p. 5 1-65 CONF-74 1033 Technical Information Center, Energy Research and Development Administration, Oak Ridge, TN.

Schoeneman, D E., R. T Pressey, and C. 0 Junge. 1961 Mortalities of downstream migrant salmon at McNary Dam Trans Am Fish. Soc 90:58-72.

Schrank, B P , B A Ryan, and E. M Dawley. 1996. Evaluation of the effects of dissolved gas supersaturation on fish and invertebrates in Priest Rapids Reservoir, and downstream from Bonneville and Ice Harbor Dams, 1995 Report to the U.S. Army Corps of Engineers, Contract E96940029, (Available from Northwest Fisheries Science Center, 2725 Montlake Blvd. E, Seattle, WA 98 112-2097.)

Stroud, R K and A V Nebeker. 1976. A study of the pathogenesis of gas bubble disease in steelhead trout (Salmo gairdneri) In D H. Fickeisen and M J. Schneider (editors), Gas bubble disease, p 66-7 1 CONF-74 1033 Technical Information Center, Energy Research and Development Administration, Oak Ridge, TN 
Toner, M. A., E. M. Dawley, and B. Ryan. 1995. Evaluation of the effects of dissolved gas supersaturation on fish and invertebrates downstream from Bonneville, Ice Harbor, and Priest Rapids Dams, 1994. Report to the U.S. Army Corps of Engineers, Contract E96940029, $43 \mathrm{p}$ (Available from Northwest Fisheries Science Center, 2725 Montlake Blvd. E., Seattle, WA 98 112-2097)

White, R G., G Phillips, G. Liknes, J Brammer, W Conner, L. Fidler, T Williams, and W Dwyer. 1991 . Effects of supersaturation of dissolved gases on the Fishery of the Bighorn River downstream of the Yellowtail afterbay dam. Report to the U.S. Bureau of Reclamation, 708 p (Available from Montana Cooperative Fishery Research Unit, Montana State University, Bozeman, MN)

Weitkamp, D E and M. Katz. 1980. A review of dissolved gas supersaturation literature Trans Am. Fish. Soc 109:659-702

Wydowski, R S , and R. R. Whitney. 1979. Inland fishes of Washington, University of Washington Press $220 \mathrm{p}$ 


\section{APPENDICES}

Appendix Figure 1

Dam

Appendix Figure 2

Dam

A p pendix Figure 3

Dam

Appendix Figure 4

Dam
1996 flow data, B onneville

.. 1996 flow data, Priest Rapids

1996 f low data, W a n a p u

1996 flow data, Ice Harbor 

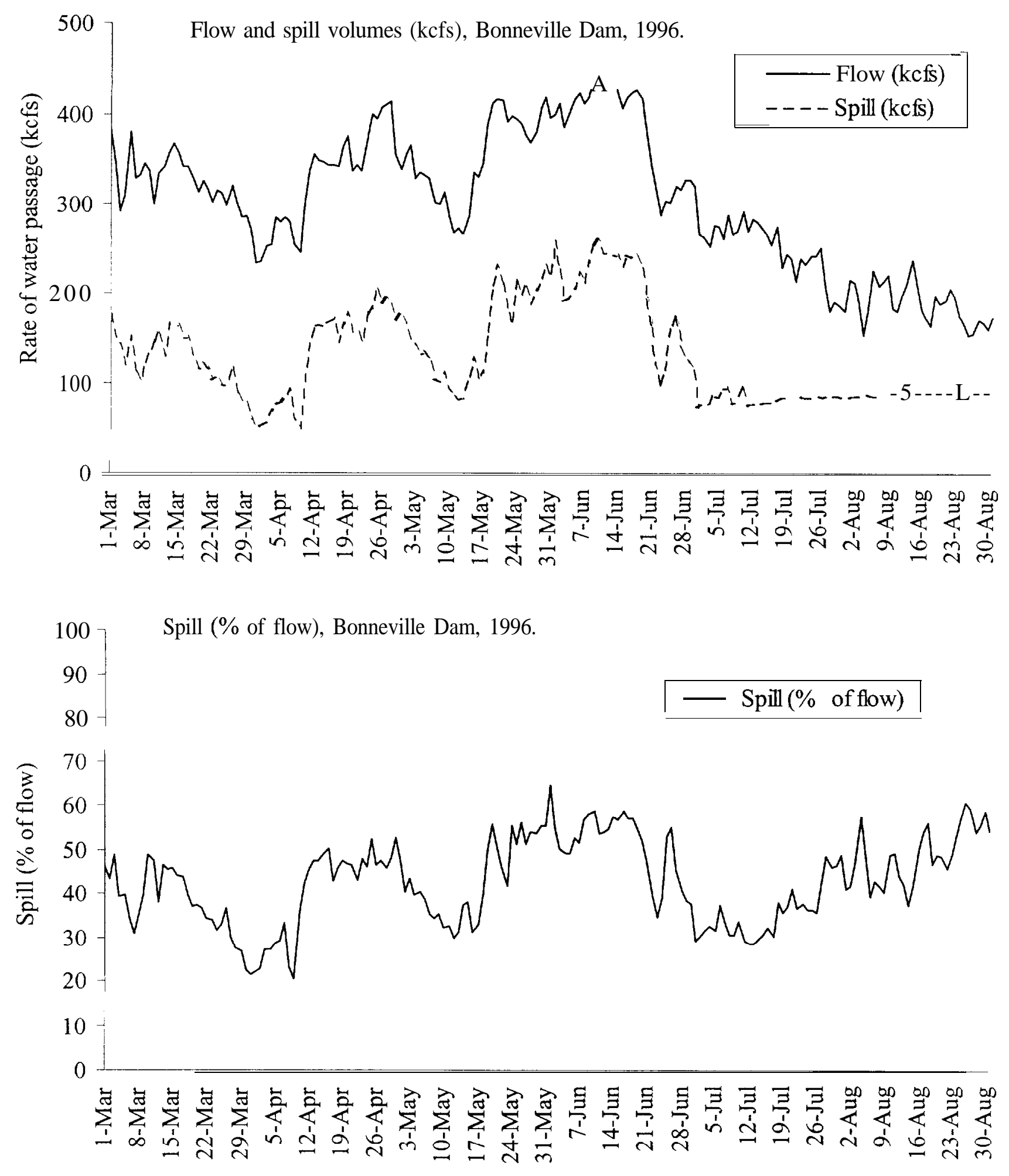

Appendix figure 1. Flow, spill, and \% spill at Bonneville Dam, 1996 (data provided by COE). 

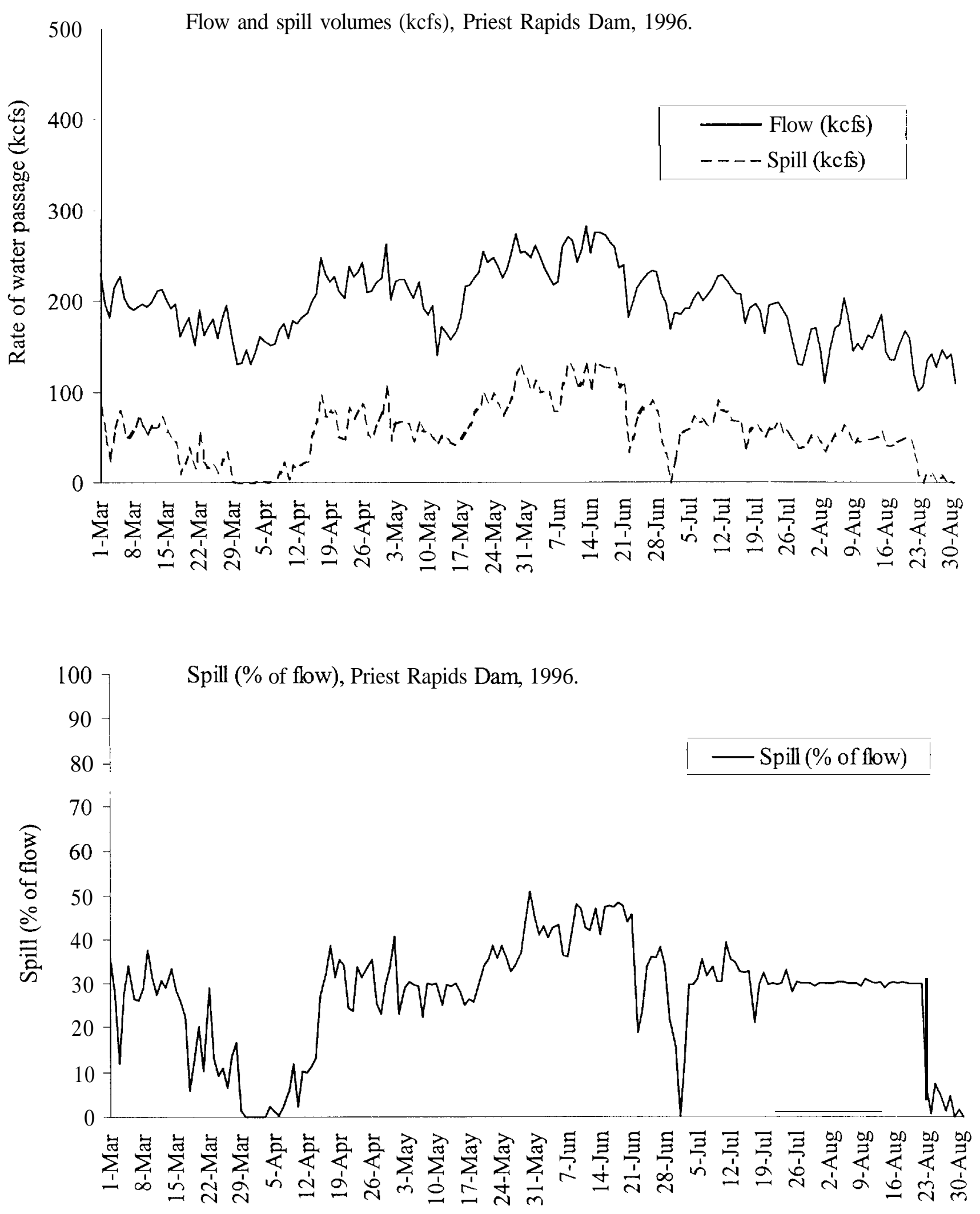

Appendix figure 2. Flow, spill, and \% spill at Priest Rapids Dam, 1996 (data provided by $\mathrm{COE}$ ). 

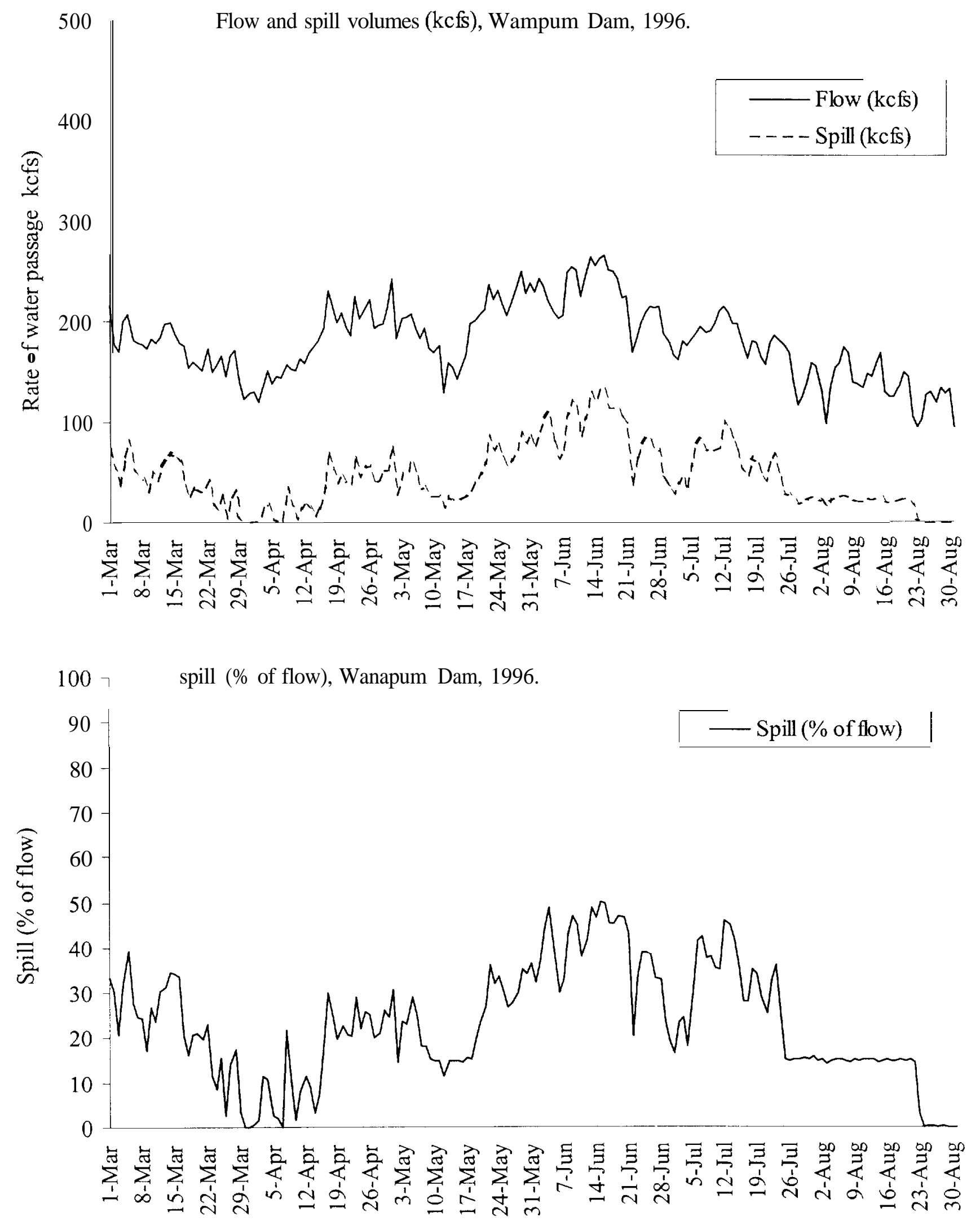

Appendix figure 3. Flow, spill, and \% spill at Wanapum Dam, 1996 (data provided by $\mathrm{COE}$ ). 

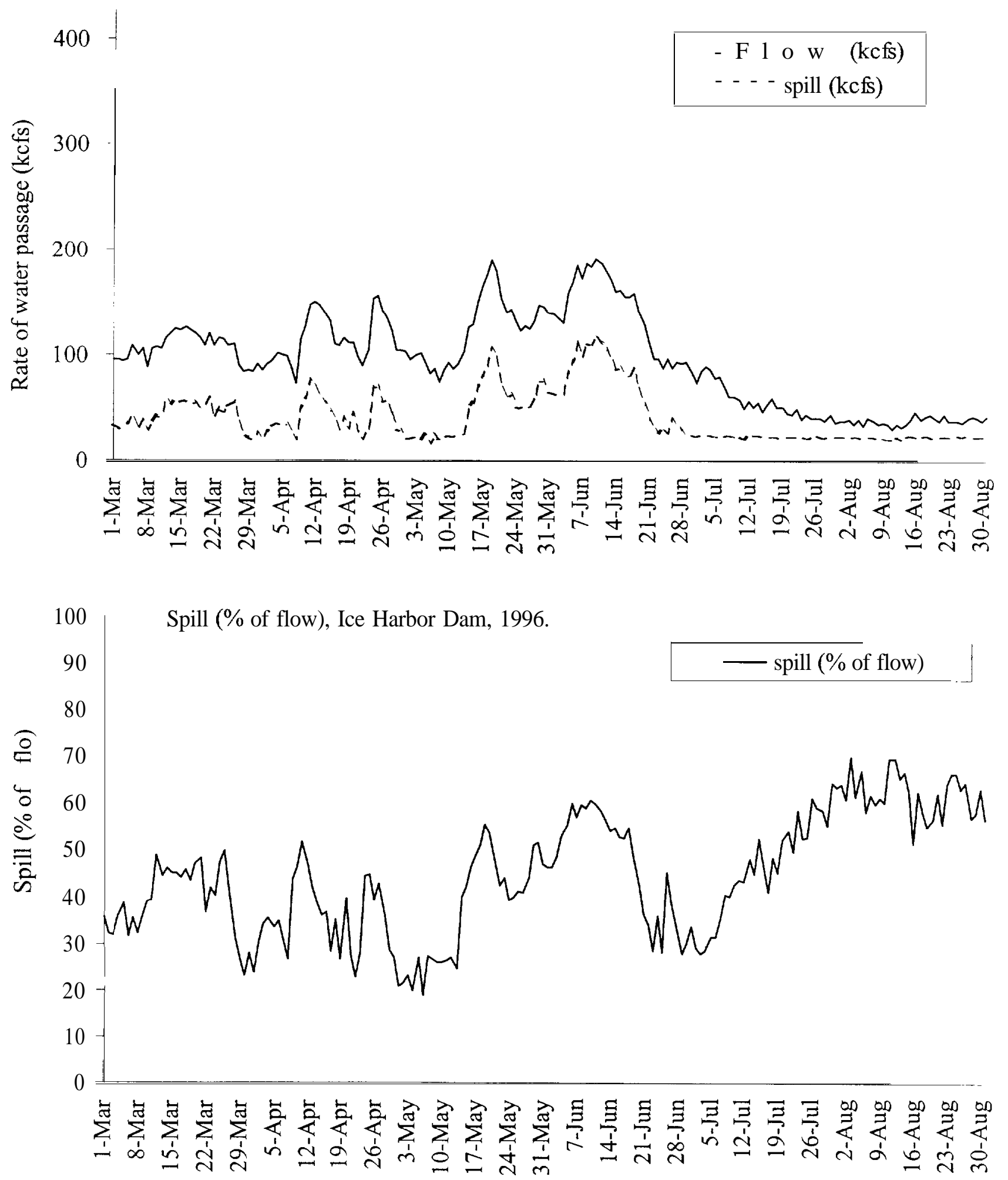

Appendix figure 4. Flow, spill, \% spill at Ice Harbor Darn, 1996

(data provided by $\mathrm{COE}$ ). 